\title{
Skeletal Diversity Through Radical Cyclization of Tetrahydropyridine Scaffolds.
}

Sivaraman Dandapani, Mihai Duduta, James S. Panek*, John A. Porco, Jr*

Department of Chemistry and Center for Chemical Methodology and Library Development (CMLD-BU), Boston University, 590 Commonwealth Avenue, Boston, Massachusetts 02215

\section{Contents}

1. General Experimental Information .................................................................. 2

2. Alkylation of Tetrahydropyridines .............................................................. 2

3. ${ }^{1} \mathrm{H}$ and ${ }^{13} \mathrm{C}$ NMR Spectra of Alkylated Tetrahydropyridines ..................................6

4. Radical Cyclizations and Hydantoin formation ...................................................... 19

5. ${ }^{1} \mathrm{H},{ }^{13} \mathrm{C}$ and 2D NMR Spectra of Products of Radical Cyclizations and

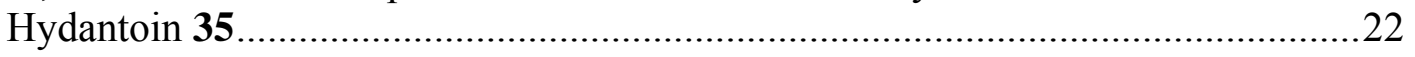

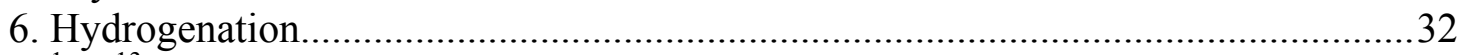

7. ${ }^{1} \mathrm{H},{ }^{13} \mathrm{C}$ and 2D NMR Spectra of Hydrogenated Product 38 ...................................33 
1. General Experimental Information: ${ }^{1} \mathrm{H} N \mathrm{NM}$ and ${ }^{13} \mathrm{C}$ spectra were recorded at 400 $\mathrm{MHz}$ and $100.0 \mathrm{MHz}$ respectively at ambient temperature with $\mathrm{CDCl}_{3}$ as solvent unless otherwise stated. Chemical shifts are reported in parts per million relative to $\mathrm{CDCl}_{3}\left({ }^{1} \mathrm{H}, \delta\right.$ $\left.7.26 ;{ }^{13} \mathrm{C}, \delta 77.0\right)$ or benzene-d6 $\left({ }^{1} \mathrm{H}, \delta 7.15 ;{ }^{13} \mathrm{C}, \delta 128.0\right)$ or acetonitrile-d $3\left({ }^{1} \mathrm{H}, \delta\right.$ 1.94; ${ }^{13} \mathrm{C}, \delta$ 1.24). Data are reported as follows: chemical shift, multiplicity (overlp=overlapping, $\mathrm{s}=$ singlet, $\mathrm{d}=$ doublet, $\mathrm{t}=$ triplet, $\mathrm{q}=$ quartet, $\mathrm{m}=$ multiplet, $\mathrm{br}=$ broad), coupling constant, and integration. All ${ }^{13} \mathrm{C}$ NMR spectra were recorded with complete proton decoupling. High-resolution mass spectra were obtained in the Boston University Chemical Instrumentation Center using a Waters Q-TOF mass spectrometer under chemical ionization. Optical rotations were recorded at $589 \mathrm{~nm}$, and are reported as $[\alpha]_{\mathrm{D}}{ }^{20}$ (concentration in grams $/ 100 \mathrm{~mL}$ solvent). Analytical thin layer chromatography was performed on $0.25 \mathrm{~mm}$ silica gel 60-A plates. Methylene chloride, THF and toluene were purified by passing through two packed columns of neutral alumina.

\section{Alkylation of Tetrahydropyridines}

General Procedure 1 - Enolization and Alkylation of Dienolate from 1: A solution of lithium hexamethyldisilazide in THF $(1.25 \mathrm{M}, 0.16 \mathrm{~mL}, 0.20 \mathrm{mmol})$ was slowly added to a solution of $(2 S, 5 R, 6 R)$-methyl 6-(2-bromophenyl)-2-(but-2-ynyl)-5-methyl-1-(2,2,2trifluoroacetyl)-1,2,5,6-tetrahydropyridi-ne-2-carboxylate 1 (38 $\mathrm{mg}, 0.09 \mathrm{mmol}, 1$ equiv.) and methyliodide (29 $\mu \mathrm{L}, 0.47 \mathrm{mmol}, 5.2$ equiv.) in THF $(0.6 \mathrm{~mL})$ and hexamethylphosphoramide $(0.2 \mathrm{~mL}, 0.9 \mathrm{mmol}, 10$ equiv. $)$ at $-78{ }^{\circ} \mathrm{C}$ and slowly warmed to $0{ }^{\circ} \mathrm{C}$ over $5 \mathrm{~h}$. The reaction was quenched by the addition of saturated brine $(2 \mathrm{~mL})$ and extracted with dichloromethane $(3 \times 15 \mathrm{~mL})$. The combined organic layers were dried with sodium sulafate, filtered and concentrated. Silica gel chromatography with ethyl acetate in hexane (from $0 \%$ to $20 \%$ ) gave the desired product $(2 S, 5 R, 6 R)$-methyl 6-(2bromophenyl)-2,5-dimethyl-1-(2,2,2-trifluoroacetyl)-1,2,5,6-tetrahydropyridine-2carboxylate 15 as colorless oil ( $33 \mathrm{mg}, 85 \%$ yield).

(2S,5R,6R)-Methyl 6-(2-bromophenyl)-2,5-dimethyl-1-(2,2,2-trifluoroacetyl)-1,2,5,6tetrahydropyridine-2-carboxylate $15:{ }^{1} \mathrm{H}$ NMR $(400 \mathrm{MHz}$, $\left.\mathrm{CDCl}_{3}\right) \delta 8.15(\mathrm{dd}, J=7.90,1.6 \mathrm{~Hz}, 1 \mathrm{H}), 7.52(\mathrm{dd}, J=7.9,1.2$
$\mathrm{Hz}, 1 \mathrm{H}), 7.27-7.23(\mathrm{~m}, 1 \mathrm{H}), 7.09(\mathrm{app} . \mathrm{dt}, J=7.7,1.61 \mathrm{~Hz}$,
$1 \mathrm{H}), 5.97(\mathrm{~d}, J=10.2 \mathrm{~Hz}, 1 \mathrm{H}), 5.88(\mathrm{dd}, J=10.2,7.2 \mathrm{~Hz}, 1 \mathrm{H})$, $3 \mathrm{H}), 1.43(\mathrm{~d}, J=7.24 \mathrm{~Hz}, 3 \mathrm{H}) .{ }^{13} \mathrm{C}$ NMR $\left(100 \mathrm{MHz}, \mathrm{CDCl}_{3}\right) \delta 171.5,158.3(\mathrm{q}, J=36.5$ $\mathrm{Hz}), 143.0,132.6,129.0,128.5,128.3,127.9,127.2,121.8,116.0$ (q, $J=287.4 \mathrm{~Hz}), 61.0$ $(\mathrm{q}, J=3.4 \mathrm{~Hz}), 63.0,53.0,36.0,26.4,20.7$. HRMS $\left(\mathrm{CI} / \mathrm{NH}_{3}\right) \mathrm{m} / \mathrm{z}$ calculated for $\left[\mathrm{C}_{17} \mathrm{H}_{17} \mathrm{NO}_{3} \mathrm{~F}_{3} \mathrm{Br}+\mathrm{Na}\right]$ 442.0242, found 442.0260. IR (thin film) $v_{\max } 2956,1746,1694$, $1266,1146 \mathrm{~cm}^{-1} \cdot[\alpha]_{\mathrm{D}}^{20}+32.7^{\circ}\left(\mathrm{c}=1.0, \mathrm{CHCl}_{3}\right)$. 
$(2 S, 5 R, 6 R)-$ Methyl

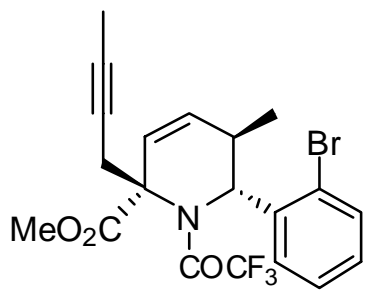

6-(2-bromophenyl)-2-(but-2-ynyl)-5-methyl-1-(2,2,2-trifluoroacetyl)-1,2,5,6-tetrahydropyridine-2-carboxylate 16: This compound was synthesized by general procedure 1 and isolated in pure form as a colorless oil. ${ }^{1} \mathrm{H}$ NMR $\left(400 \mathrm{MHz}, \mathrm{CDCl}_{3}\right) \delta$ $8.03(\mathrm{dd}, J=7.9,1.6 \mathrm{~Hz}, 1 \mathrm{H}), 7.54(\mathrm{dd}, J=7.9,1.2 \mathrm{~Hz}, 1 \mathrm{H})$, 7.27-7.23 (m, 1H), 7.12-7.08 (m, 1H), 6.04-5.92 (m, 2H), 5.50 $(\mathrm{d}, J=1.9 \mathrm{~Hz}, 1 \mathrm{H}), 3.84(\mathrm{~s}, 3 \mathrm{H}), 3.31(\mathrm{qd}, J=16.9,2.6, \mathrm{~Hz}$, 1H) 3.20 (qd, $J=16.9,2.5 \mathrm{~Hz}, 1 \mathrm{H}$ ), 2.57 (app. dp, $J=7.2,1.9$

$\mathrm{Hz}, 1 \mathrm{H}), 1.79(\mathrm{t}, J=2.6 \mathrm{~Hz}, 3 \mathrm{H}), 1.49(\mathrm{~d}, J=7.3 \mathrm{~Hz}, 3 \mathrm{H}) .{ }^{13} \mathrm{C} \mathrm{NMR}\left(100 \mathrm{MHz}, \mathrm{CDCl}_{3}\right)$ $\delta 170.8,159.2(\mathrm{~d}, J=36.9 \mathrm{~Hz}), 143.1,132.8,130.0,128.6,128.2,127.4,126.6,122.5$, $116.0(\mathrm{q}, J=286.4 \mathrm{~Hz}$ ), 79.9, 74.2, 64.8, 61.3, 61.2, 53.1, 36.7, 29.5, 21.3, 3.6. HRMS $\left(\mathrm{Cl} / \mathrm{NH}_{3}\right) \mathrm{m} / \mathrm{z}$ calculated for $\left[\mathrm{C}_{20} \mathrm{H}_{19} \mathrm{NO}_{3} \mathrm{BrF}_{3}+\mathrm{Na}\right] 480.0398$, found 480.0413 . IR (thin film) $v_{\max } 1745,1691,1205,1152 \mathrm{~cm}^{-1} .[\alpha]_{\mathrm{D}}^{20}+5.5^{\circ}\left(\mathrm{c}=0.3, \mathrm{CHCl}_{3}\right)$.

(2S,5R,6R)-Methyl 6-(2-bromophenyl)-5-methyl-2-(prop-2-ynyl)-1-(2,2,2-trifluoroacetyl)-1,2,5,6-tetrahydropyridine-2-carboxylate 17: This compound was synthesized by general procedure 1 and isolated in pure form as a colorless oil. ${ }^{1} \mathrm{H}$ NMR $\left(400 \mathrm{MHz}, \mathrm{CDCl}_{3}\right) \delta$ $8.09(\mathrm{dd}, J=7.9,1.5 \mathrm{~Hz}, 1 \mathrm{H}), 7.28-7.24(\mathrm{~m}, 1 \mathrm{H}), 7.54(\mathrm{dd}, J=$ 8.0, $1.1 \mathrm{~Hz}, 1 \mathrm{H}$ ), 7.10 (app. dt, $J=7.7,1.6 \mathrm{~Hz}, 1 \mathrm{H}$ ), 6.08-6.01 $(\mathrm{m}, 2 \mathrm{H}), 5.50(\mathrm{~d}, J=1.8 \mathrm{~Hz}, 1 \mathrm{H}), 3.87(\mathrm{~s}, 3 \mathrm{H}), 3.53(\mathrm{dd}, J=$ $17.0,2.6 \mathrm{~Hz}, 1 \mathrm{H}), 3.06(\mathrm{dd}, J=17.0,2.8 \mathrm{~Hz}, 1 \mathrm{H}), 2.67-2.59(\mathrm{~m}, 1 \mathrm{H}), 2.12(\mathrm{t}, J=2.7 \mathrm{~Hz}$, $1 \mathrm{H}), 1.47(\mathrm{t}, J=7.13 \mathrm{~Hz}, 3 \mathrm{H}) .{ }^{13} \mathrm{C}$ NMR $\left(100 \mathrm{MHz}, \mathrm{CDCl}_{3}\right) \delta 170.6,159.3(\mathrm{q}, J=37.1$ $\mathrm{Hz}), 142.8,132.8,130.3,128.7,128.2,127.5,126.3,122.3,115.8$ (q, $J=286.3 \mathrm{~Hz}), 79.4$, 72.0, 64.0, $61.4(\mathrm{q}, J=3.2 \mathrm{~Hz}), 53.1,36.5,29.6,21.4$. HRMS $\left(\mathrm{CI} / \mathrm{NH}_{3}\right) \mathrm{m} / \mathrm{z}$ calculated for $\left[\mathrm{C}_{19} \mathrm{H}_{17} \mathrm{NO}_{3} \mathrm{BrF}_{3}+\mathrm{Na}\right] 466.0242$, found 466.0247. IR (thin film) $v_{\max } 3301,1747$, $1694,1204,1154 \mathrm{~cm}^{-1} \cdot[\alpha]_{\mathrm{D}}^{20}+6.0^{\circ}\left(\mathrm{c}=0.3, \mathrm{CHCl}_{3}\right)$.

$(2 S, 5 R, 6 R)-M e t h y l$<smiles>C=CC[C@]1(C(C)=O)C=C[C@@H](C)[C@H](c2c(F)cccc2Br)N1C(=O)O</smiles>

2-allyl-6-(2-bromophenyl)-5-methyl-1-(2,2,2-trifluoroacetyl)1,2,5,6-tetrahydropyridine-2-carboxylate 18: This compound was synthesized by general procedure 1 and isolated in pure form as a colorless oil. ${ }^{1} \mathrm{H}$ NMR $\left(400 \mathrm{MHz}, \mathrm{CDCl}_{3}\right) \delta 8.07(\mathrm{dd}, J=$ $7.9,1.6 \mathrm{~Hz}, 1 \mathrm{H}), 7.53(\mathrm{dd}, J=7.9,1.3 \mathrm{~Hz}, 1 \mathrm{H}), 7.27-7.23(\mathrm{~m}$, $1 \mathrm{H}), 7.11-7.07(\mathrm{~m}, 1 \mathrm{H}), 5.98-5.83(\mathrm{~m}, 3 \mathrm{H}), 5.45(\mathrm{~d}, J=1.8 \mathrm{~Hz}$, $1 \mathrm{H}), 5.14-5.05(\mathrm{~m}, 2 \mathrm{H}), 3.86(\mathrm{~s}, 3 \mathrm{H}), 3.36$ (dddd, $J=14.0,6.3$, $1.3,1.3 \mathrm{~Hz}, 1 \mathrm{H}), 2.84(\mathrm{dd}, J=14.0,8.62 \mathrm{~Hz}, 1 \mathrm{H}), 2.64-2.57(\mathrm{~m}, 1 \mathrm{H}), 1.42(\mathrm{~d}, J=7.2 \mathrm{~Hz}$, $3 \mathrm{H}) .{ }^{13} \mathrm{C}$ NMR $\left(100 \mathrm{MHz}, \mathrm{CDCl}_{3}\right) \delta 171.4,159.0(\mathrm{q}, J=36.4 \mathrm{~Hz}), 143.0,133.2,132.7$, $129.1,128.6,128.2,127.4,127.0,122.3,119.2,115.9(\mathrm{q}, J=286.5 \mathrm{~Hz}), 65.7,61.2(\mathrm{q}, J=$ $3.2 \mathrm{~Hz}), 52.9,43.3,36.3,20.4$. HRMS $\left(\mathrm{CI} / \mathrm{NH}_{3}\right) \mathrm{m} / \mathrm{z}$ calculated for $\left[\mathrm{C}_{19} \mathrm{H}_{19} \mathrm{NO}_{3} \mathrm{~F}_{3} \mathrm{Br}+\mathrm{Na}\right]$ 468.0398 , found 468.0429 . IR (thin film) $v_{\max } 3076,1746,1694,1207,1153 \mathrm{~cm}^{-1} .[\alpha]_{\mathrm{D}}^{20}$ $+27.8^{\circ}\left(\mathrm{c}=1.0, \mathrm{CHCl}_{3}\right)$. 
<smiles>CC(=O)N1[C@@H](c2c(Br)cccc2Br)[C@@H](C)C=C[C@]1(C)C/C=C/P</smiles>
by general procedure 1 and isolated in pure form as a colorless oil. ${ }^{1} \mathrm{H}$ NMR $\left(400 \mathrm{MHz}, \mathrm{CDCl}_{3}\right) \delta 8.06(\mathrm{dd}, J=7.9,1.6 \mathrm{~Hz}, 1 \mathrm{H})$, $7.53(\mathrm{dd}, J=7.9,1.3 \mathrm{~Hz}, 1 \mathrm{H}), 7.37-7.23(\mathrm{~m}, 6 \mathrm{H}), 7.09$ (ddd, $J=$ 7.7, 7.7, $1.6 \mathrm{~Hz}, 1 \mathrm{H}), 6.40(\mathrm{~d}, J=15.9 \mathrm{~Hz}, 1 \mathrm{H}), 6.30-6.22(\mathrm{~m}$, $1 \mathrm{H}), 5.98(\mathrm{dd}, J=10.3,6.6 \mathrm{~Hz}, 1 \mathrm{H}), 5.90(\mathrm{~d}, J=10.3 \mathrm{~Hz}, 1 \mathrm{H})$, $5.48(\mathrm{~d}, J=1.2 \mathrm{~Hz}, 1 \mathrm{H}), 3.89(\mathrm{~s}, 3 \mathrm{H}), 3.49$ (ddd, $J=13.9,6.5$, $1.2 \mathrm{~Hz}, 1 \mathrm{H}), 3.07(\mathrm{dd}, J=14.0,8.5 \mathrm{~Hz}, 1 \mathrm{H}), 2.60$ (app. dp, $J=$ 7.1, $1.4 \mathrm{~Hz}, 1 \mathrm{H}), 1.40(\mathrm{~d}, J=7.3 \mathrm{~Hz}, 3 \mathrm{H}) .{ }^{13} \mathrm{C}$ NMR $\left(100 \mathrm{MHz}, \mathrm{CDCl}_{3}\right) \delta 171.3,159.0$ $(\mathrm{q}, J=36.3 \mathrm{~Hz}), 143.0,137.0,134.2,132.7,129.1,128.6,128.5,128.2,127.4,127.3$, $127.0,126.2,124.7,122.3,116.0(\mathrm{q}, J=286.5 \mathrm{~Hz}), 66.1,61.1(\mathrm{q}, J=3.3 \mathrm{~Hz}), 53.0,42.5$, 36.2, 20.4. HRMS $\left(\mathrm{CI} / \mathrm{NH}_{3}\right) \mathrm{m} / \mathrm{z}$ calculated for $\left[\mathrm{C}_{25} \mathrm{H}_{23} \mathrm{NO}_{3} \mathrm{~F}_{3} \mathrm{Br}+\mathrm{Na}\right] 544.0711$, found 544.0735. IR (thin film) $v_{\max } 3028,1746,1693,1207,1152 \mathrm{~cm}^{-1}$. $[\alpha]_{\mathrm{D}}^{20}+57.2^{\circ}(\mathrm{c}=1.0$, $\left.\mathrm{CHCl}_{3}\right)$.

(2S,5R,6R)-Methyl 6-(2-bromophenyl)-5-methyl-2-(2-phenylallyl)-1-(2,2,2-trifluoro-<smiles>C=C(C[C@]1(C)C=C[C@@H](C)[C@@H](c2c(Br)cccc2Br)N1C(C)=O)c1ccccc1</smilesacetyl)-1,2,5,6-tetrahydropyridine-2-carboxylate 20: This compound was synthesized by general procedure 1 and isolated in pure form as a colorless oil. ${ }^{1} \mathrm{H}$ NMR $\left(400 \mathrm{MHz}, \mathrm{CDCl}_{3}\right) \delta$ 7.91-7.89 (m, $1 \mathrm{H})$, 7.52-7.50 (m, 2H), 7.40-7.24 (m, 5H), 7.11$7.09(\mathrm{~m}, 1 \mathrm{H}), 5.69(\mathrm{dd}, J=10.3,1.4 \mathrm{~Hz}, 1 \mathrm{H}), 5.62(\mathrm{dd}, J=10.2$, $4.6 \mathrm{~Hz}, 1 \mathrm{H}), 5.33(\mathrm{~d}, J=1.5 \mathrm{~Hz}, 1 \mathrm{H}), 5.18(\mathrm{~b} \mathrm{~s}, 1 \mathrm{H}), 5.08(\mathrm{~d}, J=$ $4.7 \mathrm{~Hz}, 1 \mathrm{H}), 3.74(\mathrm{~d}, J=14.3 \mathrm{~Hz}, 1 \mathrm{H}), 3.65(\mathrm{~s}, 3 \mathrm{H}), 3.40(\mathrm{~d}, J=14.3 \mathrm{~Hz}, 1 \mathrm{H}), 2.52-2.43$ $(\mathrm{m}, 1 \mathrm{H}), 1.12(\mathrm{~d}, J=7.26 \mathrm{~Hz}, 3 \mathrm{H}) \cdot{ }^{13} \mathrm{C}$ NMR $\left(100 \mathrm{MHz}, \mathrm{CDCl}_{3}\right) \delta 171.1,160.1(\mathrm{q}, J=$ $37 \mathrm{~Hz}), 144.1,142.6,142.3,132.7,129.8,128.8,128.8,128.0,127.9,127.3,127.1,123.6$, $115.7(\mathrm{q}, J=286.5 \mathrm{~Hz}), 119.9,67.0,61.6(\mathrm{q}, J=2.3 \mathrm{~Hz}), 52.6,42.0,37.7$, 19.4. HRMS $\left(\mathrm{CI} / \mathrm{NH}_{3}\right) \mathrm{m} / \mathrm{z}$ calculated for $\left[\mathrm{C}_{25} \mathrm{H}_{23} \mathrm{NO}_{3} \mathrm{~F}_{3} \mathrm{Br}+\mathrm{Na}\right]$ 544.0711, found 544.0724. IR (thin film) $v_{\max } 2953,1742,1689,1202,1157 \mathrm{~cm}^{-1} .[\alpha]_{\mathrm{D}}{ }^{20}+27.9^{\circ}\left(\mathrm{c}=1.0, \mathrm{CHCl}_{3}\right)$.

\section{(2S,5R,6R)-Methyl 6-(2-bromophenyl)-5-methyl-2-((E)-pent-2-enyl)-1-(2,2,2-triflu-

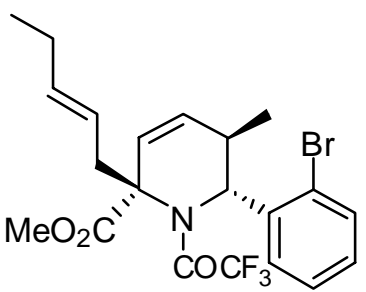 oroacetyl)-1,2,5,6-tetrahydropyridine-2-carboxylate 21 : This compound was synthesized by general procedure 1 and isolated in pure form as a colorless oil. ${ }^{1} \mathrm{H}$ NMR $(400 \mathrm{MHz}$, $\left.\mathrm{CDCl}_{3}\right) \delta 8.01(\mathrm{dd}, J=7.9,1.3 \mathrm{~Hz}, 1 \mathrm{H}), 7.52(\mathrm{dd}, J=7.9,1.2$ $\mathrm{Hz}, 1 \mathrm{H}), 7.26-7.22(\mathrm{~m}, 1 \mathrm{H}), 7.11-7.07(\mathrm{~m}, 1 \mathrm{H}), 5.93(\mathrm{dd}, J=$ $10.2,6.4 \mathrm{~Hz}, 1 \mathrm{H}), 5.83(\mathrm{~d}, J=10.3 \mathrm{~Hz}, 1 \mathrm{H}), 5.54-5.38(\mathrm{~m}, 3 \mathrm{H})$, $3.84(\mathrm{~s}, 3 \mathrm{H}), 3.10(\mathrm{dd}, J=14.2,5.9 \mathrm{~Hz}, 1 \mathrm{H}), 2.97(\mathrm{dd}, J=14.2$,} $8.1 \mathrm{~Hz}, 1 \mathrm{H}), 2.60-2.53(\mathrm{~m}, 1 \mathrm{H}), 2.07-2.0(\mathrm{~m}, 2 \mathrm{H}), 1.39(\mathrm{~d}, J=7.3 \mathrm{~Hz}, 3 \mathrm{H}), 0.97(\mathrm{t}, J=$ $7.45 \mathrm{~Hz}, 3 \mathrm{H}){ }^{13} \mathrm{C}$ NMR $\left(100 \mathrm{MHz}, \mathrm{CDCl}_{3}\right) \delta 171.4,158.9(\mathrm{~d}, J=36.5 \mathrm{~Hz}), 143.1,137.3$, 132.7, 128.9, 128.6, 128.2, 127.3, 127.1, 123.1, 122.5, 116.0 (d, $J=288.4 \mathrm{~Hz}$,), 66.3, $61.1(\mathrm{q}, J=3.2 \mathrm{~Hz}), 52.8,41.3,36.4,25.7,20.3,13.5$. HRMS $\left(\mathrm{CI} / \mathrm{NH}_{3}\right) \mathrm{m} / \mathrm{z}$ calculated for $\left[\mathrm{C}_{21} \mathrm{H}_{23} \mathrm{NO}_{3} \mathrm{BrF}_{3}+\mathrm{Na}\right]$ 496.0711, found 496.0726. IR (thin film) $v_{\max } 2964,1746$, $1696,1202,1153 \mathrm{~cm}^{-1} \cdot[\alpha]_{\mathrm{D}}^{20}+33.1^{\circ}\left(\mathrm{c}=1.0, \mathrm{CHCl}_{3}\right)$. 
(2R,5S,6S)-Methyl 2-(2-ethynylbenzyl)-5-methyl-6-phenyl-1-(2,2,2-trifluoroacetyl)-

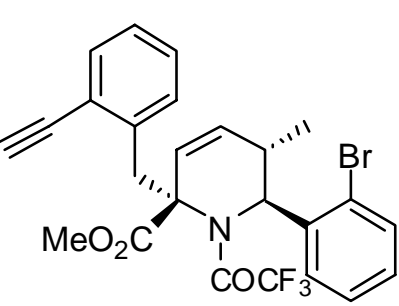

1,2,5,6-tetrahydropyridine-2-carboxylate 22: This compound was synthesized by general procedure 1 and isolated in pure form as a colorless oil. ${ }^{1} \mathrm{H}$ NMR $\left(400 \mathrm{MHz}, \mathrm{CDCl}_{3}\right) \delta$ $7.99(\mathrm{dd}, J=7.9,1.4 \mathrm{~Hz}, 1 \mathrm{H}), 7.50(\mathrm{~d}, J=8.0 \mathrm{~Hz}, 2 \mathrm{H}), 7.37$ $7.05(\mathrm{~m}, 2 \mathrm{H}), 7.11-7.05(\mathrm{~m}, 2 \mathrm{H}), 6.08(\mathrm{~d}, J=10.5 \mathrm{~Hz}, 1 \mathrm{H})$, $5.82(\mathrm{dd}, J=10.4,6.6 \mathrm{~Hz}, 1 \mathrm{H}), 5.33(\mathrm{~s}, 1 \mathrm{H}), 4.08(\mathrm{~d}, J=14.0$ $\mathrm{Hz}, 1 \mathrm{H}), 4.02(\mathrm{~d}, J=14.0 \mathrm{~Hz}, 1 \mathrm{H}), 3.89(\mathrm{~s}, 3 \mathrm{H}), 3.29(\mathrm{~s}, 1 \mathrm{H})$, 2.26 ( app. p, $J=7.2 \mathrm{~Hz}, 1 \mathrm{H}), 0.31(\mathrm{~d}, J=7.4 \mathrm{~Hz}, 3 \mathrm{H}) .{ }^{13} \mathrm{C} \mathrm{NMR}\left(100 \mathrm{MHz}, \mathrm{CDCl}_{3}\right) \delta$ $170.7,159.5(\mathrm{q}, J=36.1 \mathrm{~Hz}), 143.9,138.4,133.4,132.7,131.7,129.0,128.7,128.4$, $127.8,127.3,127.2,125.9,124.9,122.1,116.0(\mathrm{q}, J=286.9 \mathrm{~Hz}), 83.2,81.2,68.5,60.9$ $(\mathrm{q}, J=3.3 \mathrm{~Hz}), 53.1,38.4,36.4,19.2$. HRMS $\left(\mathrm{CI} / \mathrm{NH}_{3}\right) \mathrm{m} / \mathrm{z}$ calculated for [C25H21NO3NaBrF3] 542.0555, found 542.0566. IR (thin film) $v_{\max } 2955,1748,1694$, $1198,1153 \mathrm{~cm}^{-1} .[\alpha]_{\mathrm{D}}^{20}-76.0^{\circ}\left(\mathrm{c}=1.0, \mathrm{CHCl}_{3}\right)$.

(2R,5S,6S)-Methyl 2-(2-bromobenzyl)-5-methyl-6-phenyl-1-(2,2,2-trifluoroacetyl)1,2,5,6-tetrahydropyridine-2-carboxylate 23: This compound<smiles>CC(=O)[C@H](Cc1ccccc1Br)N1C=C[C@@H](C)C(F)(c2ccccc2)OC1=O</smiles>
was synthesized by general procedure 1 and isolated in pure form as a colorless oil. ${ }^{1} \mathrm{H}$ NMR $\left(400 \mathrm{MHz}, \mathrm{CDCl}_{3}\right) \delta 7.56(\mathrm{~d}, J=8.0$ $\mathrm{Hz}, 1 \mathrm{H}), 7.40(\mathrm{~d}, J=6.8 \mathrm{~Hz}, 2 \mathrm{H}), 7.29-7.24(\mathrm{~m}, 4 \mathrm{H}), 7.13-7.07$ (m, $2 \mathrm{H}), 6.19(\mathrm{~d}, J=10.4 \mathrm{~Hz}, 1 \mathrm{H}), 6.08(\mathrm{dd}, J=10.2,6.4 \mathrm{~Hz}, 1 \mathrm{H})$, $4.94(\mathrm{~s}, 1 \mathrm{H}), 4.15(\mathrm{~d}, J=14.4 \mathrm{~Hz}, 1 \mathrm{H}), 3.85(\mathrm{~d}, J=14.3 \mathrm{~Hz}, 1 \mathrm{H})$, 3.39 (s, 3H), 2.57 (app. p, $J=6.6 \mathrm{~Hz}, 1 \mathrm{H}), 0.38(\mathrm{~d}, J=7.12 \mathrm{~Hz}$, $3 \mathrm{H}) .{ }^{13} \mathrm{C}$ NMR $\left(100 \mathrm{MHz}, \mathrm{CDCl}_{3}\right) \delta 169.9,159.3(\mathrm{q}, J=34.9 \mathrm{~Hz}), 140.1,135.5,133.3$, $132.7,130.4,128.9,128.4,127.8,127.6,127.2,127.1,126.7,116.6(\mathrm{q}, J=287.8 \mathrm{~Hz})$, $67.8,61.3(\mathrm{q}, J=2.8 \mathrm{~Hz}), 52.4,38.7,33.7,18.2$. HRMS $\left(\mathrm{CI} / \mathrm{NH}_{3}\right) \mathrm{m} / \mathrm{z}$ calculated for $\left[\mathrm{C}_{23} \mathrm{H}_{21} \mathrm{NO}_{3} \mathrm{BrF}_{3}+\mathrm{Na}\right]$ 518.0555, found 518.0519. IR (thin film) $v_{\max } 2953,1732,1693$, $1263,1190 \mathrm{~cm}^{-1} \cdot[\alpha]_{\mathrm{D}}^{20}-41.5^{\circ}\left(\mathrm{c}=1.0, \mathrm{CHCl}_{3}\right)$.

(2R,5S,6S)-Methyl 2-allyl-5-methyl-6-phenyl-1-(2,2,2-trifluoroacetyl)-1,2,5,6-tetra-<smiles>C=C[C@]1(CC(C)=O)C=C[C@@H](C)C(c2ccccc2[O+])N1C(=O)OC</smileshydropyridine-2-carboxylate 24: This compound was synthesized by general procedure 1 and isolated in pure form as a colorless oil. ${ }^{1} \mathrm{H}$ NMR $\left(400 \mathrm{MHz}, \mathrm{CDCl}_{3}\right) \delta$ 7.42-7.39 (m, $\left.2 \mathrm{H}\right)$, 7.29-7.21 (m, 3H), $6.24(\mathrm{dd}, J=10.3,6.0 \mathrm{~Hz}, 1 \mathrm{H}), 5.79(\mathrm{dd}, J=$ $10.3,0.9 \mathrm{~Hz}, 1 \mathrm{H}), 5.61$ (dddd, $J=16.9,10.1,9.4,5.6 \mathrm{~Hz}, 1 \mathrm{H}$ ), 5.18-5.07 (m, 3H), $5.01(\mathrm{~s}, 1 \mathrm{H}), 3.56(\mathrm{dd}, J=14.2,9.3 \mathrm{~Hz}, 1 \mathrm{H}), 3.19(\mathrm{~s}, 3 \mathrm{H}), 2.59(\mathrm{dd}, J$ $=14.2,5.5 \mathrm{~Hz}, 1 \mathrm{H}), 1.24(\mathrm{~d}, J=7.1 \mathrm{~Hz}, 3 \mathrm{H}) .{ }^{13} \mathrm{C} \mathrm{NMR}\left(100 \mathrm{MHz}, \mathrm{CDCl}_{3}\right) \delta 170.2$, $158.0(\mathrm{q}, J=34.8 \mathrm{~Hz}), 139.1,131.6,130.7,128.6(\mathrm{q}, J=2.5 \mathrm{~Hz}), 128.0,127.7,127.6$, $116.6(\mathrm{q}, J=287.6 \mathrm{~Hz}), 119.8,65.3,60.5(\mathrm{q}, J=2.6 \mathrm{~Hz}), 52.1,39.8,33.1$, 19.2. HRMS $\left(\mathrm{CI} / \mathrm{NH}_{3}\right) \mathrm{m} / \mathrm{z}$ calculated for $\left[\mathrm{C}_{19} \mathrm{H}_{20} \mathrm{NO}_{3} \mathrm{~F}_{3}+\mathrm{Na}\right] 390.1293$, found 390.1330. IR (thin film) $v_{\max } 2953,1729,1702,1262,1146 \mathrm{~cm}^{-1} .[\alpha]_{\mathrm{D}}{ }^{20}-52.1^{\circ}\left(\mathrm{c}=1.0, \mathrm{CHCl}_{3}\right)$. 
3. ${ }^{1} \mathrm{H}$ and ${ }^{13} \mathrm{C}$ NMR Spectra of Alkylated Tetrahydropyridines.

${ }^{1} \mathrm{H}$ NMR of 15.
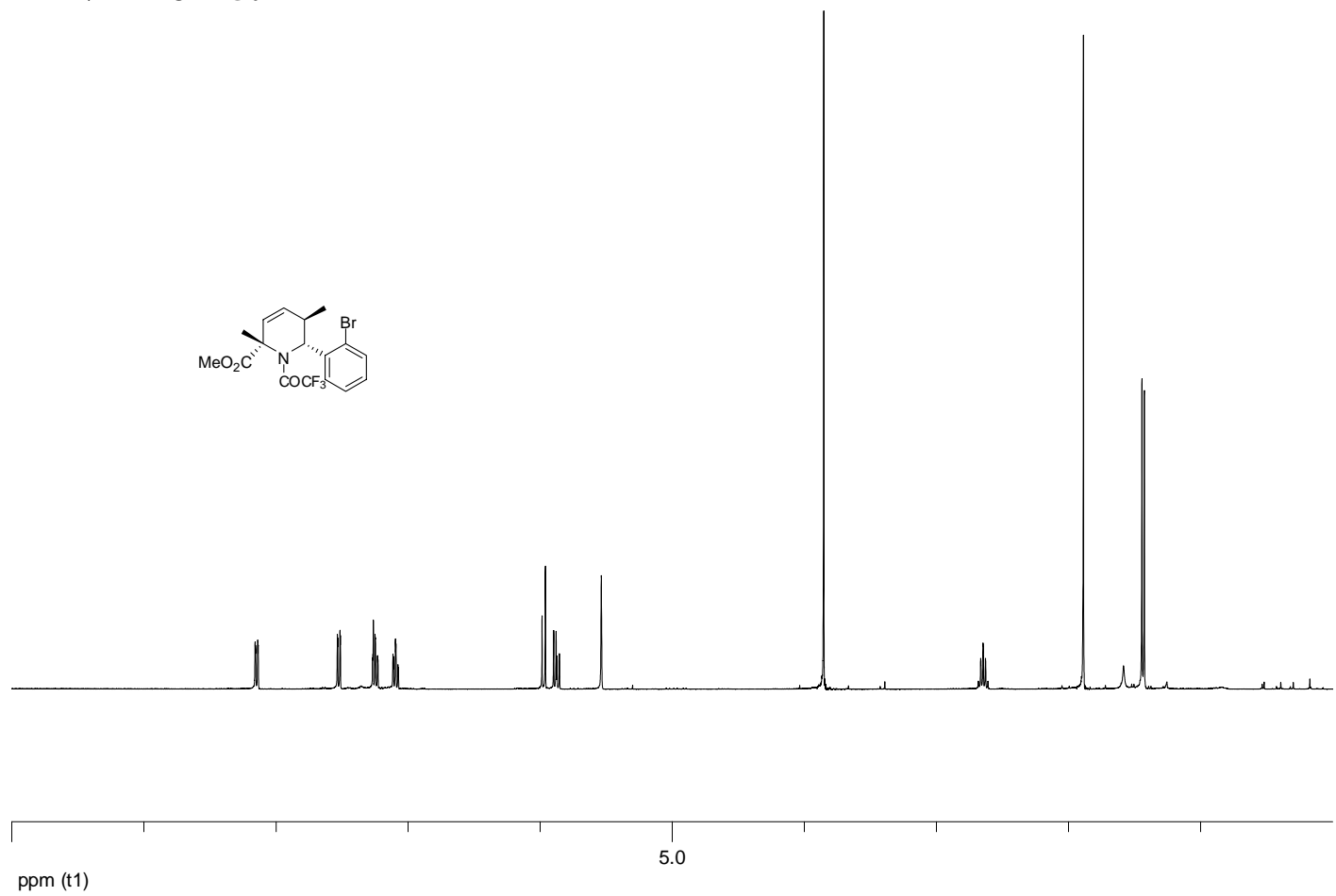

ppm (t1)

${ }^{13}$ C NMR of 15.

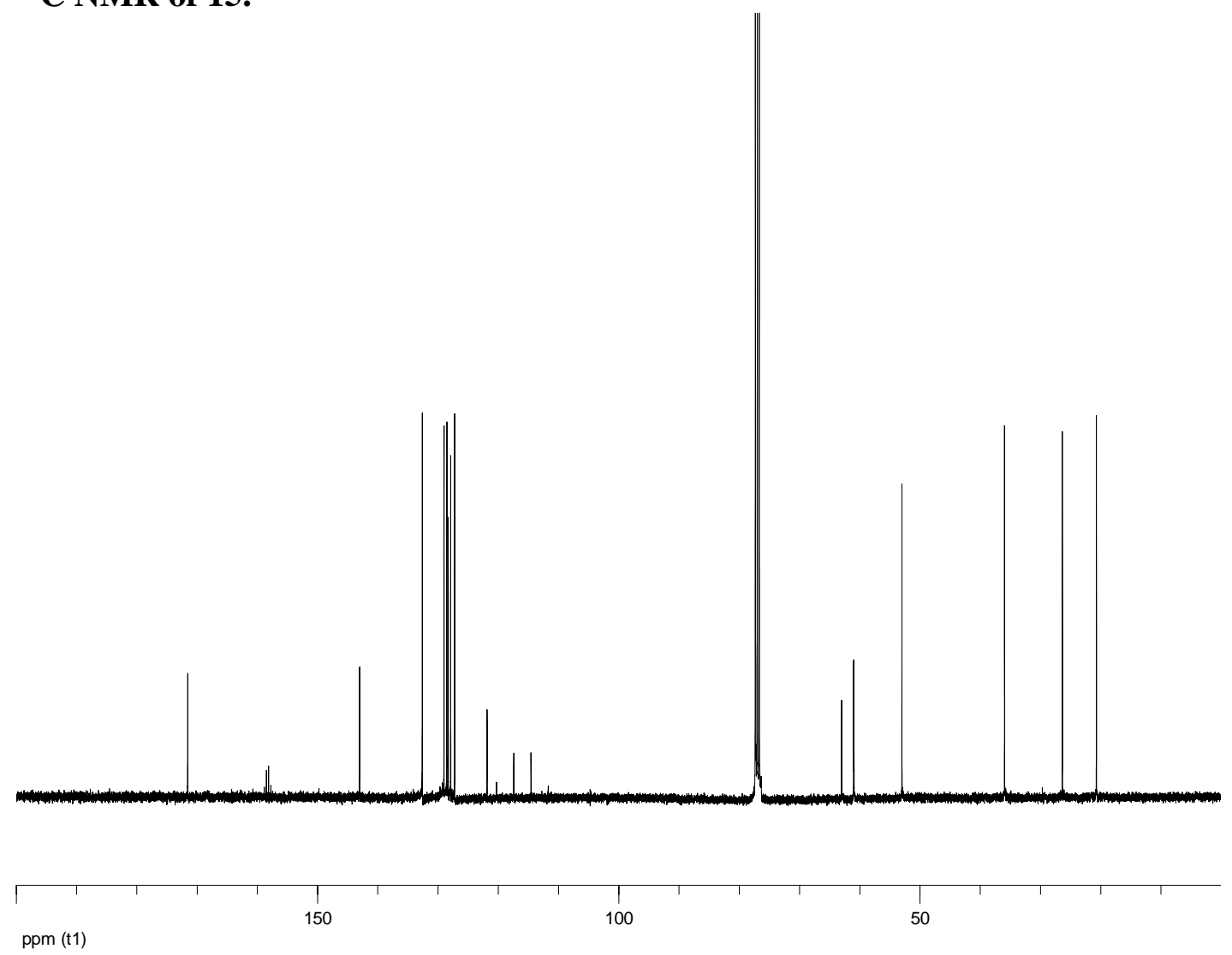


1D NOE Spectrum of 15 indicating a syn relationship of the two methyl groups: (doublet at 1.43 ppm (Peak 1) irradiated)
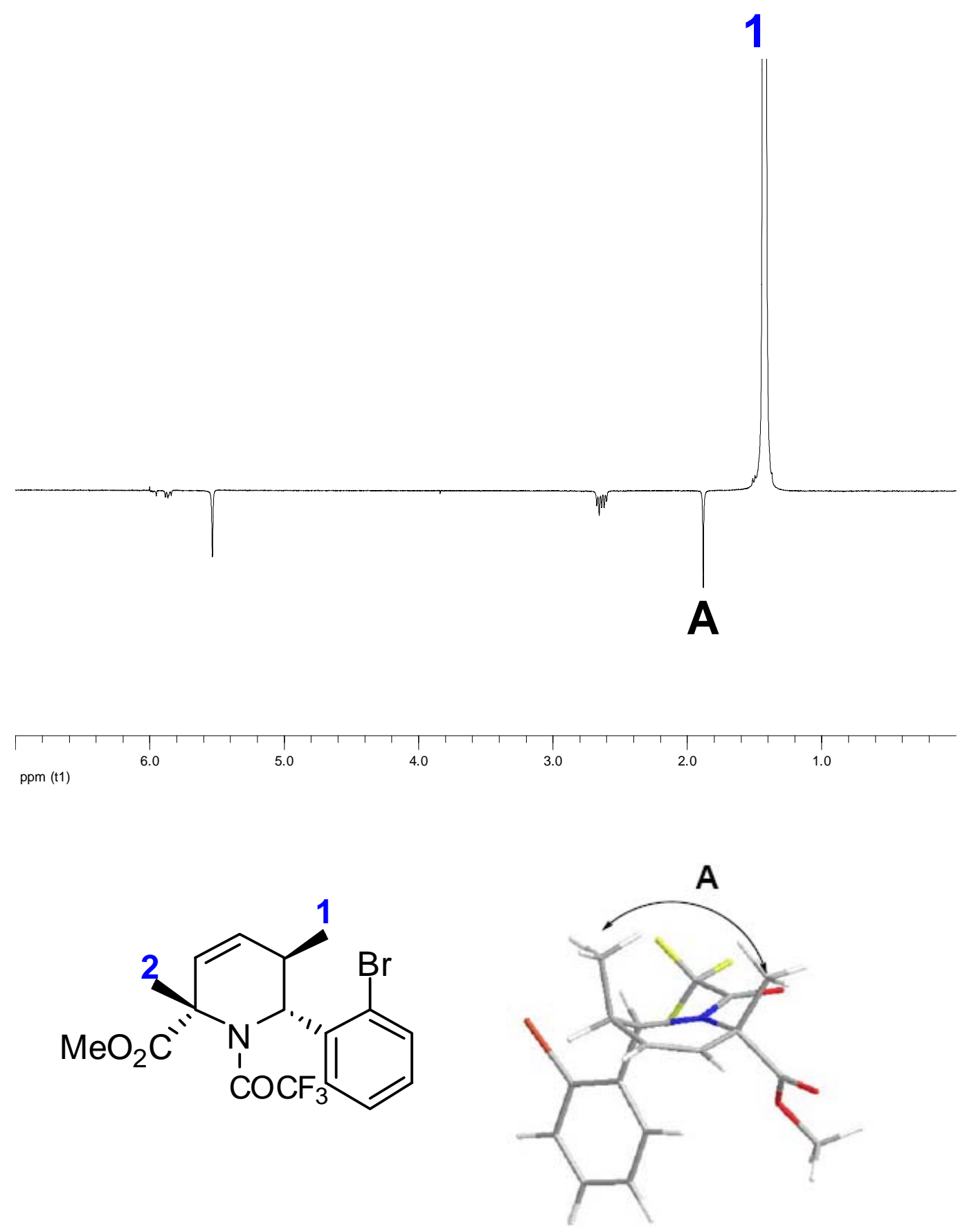
1D NOE Spectrum of 15 indicating a syn relationship of the two methyl groups: (Singlet at 1.89 ppm (Peak 2) irradiated)
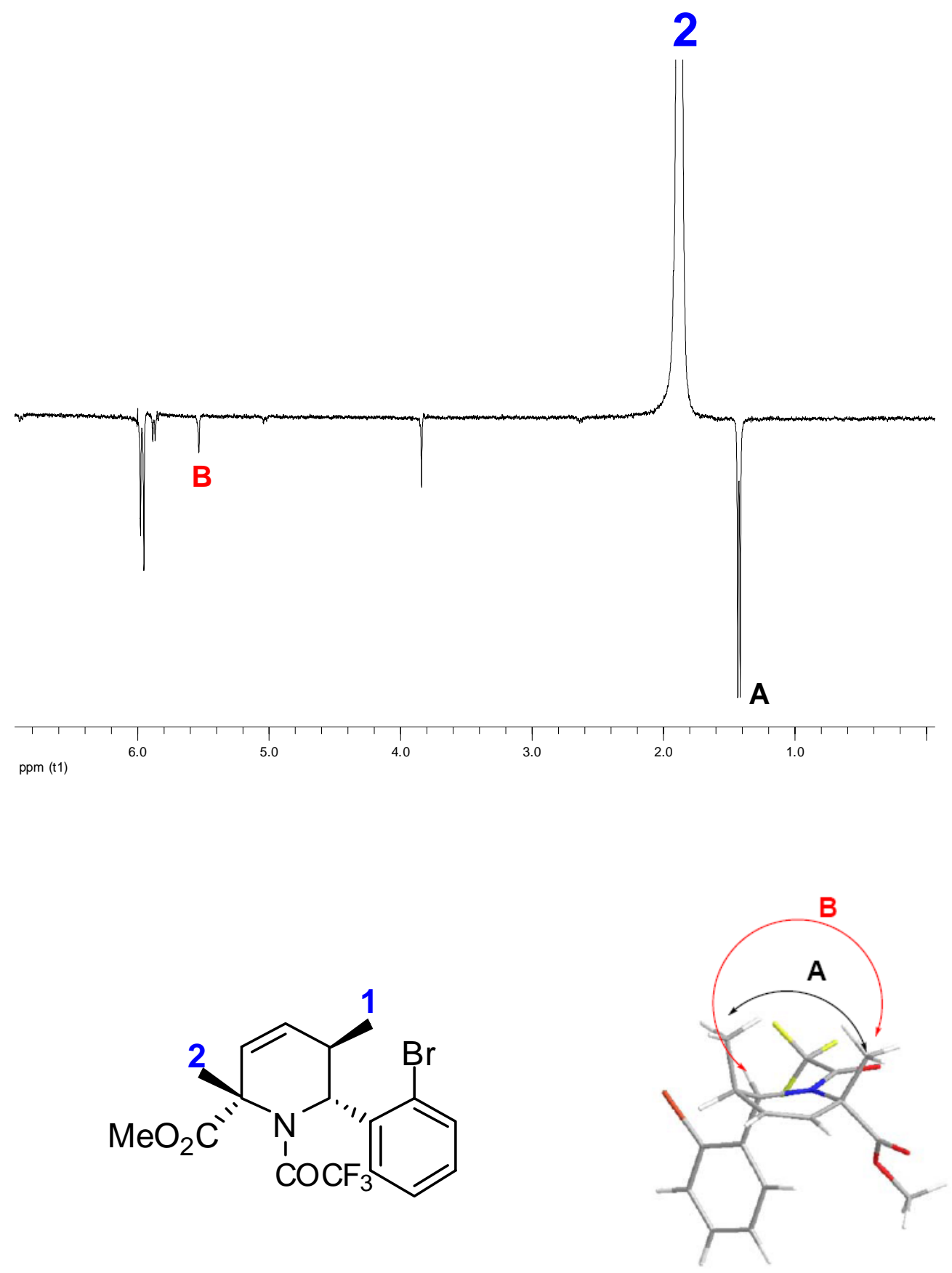
${ }^{1}$ H NMR Spectrum of 16.

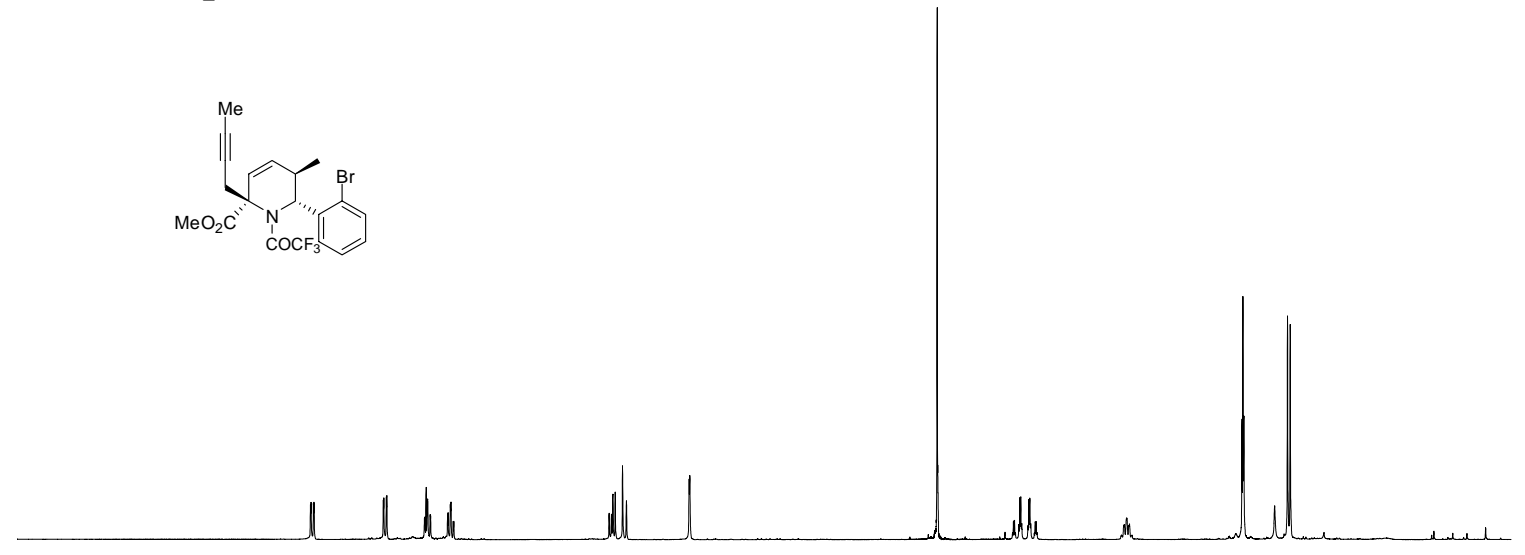

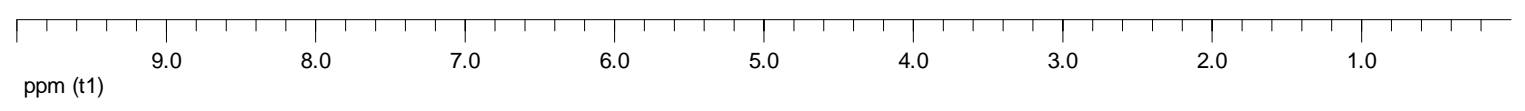

\section{${ }^{13}$ C NMR Spectrum of 16.}

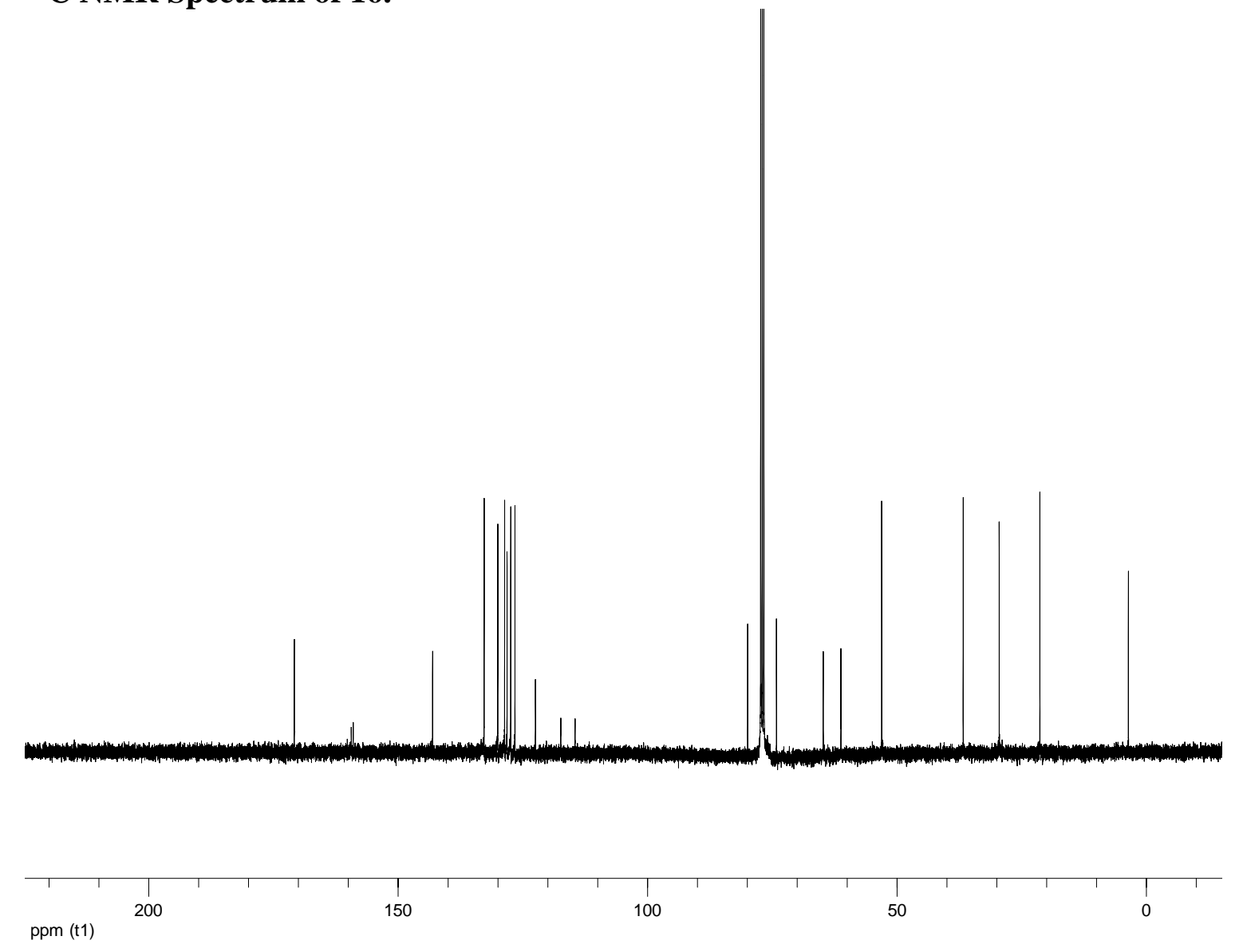


Expanded Segment of NOESY Spectrum of 16.
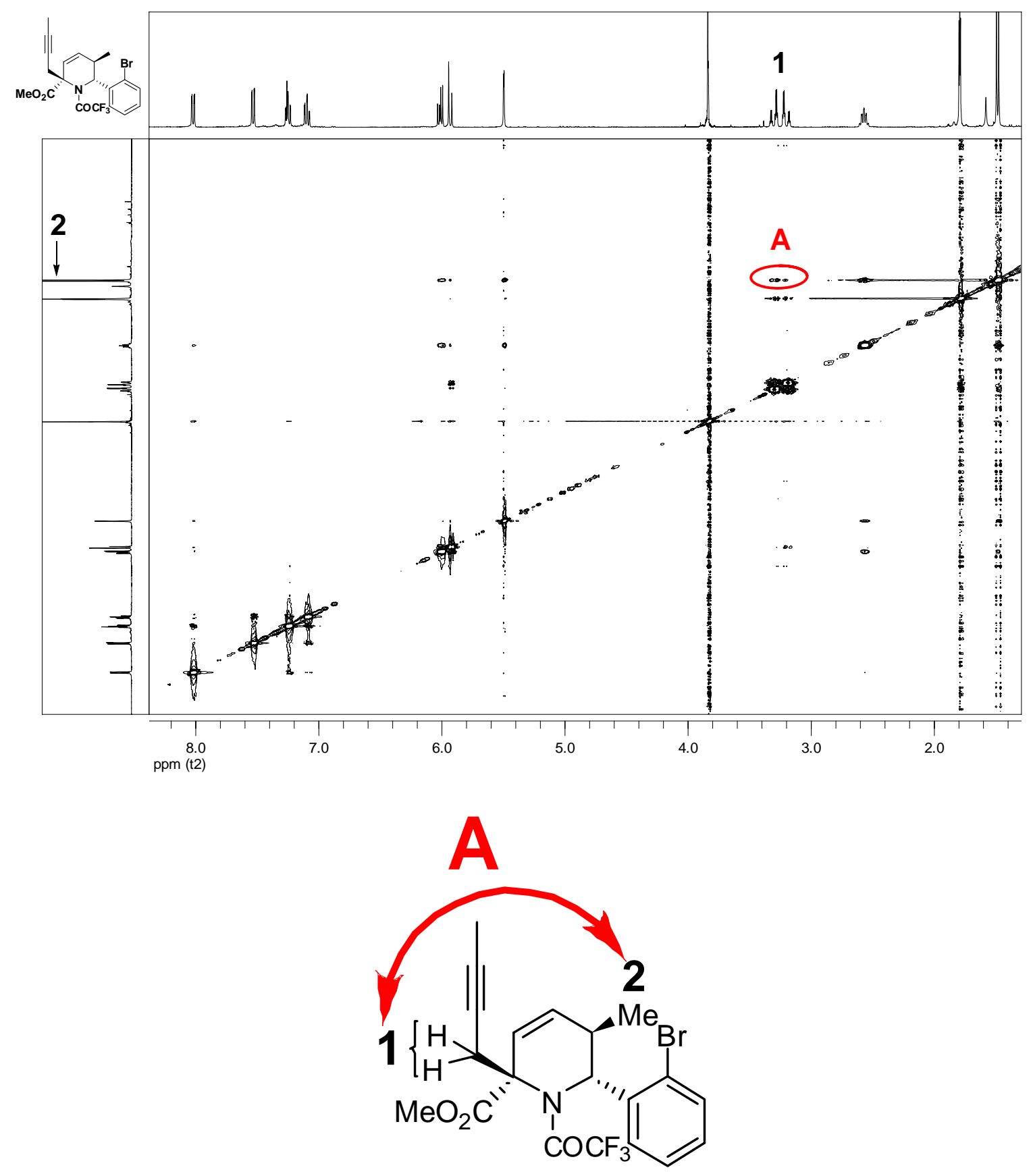
${ }^{1} \mathbf{H}$ NMR Spectrum of 17.<smiles>CC#CC[C@]1(O)C=C[C@@H](C)[C@H](c2ccccc2Br)N1C(C)(F)F</smiles>
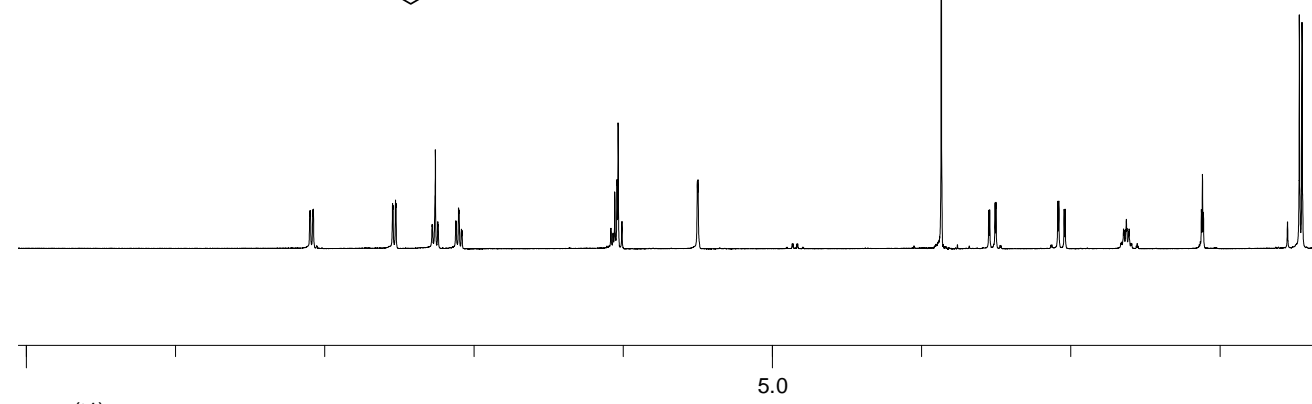

ppm (t1)

${ }^{13}$ C NMR Spectrum of 17.

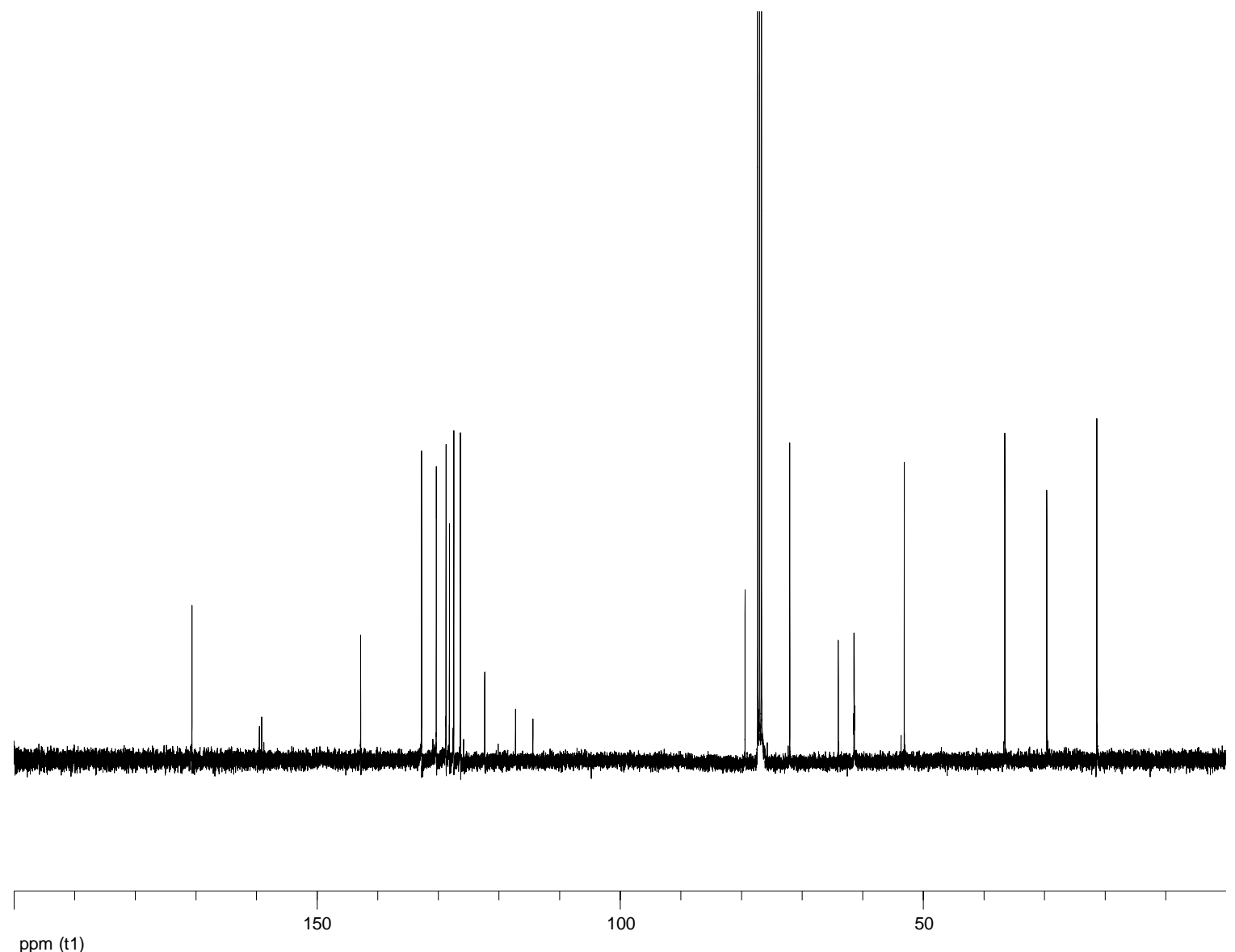

ppm (t1)

SI-11 
${ }^{1} \mathrm{H}$ NMR Spectrum of 18.
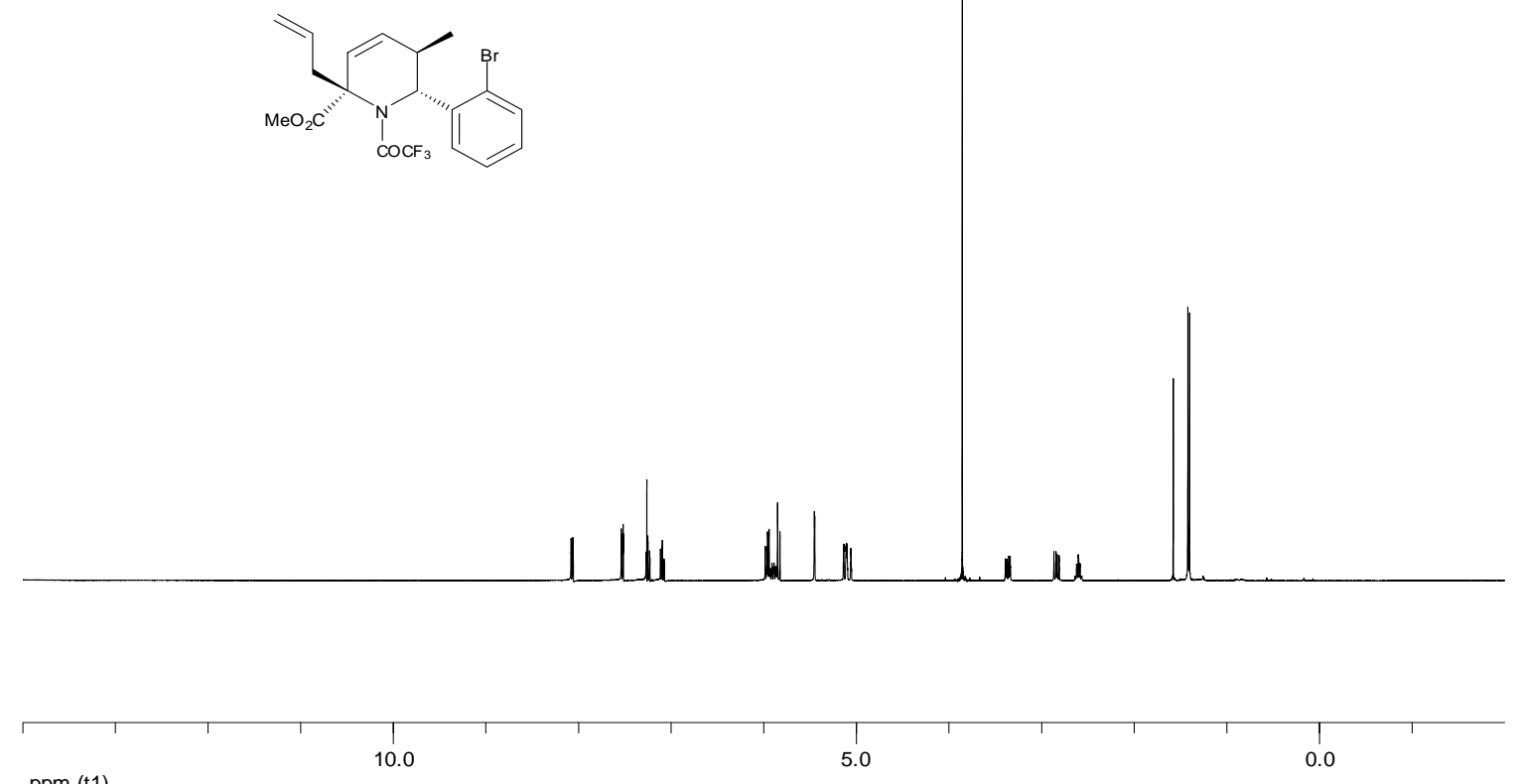

${ }^{\text {ppm }}$ (11) NMR Spectrum of 18.
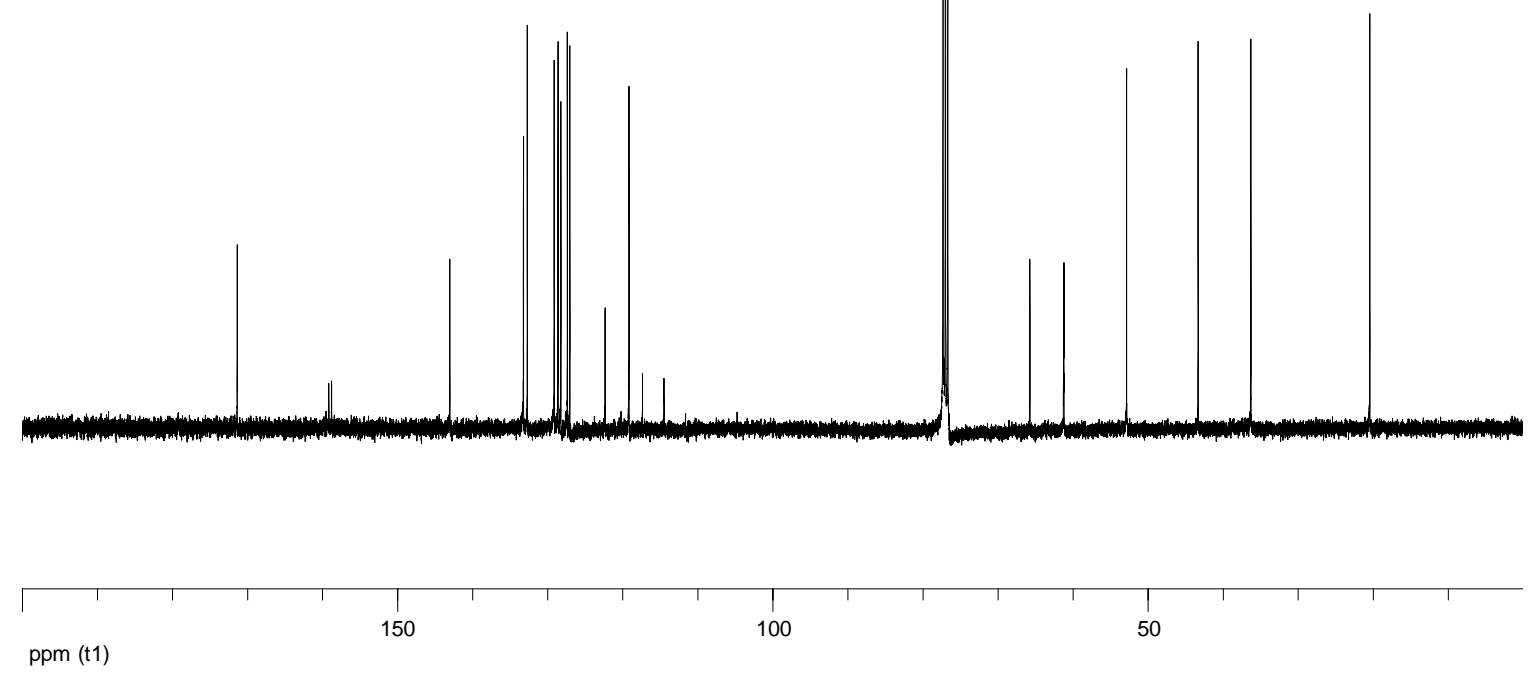

SI-12 
${ }^{1}$ H NMR Spectrum of 19.
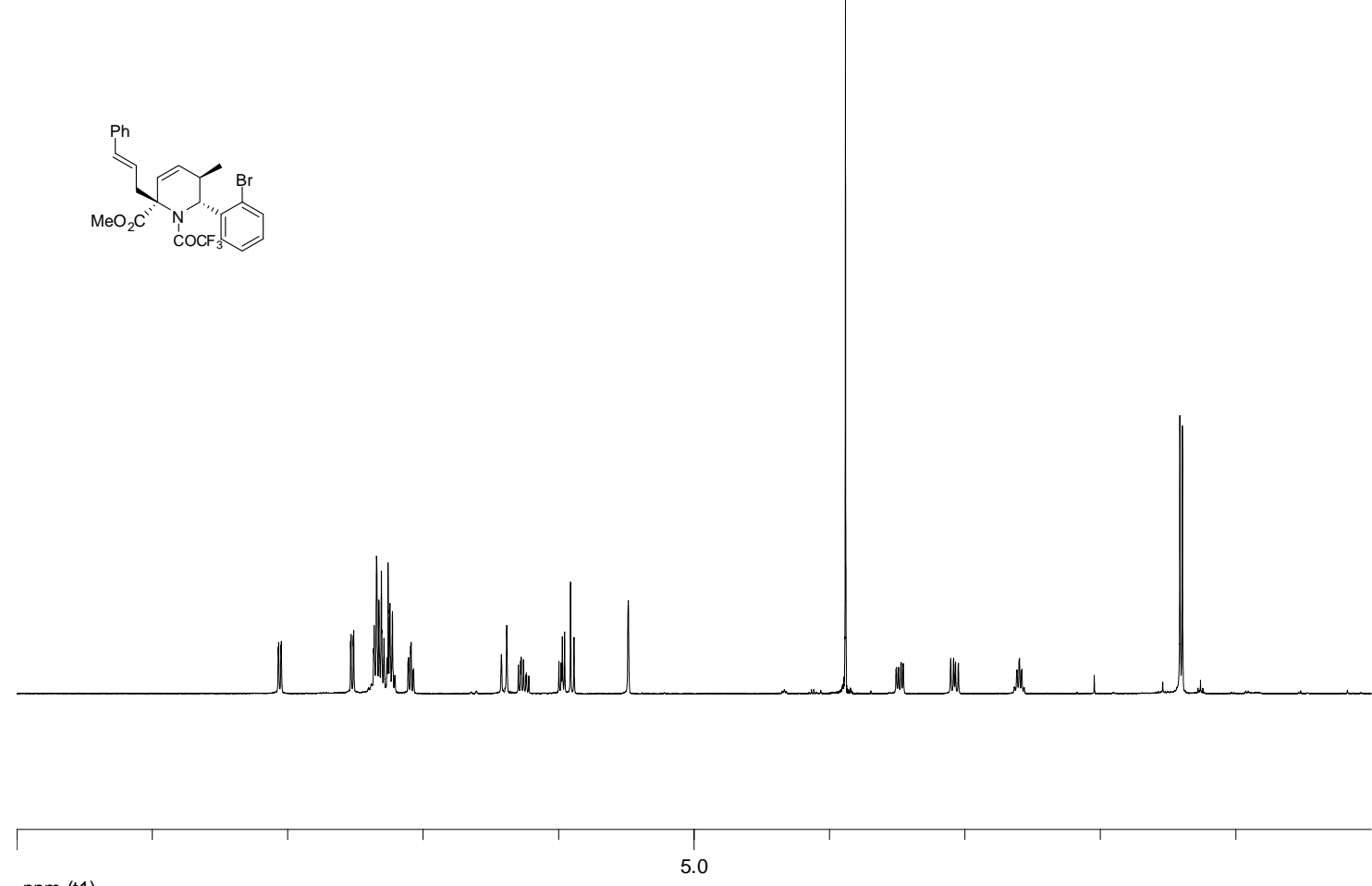

ppm (t1)

${ }^{13}$ C NMR Spectrum of 19.

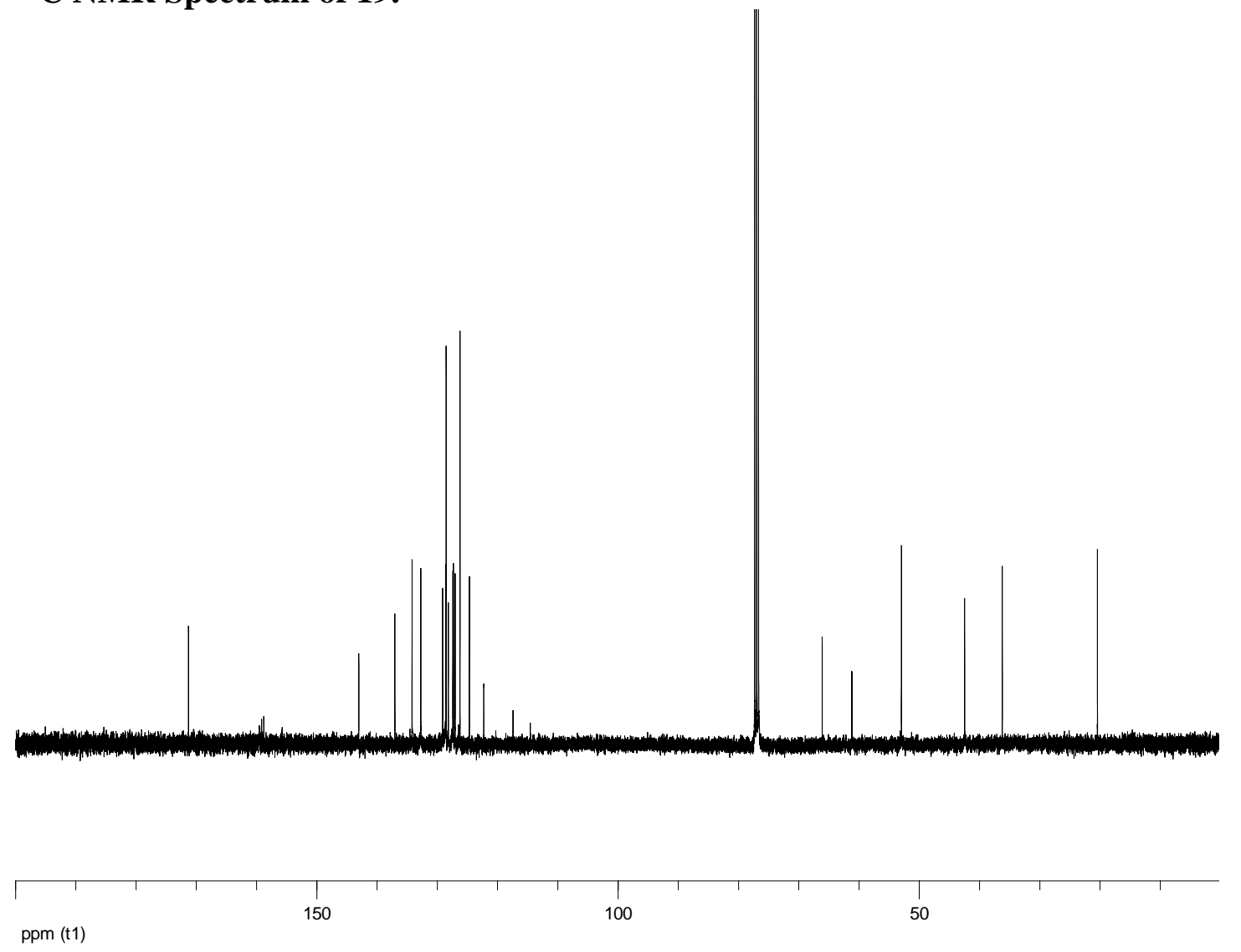

SI-13 
${ }^{1}$ H NMR Spectrum of 20.
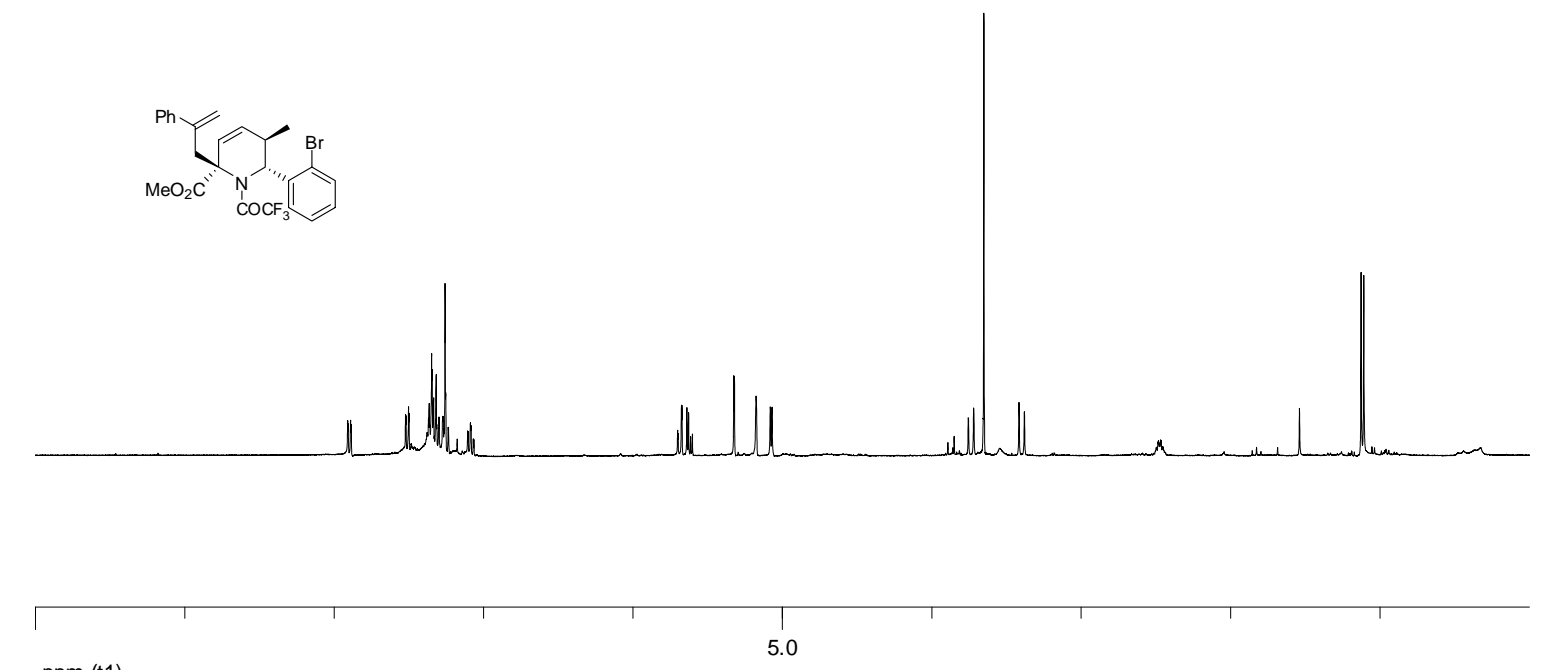

ppm (t1)

${ }^{13}$ C NMR Spectrum of 20.

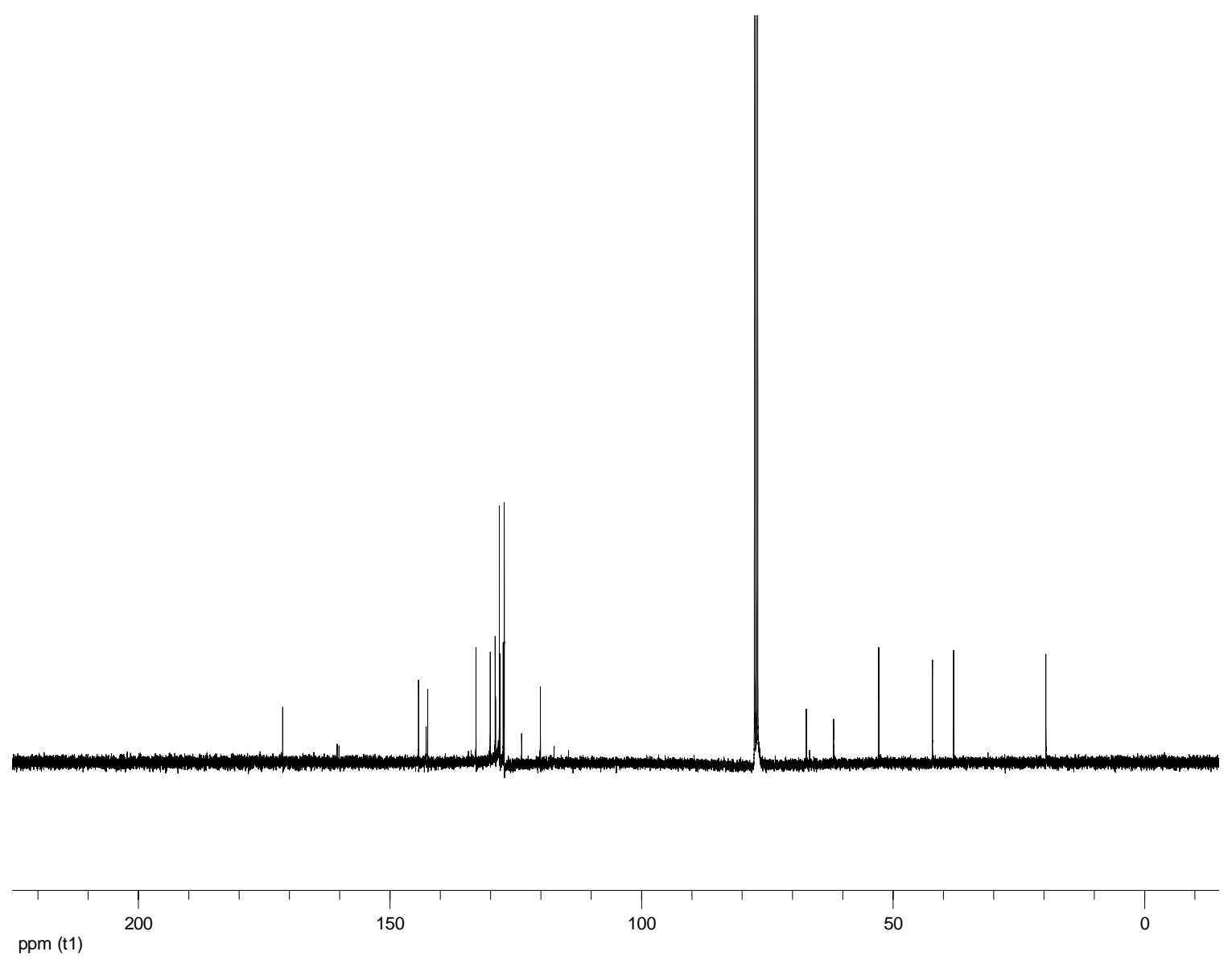

SI-14 
${ }^{1} \mathrm{H}$ NMR Spectrum of 21.
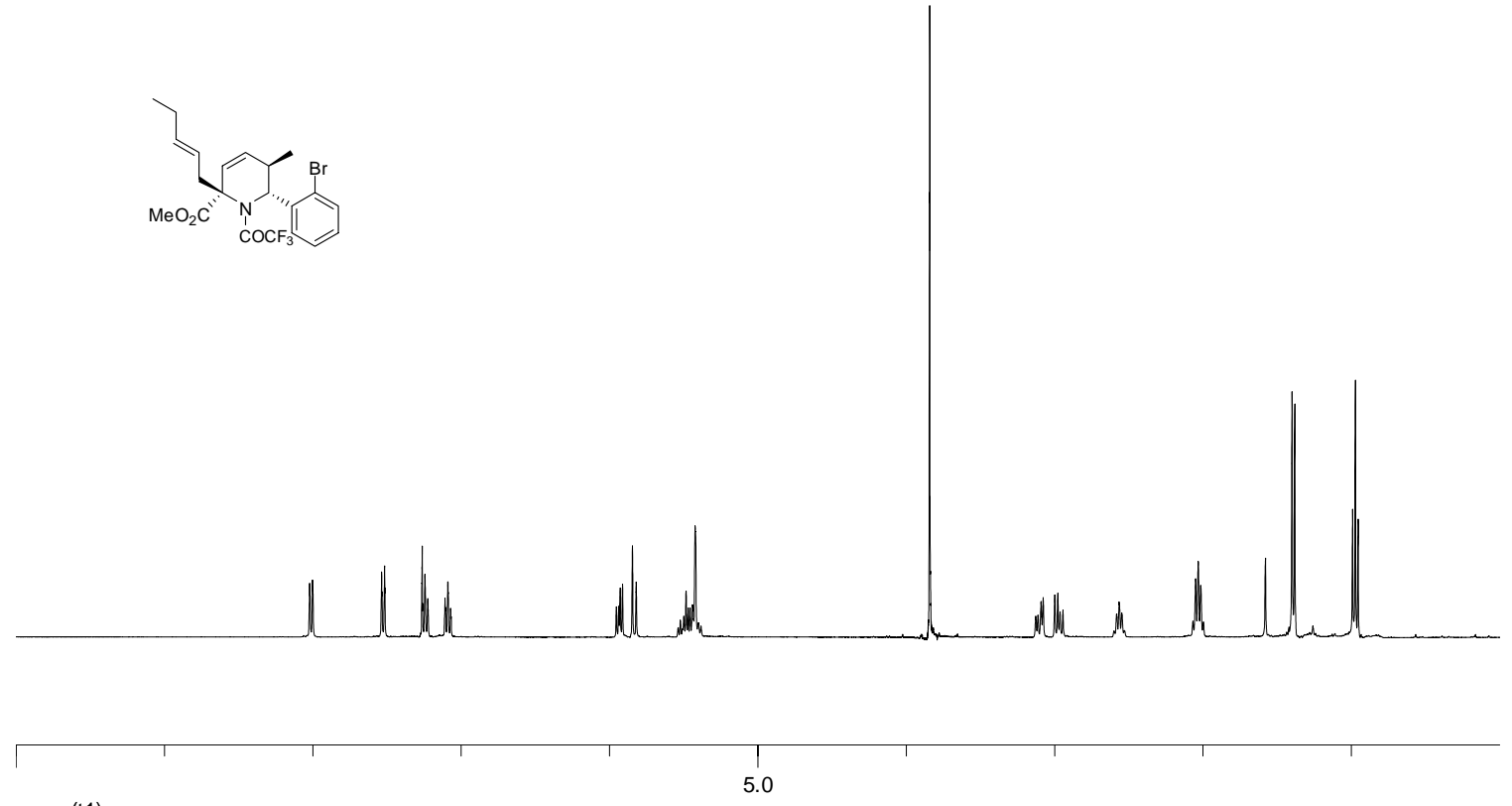

ppm (t1)

${ }^{13}$ C NMR of 21.
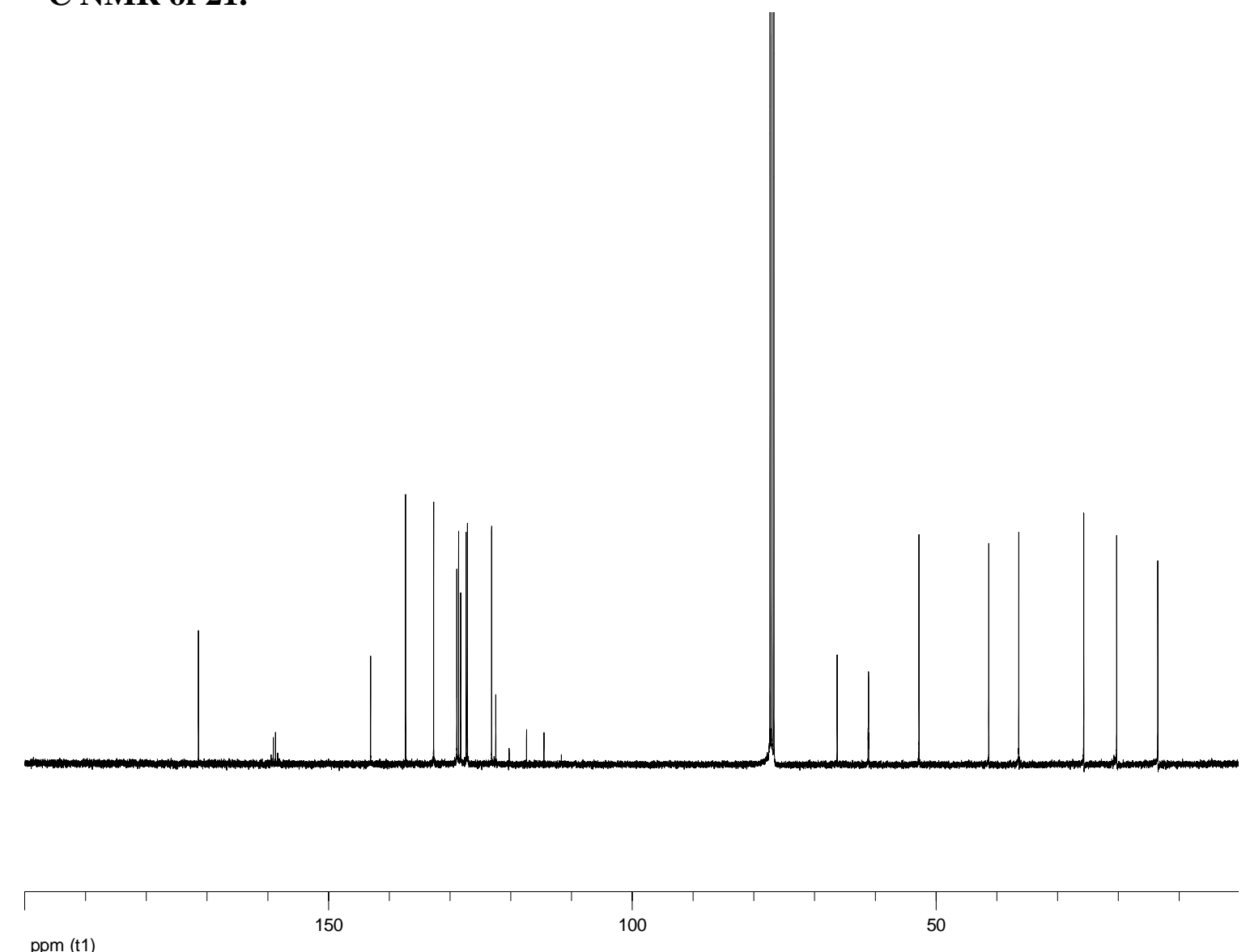

ppm (t1)

SI-15 
${ }^{1} \mathrm{H}$ NMR Spectrum of 22.
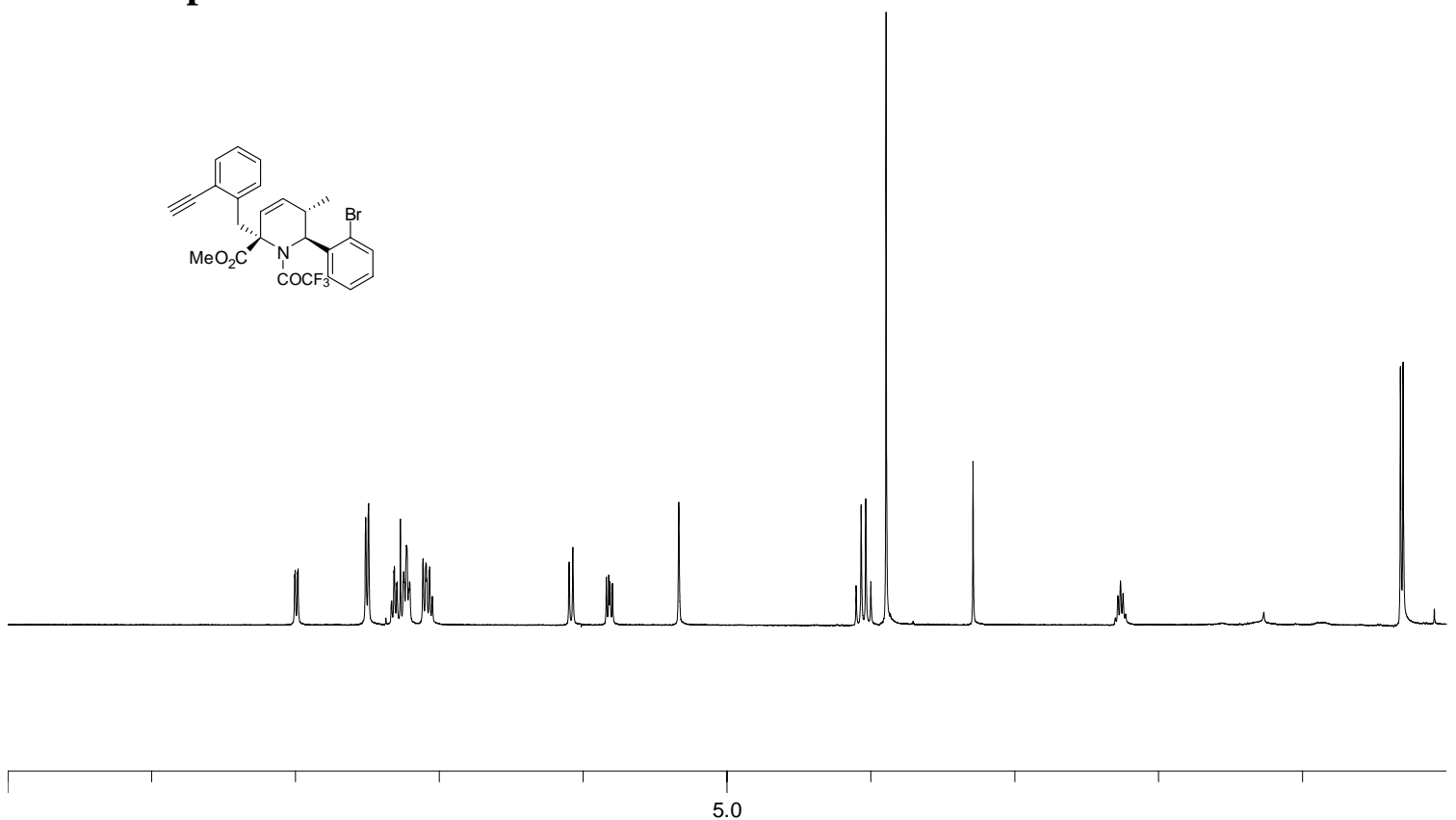

(2010

${ }^{13}$ C NMR Spectrum of 22.

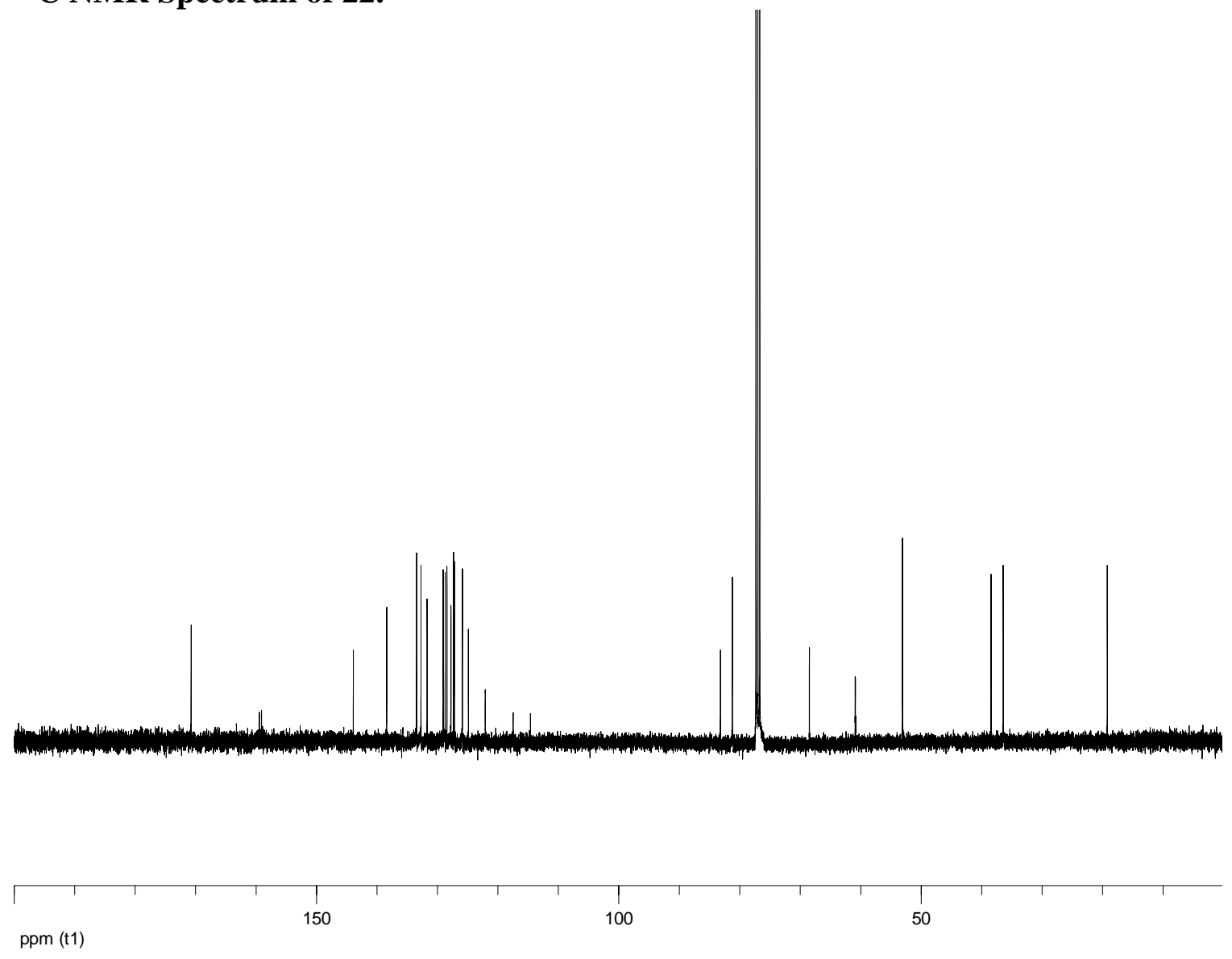

SI-16 
${ }^{1}$ H NMR Spectrum of 23.

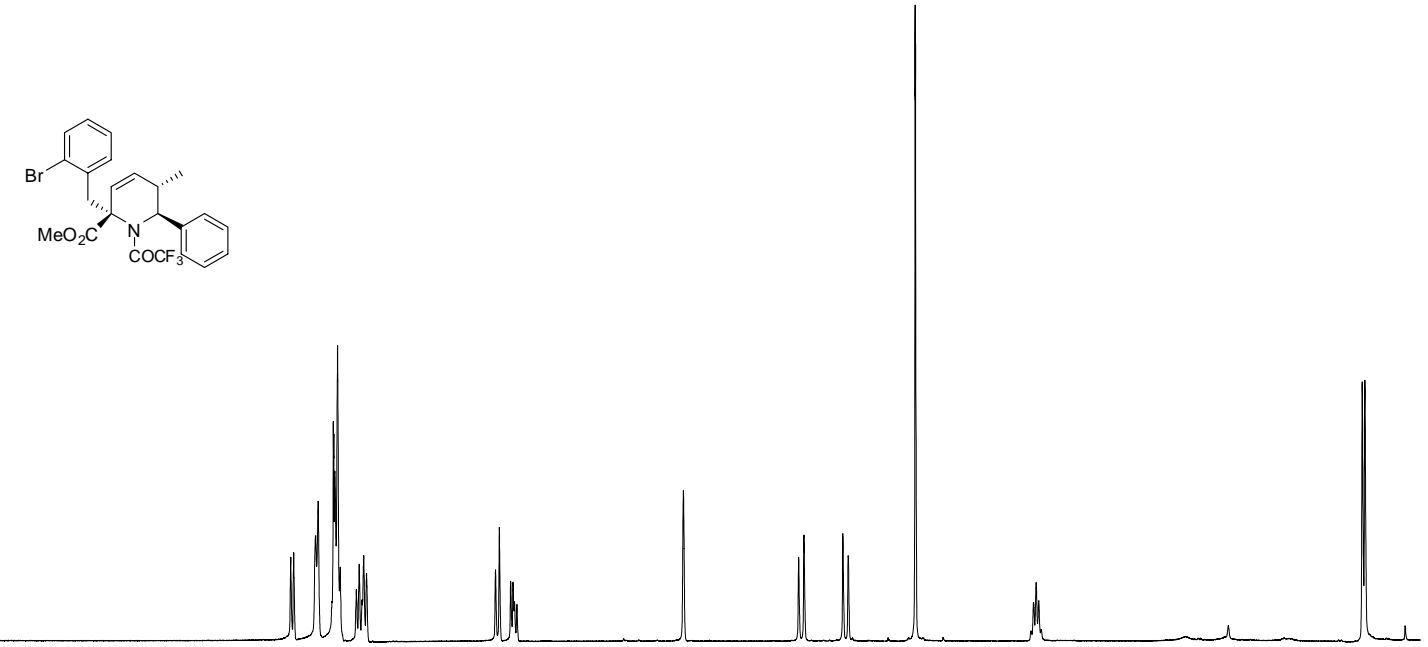

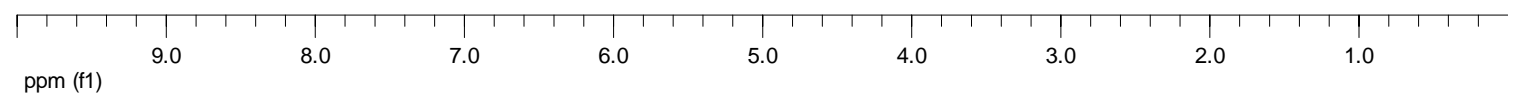

${ }^{13}$ C NMR Spectrum of 23.

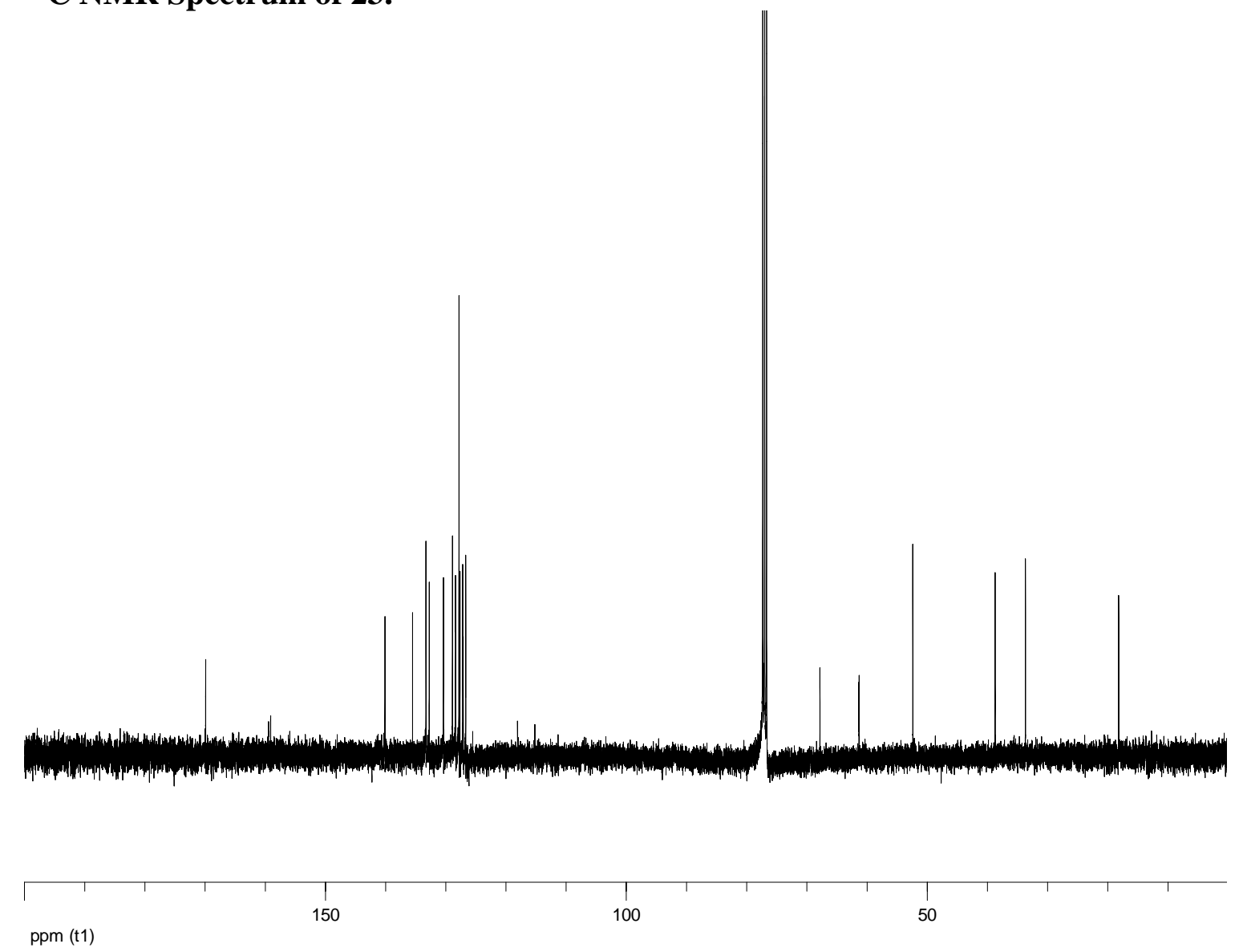


${ }^{1} \mathrm{H}$ NMR of 24.
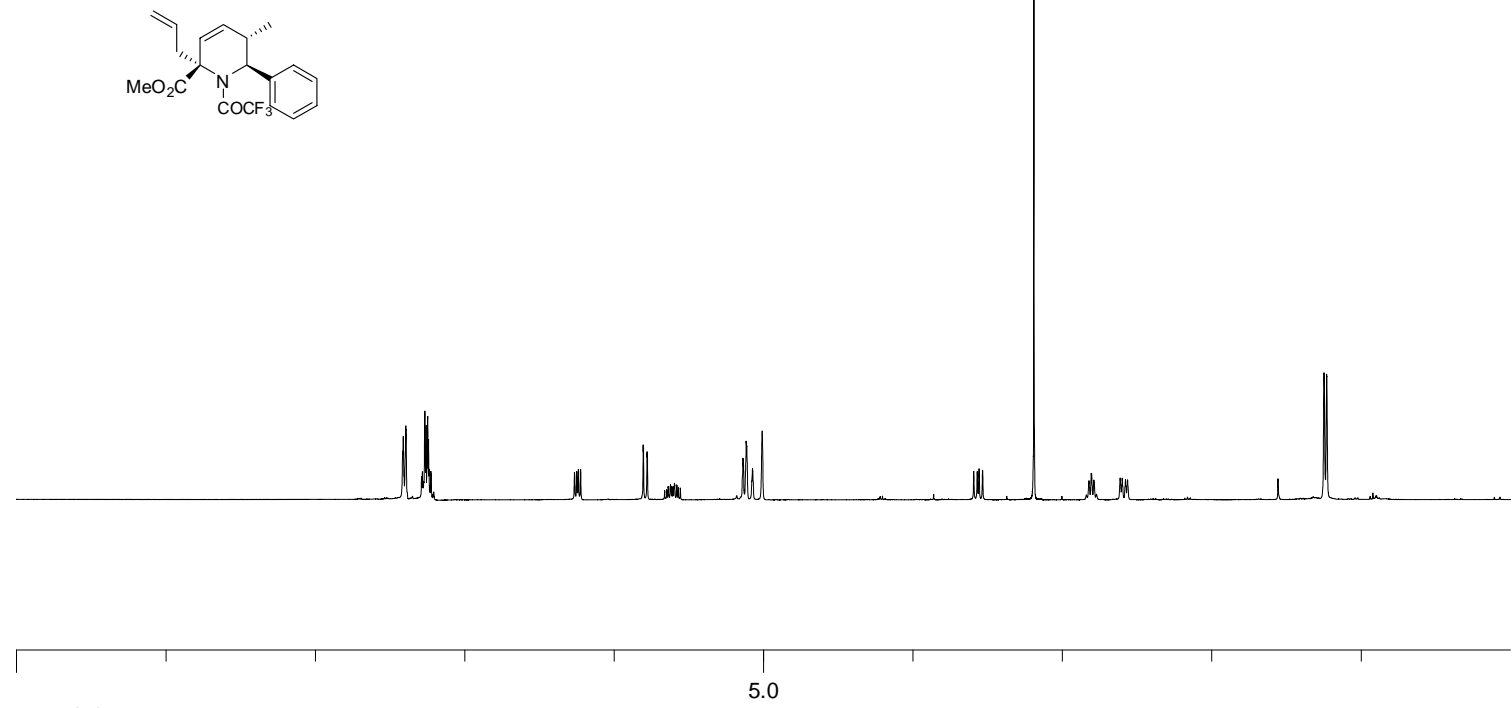

ppm (tI)

${ }^{13}$ C NMR of 24.
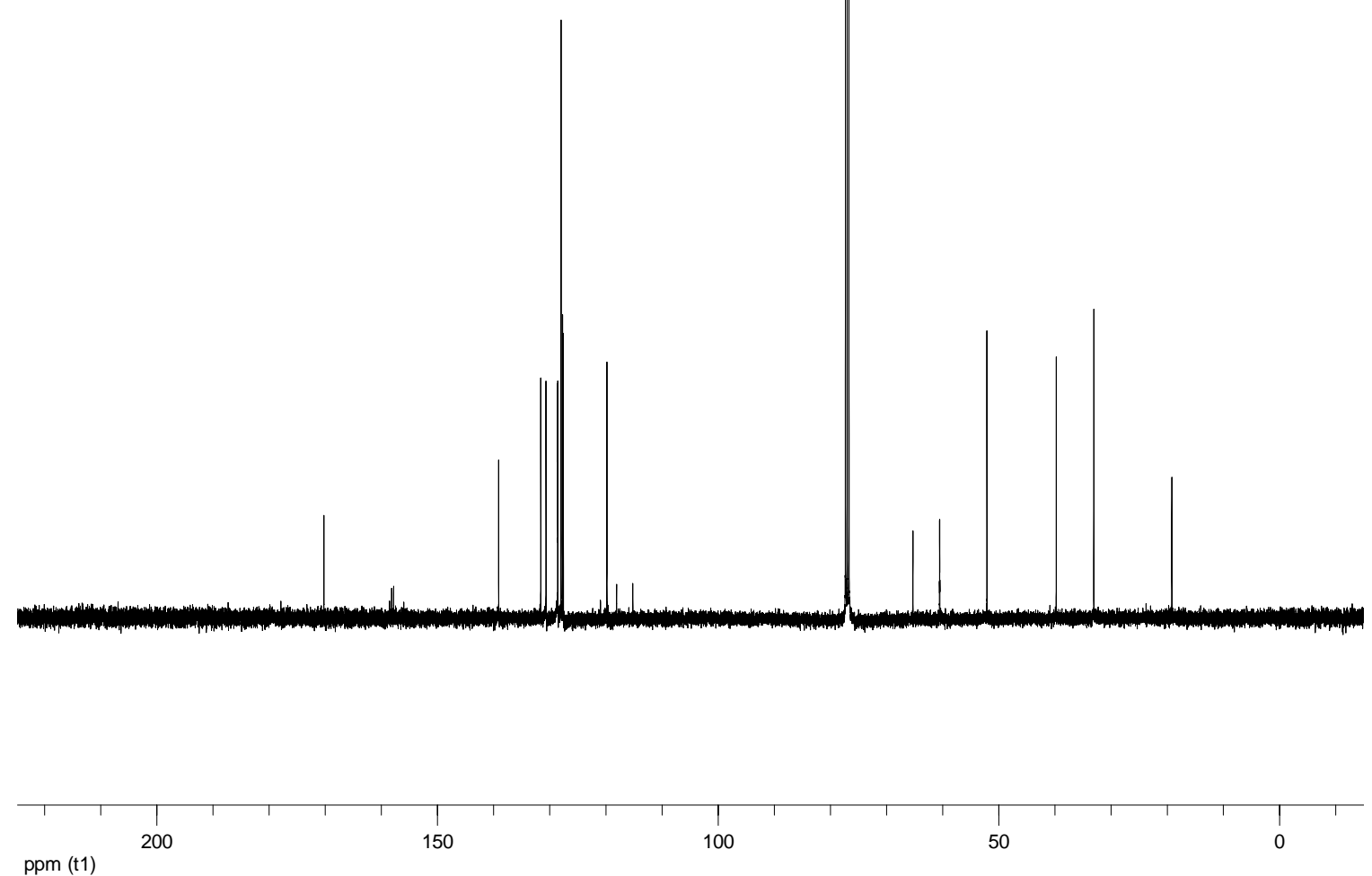

SI-18 


\section{Radical Cyclizations and Hydantoin formation}

General Procedure 2 - Radical cyclization of 23: A solution of $(2 R, 5 S, 6 S)-$ methyl 2-(2bromobenzyl)-5-methyl-6-phenyl-1-(2,2,2-trifluoroacetyl)-1,2,5,6-tetrahydropyridine-2carboxylate 23 (70 mg, $0.14 \mathrm{mmol}, 1$ equiv), 2,2'-azo-bis(isobutyronitrile) (2.3 mg, 0.014 mmol, 0.01 equiv.) and tri(n-butyl)tin hydride $(56 \mu \mathrm{L}, 0.21 \mathrm{mmol}, 1.5$ equiv.) in degassed benzene $(5 \mathrm{~mL})$ were heated at $80{ }^{\circ} \mathrm{C}$ for $4 \mathrm{~h}$. The reaction mixture was concentrated. Silica gel chromatography with ethyl acetate in hexane (from $0 \%$ to $10 \%$ ) gave the desired product $(2 S, 3 S, 4 \mathrm{a} S, 9 \mathrm{a} R)$-methyl 3-methyl-2-phenyl-1-(2,2,2-trifluoroacetyl)2,3,4,4a,9,9a-hexahydro-1H-indeno[2,1-b]pyridine-9a-carboxylate 25 as a viscous oil (48 $\mathrm{mg}, 81 \%$ yield).

(2S,3S,4aS,9aR)-Methyl 3-methyl-2-phenyl-1-(2,2,2-trifluoroacetyl)-2,3,4,4a,9,9a-

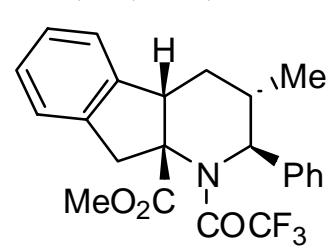
hexahydro-1H-indeno[2,1-b]pyridine-9a-carboxylate 25: This compound was synthesized by general procedure 2 and isolated in pure form as a colorless oil. ${ }^{1} \mathrm{H}$ NMR $\left(400 \mathrm{MHz}, \mathrm{CDCl}_{3}\right) \delta 7.73-$ $7.71(\mathrm{~m}, 2 \mathrm{H}), 7.44-7.40(\mathrm{~m}, 2 \mathrm{H}), 7.37-7.31(\mathrm{~m}, 1 \mathrm{H}), 7.26-7.22(\mathrm{~m}$, $4 \mathrm{H}), 4.75(\mathrm{~d}, J=8.0 \mathrm{~Hz}, 1 \mathrm{H}), 4.43(\mathrm{~d}, J=16.7 \mathrm{~Hz}, 1 \mathrm{H}), 3.64(\mathrm{~s}$, $3 \mathrm{H}), 3.60(\mathrm{dd}, J=12.5,5.0 \mathrm{~Hz}, 1 \mathrm{H}), 3.09(\mathrm{~d}, J=16.7 \mathrm{~Hz}, 1 \mathrm{H})$, 2.28-2.10 (m, 2H), 1.51-1.42 (m, $1 \mathrm{H}), 1.06(\mathrm{~d}, J=6.6 \mathrm{~Hz}, 3 \mathrm{H}) .{ }^{13} \mathrm{C}$ NMR $(100 \mathrm{MHz}$, $\left.\mathrm{CDCl}_{3}\right) \delta 173.7,160.8(\mathrm{q}, J=36.9 \mathrm{~Hz}), 143.1,141.5,138.9,128.5,128.2,127.8,127.6$, $126.9,124.2,123.1,116.2(\mathrm{q}, J=286.2 \mathrm{~Hz}), 72.1,66.2(\mathrm{q}, J=2.9 \mathrm{~Hz}), 52.5,49.7,43.1$, $36.5,35.4,19.8$. HRMS $\left(\mathrm{CI} / \mathrm{NH}_{3}\right) \mathrm{m} / \mathrm{z}$ calculated for $\left[\mathrm{C}_{22} \mathrm{H}_{22} \mathrm{NO}_{3} \mathrm{~F}_{3}+\mathrm{Na}\right] 440.1449$, found 440.1462. IR (thin film) $v_{\max } 2959,1760,1688,1192,1143 \mathrm{~cm}^{-1} \cdot[\alpha]_{\mathrm{D}}{ }^{20}+86.2^{\circ}(\mathrm{c}=0.8$, $\left.\mathrm{CHCl}_{3}\right)$.

(1R,3S,4R,6R)- $N$-(2,2,2-Trifluoroacetyl)-3-methoxycarbonyl-3,6-dimethyl-7,8-

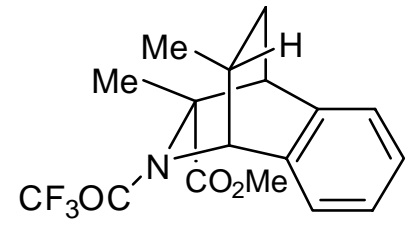
benzo-2-azabicyclo[2.2.2]octane 26: This compound was synthesized by general procedure 2 and isolated in pure form as a colorless oil. ${ }^{1} \mathrm{H}$ NMR $\left(400 \mathrm{MHz}, \mathrm{CDCl}_{3}\right) \delta 7.31-7.20(\mathrm{~m}$, $3 \mathrm{H}), 7.10-7.08(\mathrm{~m}, 1 \mathrm{H}), 4.90-4.88(\mathrm{~m}, 1 \mathrm{H}), 3.22(\mathrm{~s}, 3 \mathrm{H}), 3.16(\mathrm{t}$, $J=2.8 \mathrm{~Hz}, 1 \mathrm{H}), 1.92(\mathrm{~s}, 3 \mathrm{H}), 1.91-1.85(\mathrm{~m}, 1 \mathrm{H}), 1.82-1.75(\mathrm{~m}$, $2 \mathrm{H}), 1.25(\mathrm{~d}, J=6.8 \mathrm{~Hz}, 3 \mathrm{H}) .{ }^{13} \mathrm{C}$ NMR $\left(100 \mathrm{MHz}, \mathrm{CDCl}_{3}\right) \delta 171.2,156.32(\mathrm{q}, J=35.79$ $\mathrm{Hz}), 137.9,137.9,128.2,127.6,123.9,122.7,116.37$ (q, $J=288.3 \mathrm{~Hz}), 65.5,58.59$ (q, $J$ $=3.3 \mathrm{~Hz}), 52.1,45.2,32.1,26.7,20.0,18.5$. HRMS $\left(\mathrm{CI} / \mathrm{NH}_{3}\right) \mathrm{m} / \mathrm{z}$ calculated for $\left[\mathrm{C}_{17} \mathrm{H}_{18} \mathrm{NO}_{3} \mathrm{~F}_{3}+\mathrm{Na}\right]$ 364.1136, found 364.1163. $v_{\max } 2963,1733,1691,1201,1140 \mathrm{~cm}^{-1}$. $[\alpha]_{\mathrm{D}}{ }^{20}-62.3^{\circ}\left(\mathrm{c}=0.9, \mathrm{CHCl}_{3}\right)$.

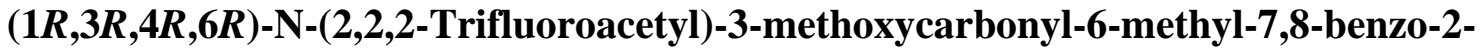

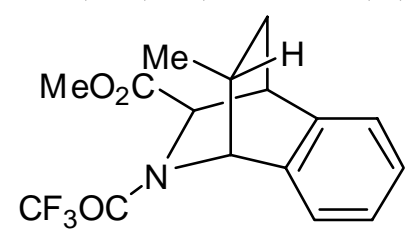
azabicyclo[2.2.2]octane 27: This compound was synthesized by general procedure 2 with the following exception. Tris (trimethylsilyl)silane was used in the place of tri(n-butyl)tin hydride. Compound 27 was isolated in the pure form as a viscous oil. ${ }^{1} \mathrm{H}$ NMR $\left(400 \mathrm{MHz}, \mathrm{CDCl}_{3}\right) \delta$ 7.35-7.25 (m, 3H), 7.22-7.20 (m, 1H), 4.91-4.89 (m, 1H), 4.11-4.08 (m, 1H), $3.84(\mathrm{~s}, 3 \mathrm{H}), 3.56(\mathrm{dd}, J=5.4$, $2.6 \mathrm{~Hz}, 1 \mathrm{H}), 1.92$ (dddd, $J=11.4,6.8,4.8,2.0 \mathrm{~Hz}, 1 \mathrm{H}), 1.79-1.72$ (m, 1H), 1.41-1.37 
(overlp m, 1H), 1.37 (d, $J=6.95 \mathrm{~Hz}, 3 \mathrm{H}) .{ }^{13} \mathrm{C}$ NMR $\left(100 \mathrm{MHz}, \mathrm{CDCl}_{3}\right) \delta 168.9,157.4$ $(\mathrm{q}, J=36.6 \mathrm{~Hz}), 139.5,138.9,128.6,127.8,124.5,122.7,116.52(\mathrm{q}, J=287.4 \mathrm{~Hz}), 61.0$, $58.73(\mathrm{q}, J=3.0 \mathrm{~Hz}), 52.7,37.6,33.2,27.7,18.0$. HRMS $\left(\mathrm{CI} / \mathrm{NH}_{3}\right) \mathrm{m} / \mathrm{z}$ calculated for $\left[\mathrm{C}_{16} \mathrm{H}_{16} \mathrm{NO}_{3} \mathrm{~F}_{3}+\mathrm{Na}\right]$ 350.0980, found 350.0994. $v_{\max } 2956,1757,1691,1206,1143 \mathrm{~cm}^{-1}$. $[\alpha]_{\mathrm{D}}^{20}+9.0^{\circ}\left(\mathrm{c}=1.2, \mathrm{CHCl}_{3}\right)$.

$(1 R, 3 S, 4 R, 6 R)-N-(2,2,2-$ Trifluoroacetyl)-3-methoxycarbonyl-6-methyl-7,8-benzo-2-

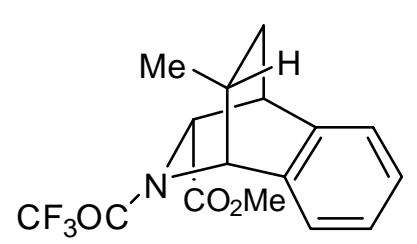
azabicyclo[2.2.2]octane 29: This compound was synthesized by general procedure 2 with the following exception. Tris (trimethylsilyl)silane was used in the place of tri(n-butyl)tin hydride. Compound 29 was isolated in the pure form as a viscous oil. ${ }^{1} \mathrm{H}$ NMR $\left(400 \mathrm{MHz}, \mathrm{CDCl}_{3}\right) \delta$ 7.30-7.21 (m, 3H), 7.15-7.13 (m, 1H), 4.92-4.90 (m, 1H), $4.52(\mathrm{~b} \mathrm{~m}, 1 \mathrm{H}), 3.53(\mathrm{dd}, J=5.6,2.5 \mathrm{~Hz}, 1 \mathrm{H})$, 3.44 (s, 3H), 2.03-1.86 (m, 2H), 1.37 (ddd, $J=12.5,4.4,2.3 \mathrm{~Hz}, 1 \mathrm{H}), 1.17$ (d, $J=6.67$ $\mathrm{Hz}, 3 \mathrm{H}) .{ }^{13} \mathrm{C}$ NMR $\left(100 \mathrm{MHz}, \mathrm{CDCl}_{3}\right) \delta 168.1,155.9(\mathrm{q}, J=36.6 \mathrm{~Hz}), 138.2,136.4$, $128.4,127.7,124.5,122.9,116.3(\mathrm{q}, J=285.6 \mathrm{~Hz}), 57.9$ (q, $J=3.0 \mathrm{~Hz}), 60.8,52.2,38.1$, 33.2, 31.7, 19.2. HRMS $\left(\mathrm{CI} / \mathrm{NH}_{3}\right) \mathrm{m} / \mathrm{z}$ calculated for $\left[\mathrm{C}_{16} \mathrm{H}_{16} \mathrm{NO}_{3} \mathrm{~F}_{3}+\mathrm{Na}\right] 350.0980$, found 350.1003. $v_{\max } 2959,1760,1689,1192,1143 \mathrm{~cm}^{-1} .[\alpha]_{\mathrm{D}}{ }^{20}-41.2^{\circ}\left(\mathrm{c}=0.9, \mathrm{CHCl}_{3}\right)$.

Hydantoin 35: A solution of potassium hydroxide in water $(3.3 \mathrm{M}, 2 \mathrm{~mL})$ was added to a

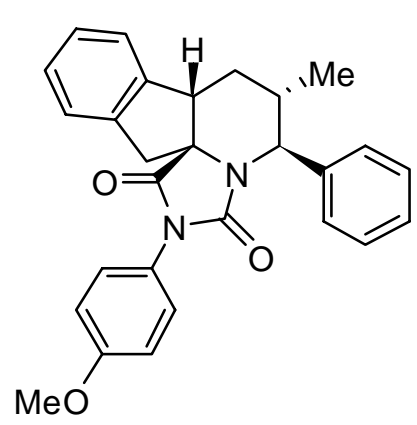
solution of $(2 S, 3 S, 4 \mathrm{a} S, 9 \mathrm{a} R)$-methyl 3-methyl-2-phenyl-1(2,2,2-trifluoroacetyl)-2,3,4,4a,9,9a-hexahydro-1H-indeno[2,1b]pyridine-9a-carboxylate 25 ( $10 \mathrm{mg}, 0.024 \mathrm{mmol}, 1$ equiv.) in methanol $(3 \mathrm{~mL})$ and stirred at room temperature for $2 \mathrm{~h}$. A solution of aqueous hydrochloric acid $(6 \mathrm{M}, 0.3 \mathrm{~mL})$ was added and the reaction mixture was concentrated. 4Methoxyphenylisocyanate ( $5 \mu \mathrm{L}, 0.04 \mathrm{mmol}, 1.7$ equiv.), triethylamine $(10 \mu \mathrm{L}, 0.07 \mathrm{mmol}, 3$ equiv) and THF $(1 \mathrm{~mL})$ were added and the reaction mixture was heated at $45^{\circ} \mathrm{C}$ for 6

h. The reaction was quenched by the addition of water $(2 \mathrm{~mL})$ and the crude reaction mixture was extracted with dichloromethane $(3 \times 10 \mathrm{~mL})$. The combined organic layers were dried with sodium sulfate, concentrated. Silica gel chromatography with ethyl acetate in hexane (gradient elution from $0 \%$ to $50 \%$ ) gave the desired product $35(5 \mathrm{mg}$, $68 \%$ yield) as a colorless oil. ${ }^{1} \mathrm{H}$ NMR $\left(400 \mathrm{MHz}, \mathrm{CDCl}_{3}\right) \delta$ 7.45-7.19 $(\mathrm{m}, 11 \mathrm{H}), 6.89-$ $6.83(\mathrm{~m}, 2 \mathrm{H}), 3.90(\mathrm{~d}, J=11.1 \mathrm{~Hz}, 1 \mathrm{H}), 3.76(\mathrm{~s}, 3 \mathrm{H}), 3.67(\mathrm{dd}, J=7.9,5.8 \mathrm{~Hz}, 1 \mathrm{H})$, 3.63-3.59 (d, $2 \mathrm{H}), 2.72-2.59(\mathrm{~m}, 1 \mathrm{H}), 2.29(\mathrm{ddd}, J=14.9,5.9,5.9 \mathrm{~Hz}, 1 \mathrm{H}), 1.81$ (ddd, $J$ $=15.0,7.6,7.6 \mathrm{~Hz}, 1 \mathrm{H}), 0.78(\mathrm{~d}, J=6.71 \mathrm{~Hz}, 3 \mathrm{H}) .{ }^{13} \mathrm{C}$ NMR $\left(100 \mathrm{MHz}, \mathrm{CD}_{3} \mathrm{CN}\right)$ $\delta 174.6,158.9,153.8,144.1,138.8,135.6,129.5,128.0,127.9,127.6,127.5,127.4$, $124.8,124.3,123.8,114.0,70.7,62.1,55.4,46.7,39.1,35.4,31.6,19.5$. HRMS (CI/NH $\left.{ }_{3}\right)$ $\mathrm{m} / \mathrm{z}$ calculated for $\left[\mathrm{C}_{28} \mathrm{H}_{27} \mathrm{~N}_{2} \mathrm{O}_{3} \mathrm{~F}_{3}\right]$ 439.2022, found 439.2027. $v_{\max } 2926,1770,1721$, $1514,1411,1250,1173 \mathrm{~cm}^{-1} .[\alpha]_{\mathrm{D}}^{20}-39.4^{\circ}\left(\mathrm{c}=0.6, \mathrm{CHCl}_{3}\right)$. 
Compound 36. This compound was synthesized by general procedure 2 with the

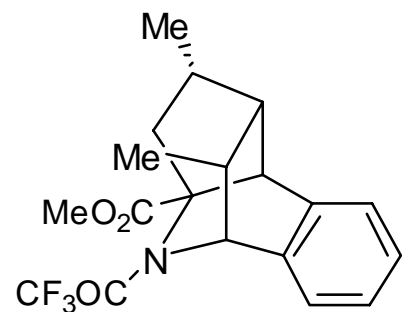
following exception. Tris(trimethylsilyl)silane was used in the place of tri(n-butyl)tin hydride. Compound $\mathbf{3 6}$ was isolated in the pure form as a viscous oil. Viscous oil. ${ }^{1} \mathrm{H}$ NMR $(400 \mathrm{MHz}$, $\left.\mathrm{C}_{6} \mathrm{D}_{6}\right) \delta$ 7.07-7.03 (m, 2H), 6.95-6.91 (m, 1H), 6.90-6.88 (m, $1 \mathrm{H}), 4.53(\mathrm{~b}, 1 \mathrm{H}), 3.39(\mathrm{~d}, J=3.9 \mathrm{~Hz}, 1 \mathrm{H}), 3.00(\mathrm{~s}, 3 \mathrm{H}), 2.55-$ $2.45(\mathrm{~m}, 2 \mathrm{H}), 1.93-1.84(\mathrm{~m}, 1 \mathrm{H}), 1.41-1.33(\mathrm{~m}, 2 \mathrm{H}), 0.90(\mathrm{~d}, J$ $=7.1 \mathrm{~Hz}, 3 \mathrm{H}), 0.67(\mathrm{~d}, J=6.1 \mathrm{~Hz}, 3 \mathrm{H}) .{ }^{13} \mathrm{C}$ NMR $(100 \mathrm{MHz}$, $\left.\mathrm{CDCl}_{3}\right) \delta 169.8,155.1(\mathrm{q}, J=36.1 \mathrm{~Hz}), 139.4,133.7,128.1$, $127.4,125.8,121.8,116.3(\mathrm{q}, J=286.1 \mathrm{~Hz}), 71.7,58.1$ (q, $J=3.0 \mathrm{~Hz}), 52.2,49.2,45.1$, $39.8,37.9,29.5,23.4,14.7$. HRMS $(\mathrm{CI} / \mathrm{NH} 3) \mathrm{m} / \mathrm{z}$ calculated for $\left[\mathrm{C}_{19} \mathrm{H}_{20} \mathrm{NO}_{3} \mathrm{~F}_{3}+\mathrm{Na}\right]$ 320.1293 , found 320.1299. $v_{\max } 2962,1739,1684,1203,1143 \mathrm{~cm}^{-1} \cdot[\alpha]_{\mathrm{D}}{ }^{20}+21.0^{\circ}(\mathrm{c}=$ $\left.1.0, \mathrm{CHCl}_{3}\right)$.

Compound 37. This compound was synthesized by general procedure 2 and isolated in

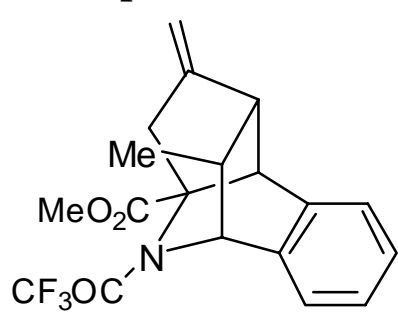
pure form as a colorless oil. ${ }^{1} \mathrm{H}$ NMR $\left(400 \mathrm{MHz}, \mathrm{CDCl}_{3}\right) \delta$ 7.37$7.31(\mathrm{~m}, 2 \mathrm{H}), 7.30-7.23(\mathrm{~m}, 1 \mathrm{H}), 7.22-7.17(\mathrm{~m}, 1 \mathrm{H}), 5.11$ (app t, $J$ $=2.4 \mathrm{~Hz}, 1 \mathrm{H}), 5.07(\mathrm{app} \mathrm{t}, J=2.4 \mathrm{~Hz}, 1 \mathrm{H}), 4.77(\mathrm{~b} \mathrm{~s}, 1 \mathrm{H}), 3.51$ $(\mathrm{d}, J=4.7 \mathrm{~Hz}, 1 \mathrm{H}), 3.45(\mathrm{ddd}, J=18.3,2.4,2.4 \mathrm{~Hz}, 1 \mathrm{H}), 3.36$ (s, 3H), 2.87 (ddd, $J=18.3,2.4,2.4 \mathrm{~Hz}, 1 \mathrm{H}), 2.77(\mathrm{dd}, J=9.8,4.7$ $\mathrm{Hz}, 1 \mathrm{H}), 1.95-1.87(\mathrm{~m}, 1 \mathrm{H}), 1.02(\mathrm{~d}, J=7.21 \mathrm{~Hz}, 3 \mathrm{H}) .{ }^{13} \mathrm{C}$ NMR $\left(75 \mathrm{MHz}, \mathrm{CDCl}_{3}\right) \delta .169 .2,155.33(\mathrm{q}, J=36.47 \mathrm{~Hz}), 145.8$, $139.1,133.3,128.3,127.7,125.8,121.9,116.20(\mathrm{q}, J=287.8 \mathrm{~Hz}), 110.9,70.6,58.01$ (q, $J=3.2 \mathrm{~Hz}), 52.3,51.1,45.3,38.7,37.8,15.5$. HRMS $(\mathrm{CI} / \mathrm{NH} 3) \mathrm{m} / \mathrm{z}$ calculated for $\left[\mathrm{C}_{19} \mathrm{H}_{18} \mathrm{NO}_{3} \mathrm{~F}_{3}+\mathrm{Na}\right]$ 388.1136, found 388.1183. $v_{\max } 2962,1739,1684,1203,1143 \mathrm{~cm}^{-1}$. $[\alpha]_{\mathrm{D}}^{20}+31.0^{\circ}\left(\mathrm{c}=1.0, \mathrm{CHCl}_{3}\right)$. 
5. ${ }^{1} \mathrm{H},{ }^{13} \mathrm{C}$ and 2D NMR Spectra of Products of Radical Cyclizations and Hydantoin 35.

${ }^{1} \mathrm{H}$ NMR of 25.
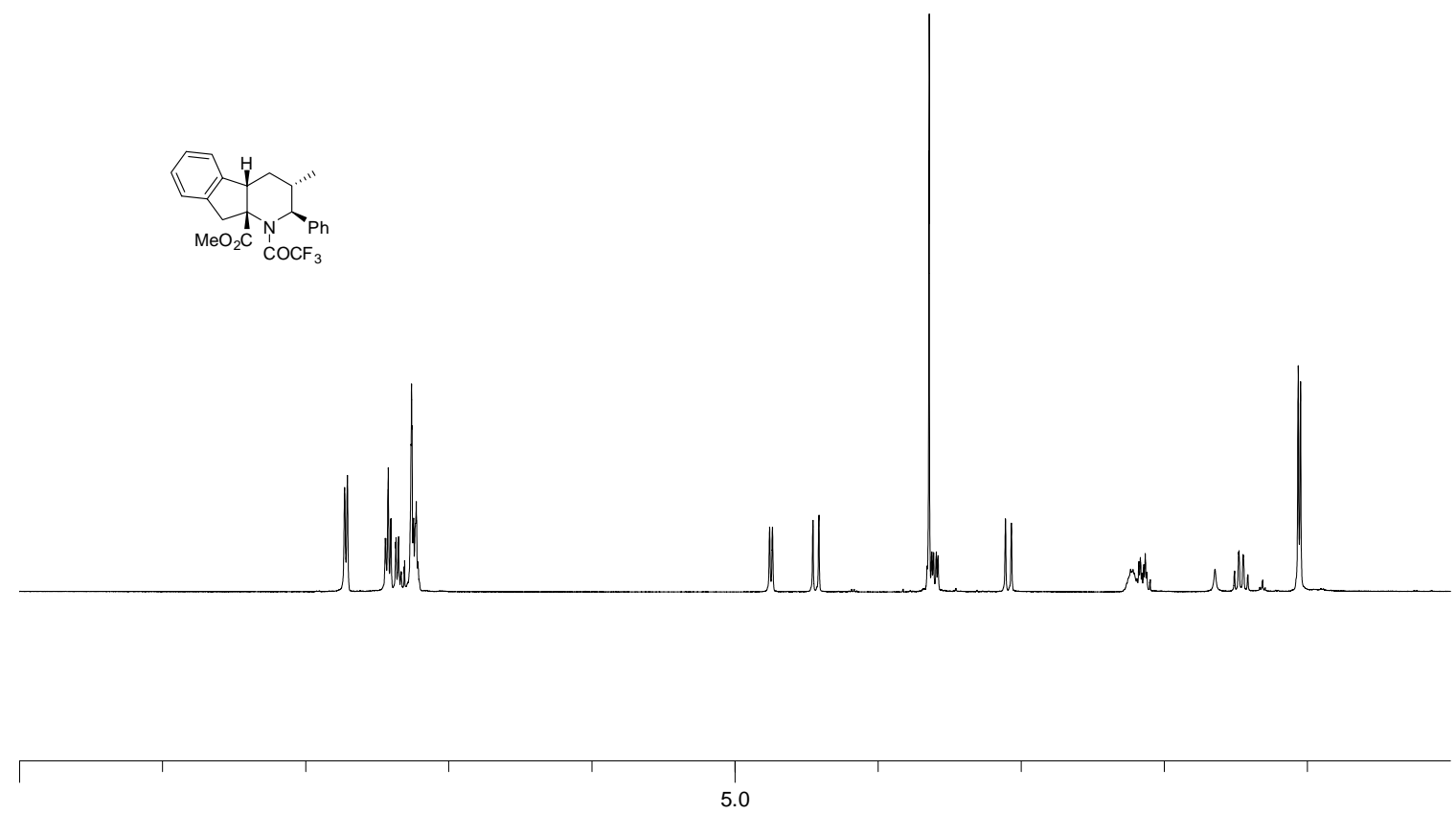

ppm (t1)

${ }^{13} \mathrm{C}$ NMR Spectrum of 25.

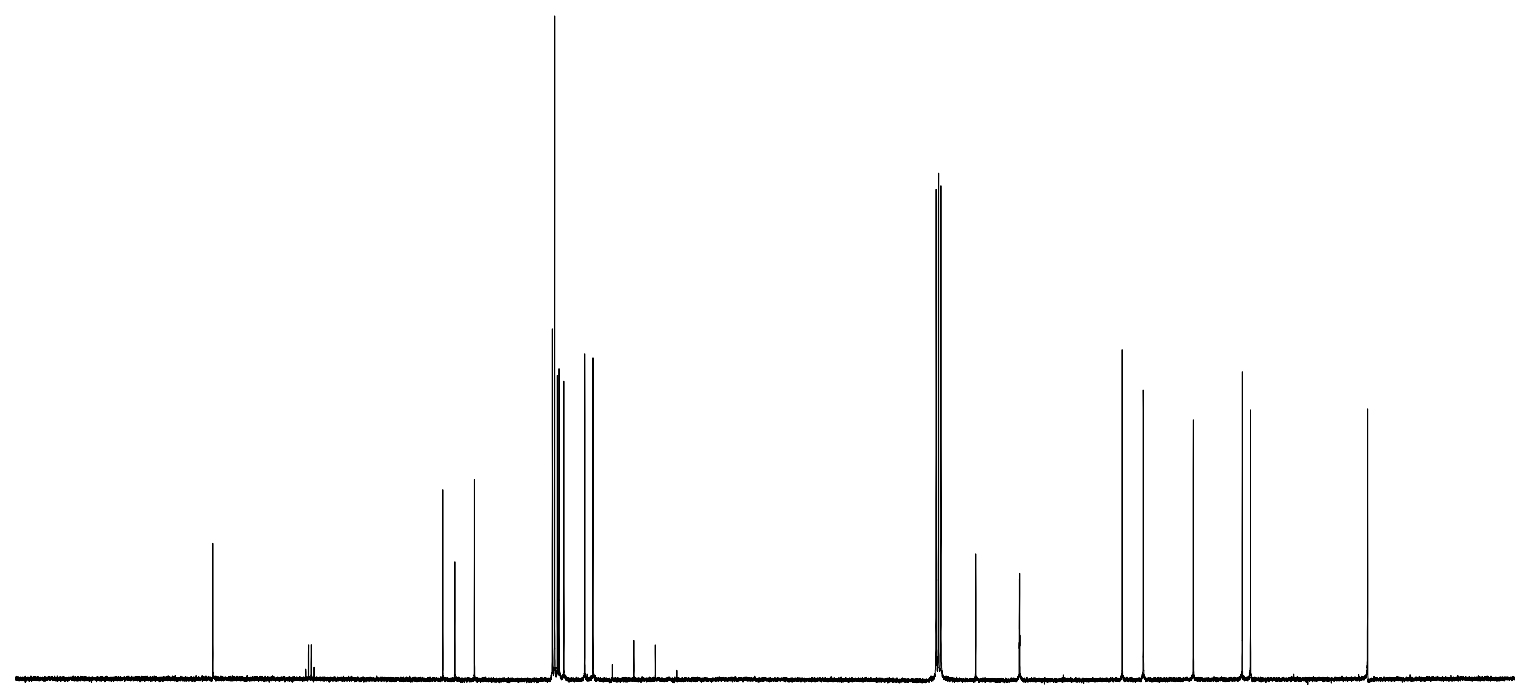


${ }^{1} \mathrm{H}$ NMR Spectrum of 26.

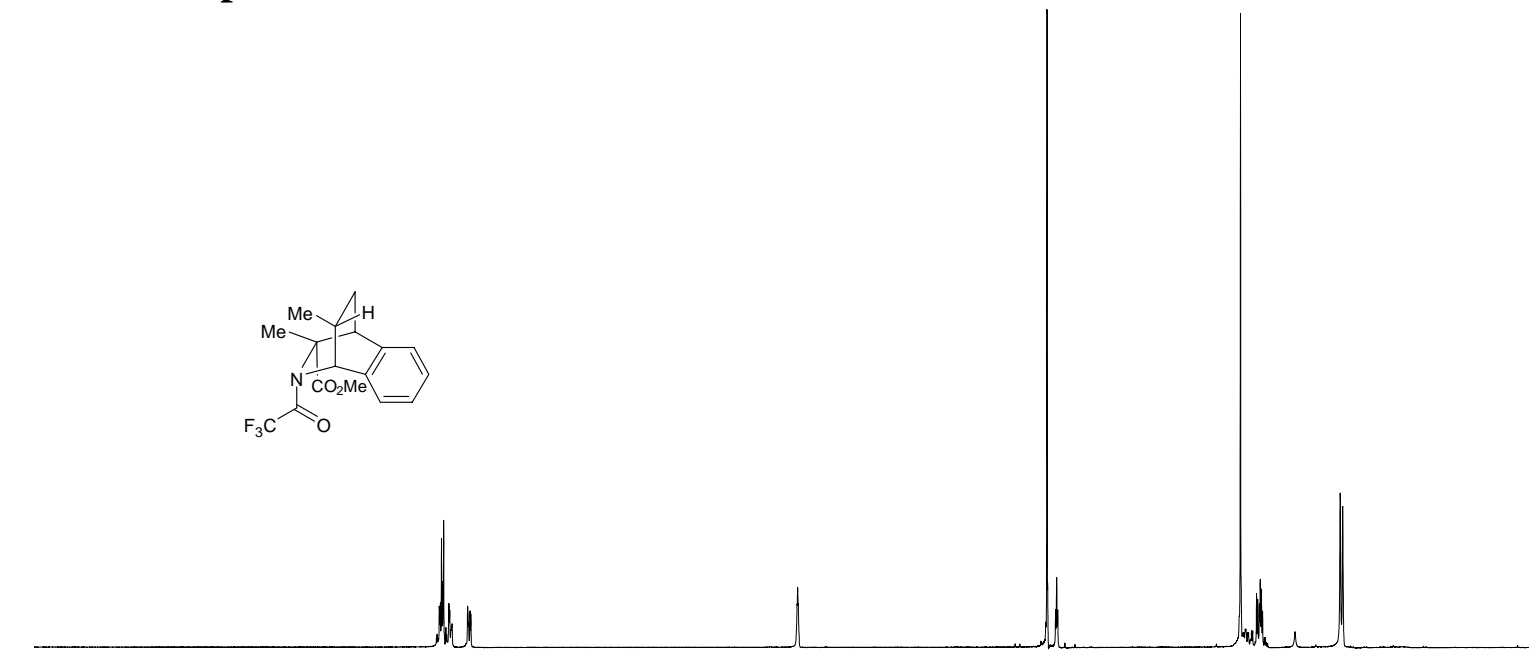

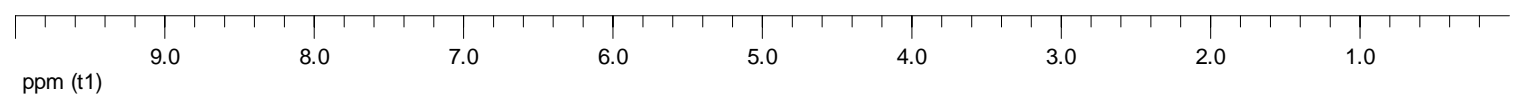

${ }^{13}$ C NMR Spectrum of 26.

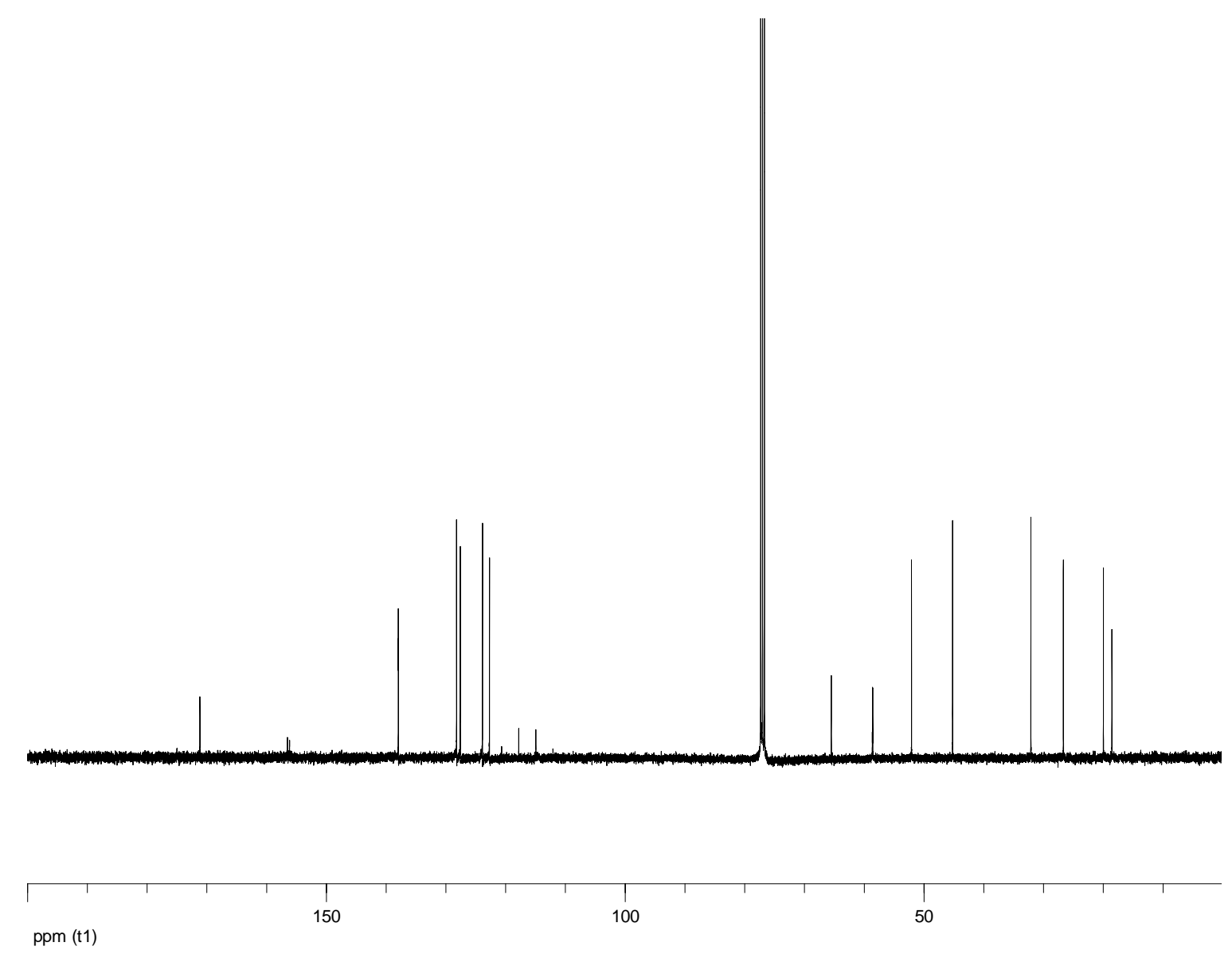




\section{Expanded Segment of HMBC Spectrum of 26.}

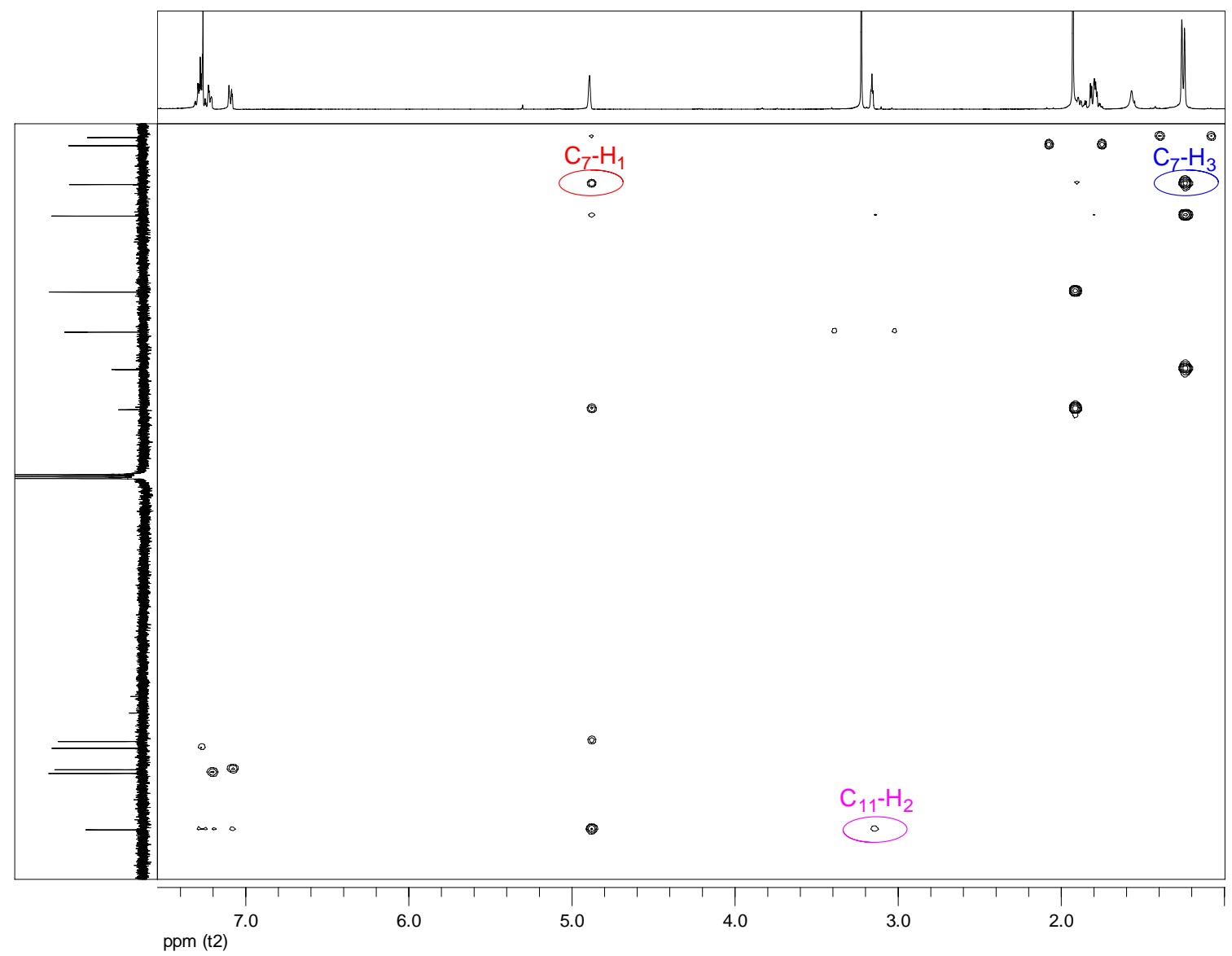

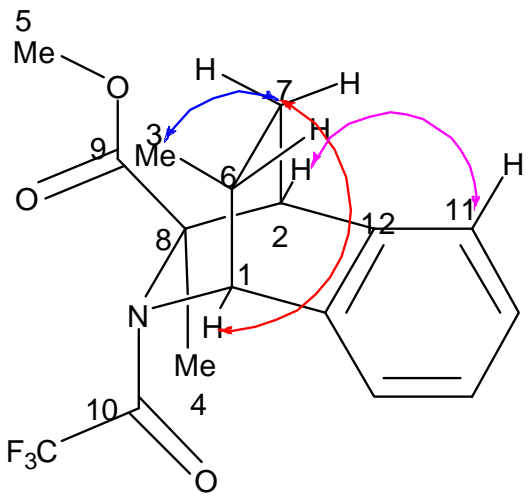

$C_{11}-H_{2}$ cross peak provides evidence for the $C_{2}-C_{12}$ bond formed in this reaction

The strong three-bond HMBC cross peaks across $\mathrm{C}_{7}-\mathrm{H}_{3}$ and $\mathrm{C}_{7}-\mathrm{H}_{1}$ provide evidence for the $[2,2,2]-$ bicyclo product as opposed to the [3,2,1]-bicyclo product $A$ shown on the right.

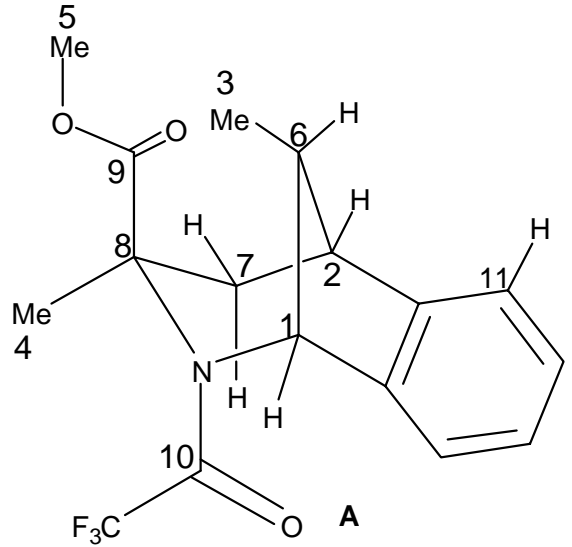

(This compound is not formed)

$\mathrm{C}_{7}-\mathrm{H}_{3}$ and $\mathrm{C}_{7}-\mathrm{H}_{1}$ are four bonds away in this structure and will not show (or show extremely weak) HMBC crosspeaks. The observed strong HMBC cross peaks for these bonds discount this structure. 


\section{${ }^{1}$ H NMR Spectrum of 27.}
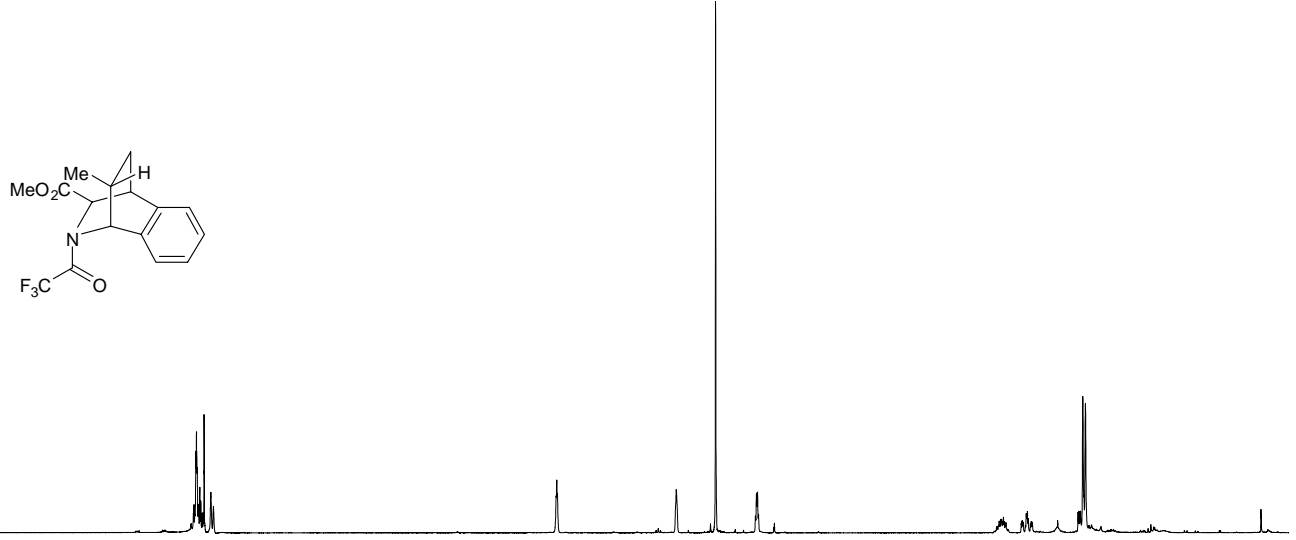

ppm (t1)

${ }^{13}$ C NMR Spectrum of 27.

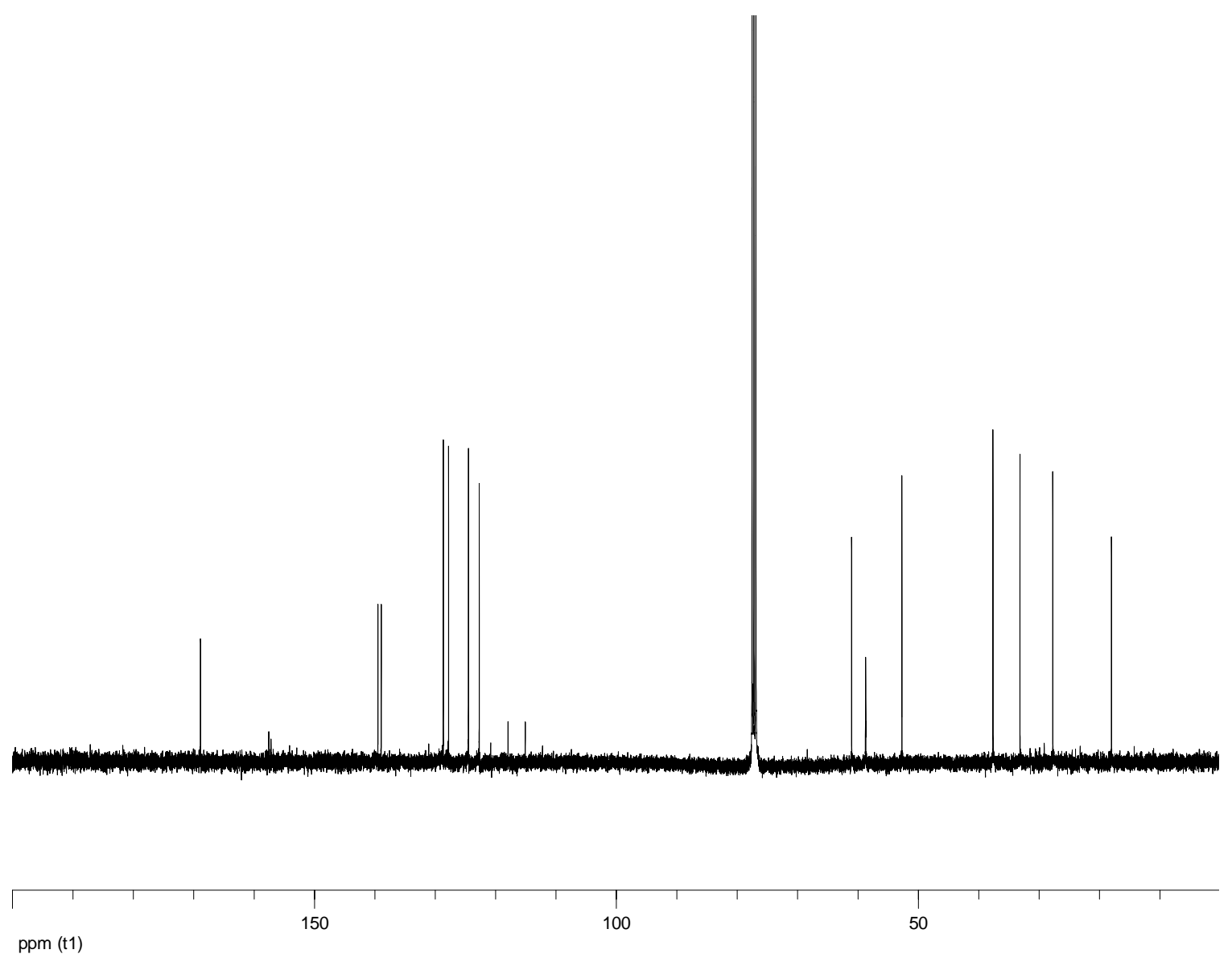


${ }^{1}$ H NMR Spectrum of 29.
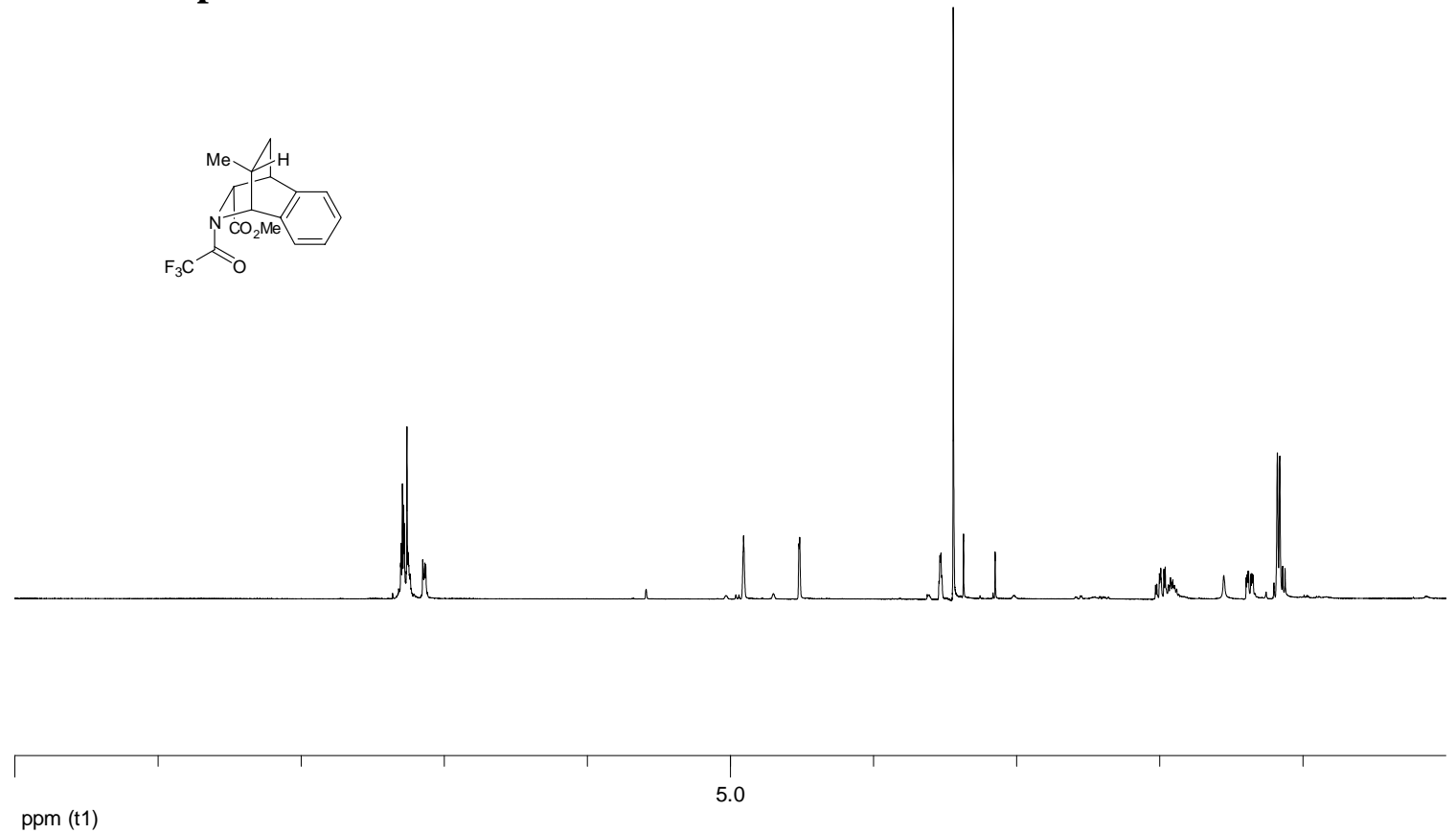

ppm (t1)

\section{${ }^{13}$ C NMR Spectrum of 29.}
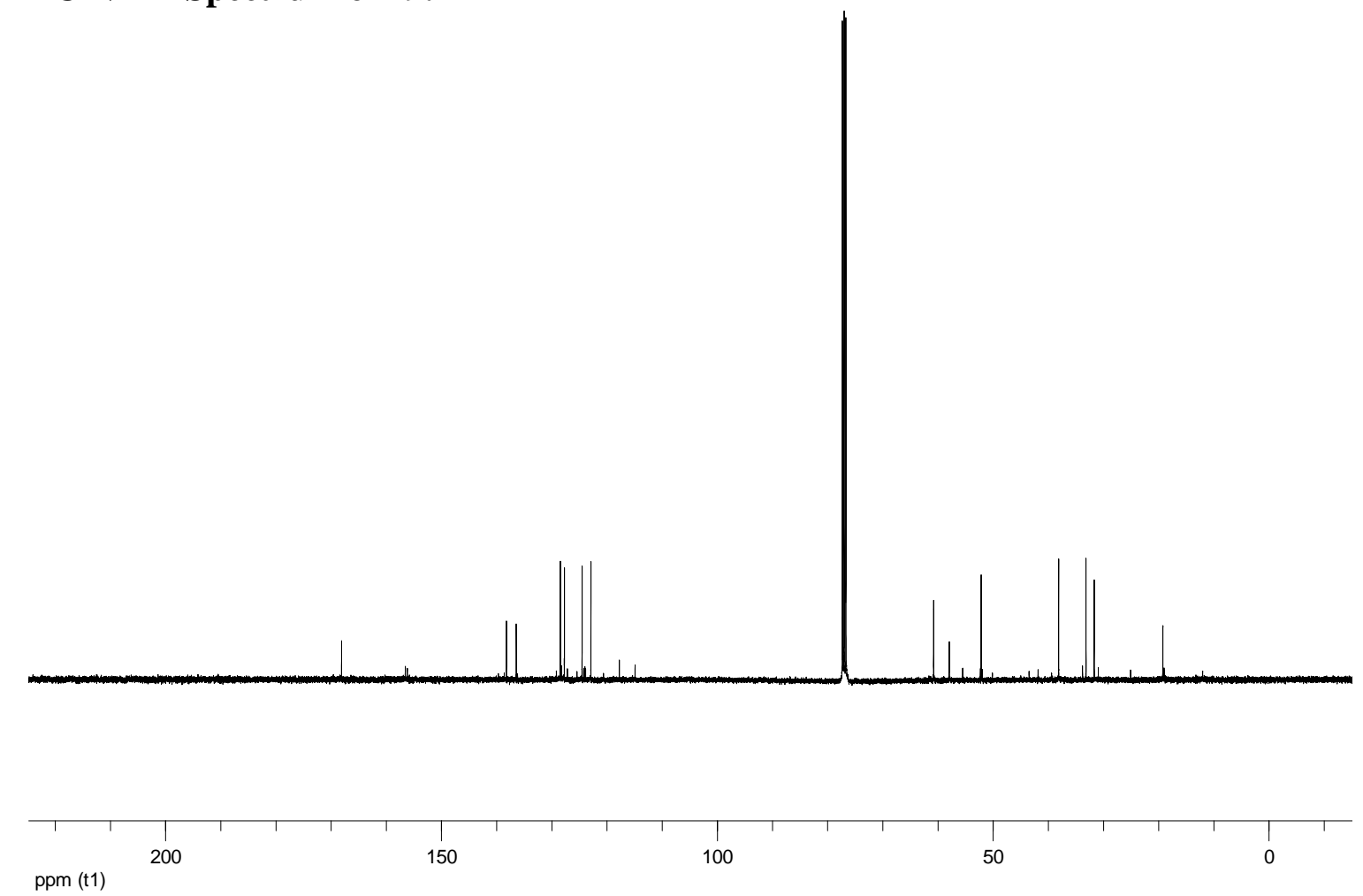
${ }^{1}$ H NMR Spectrum of 35.

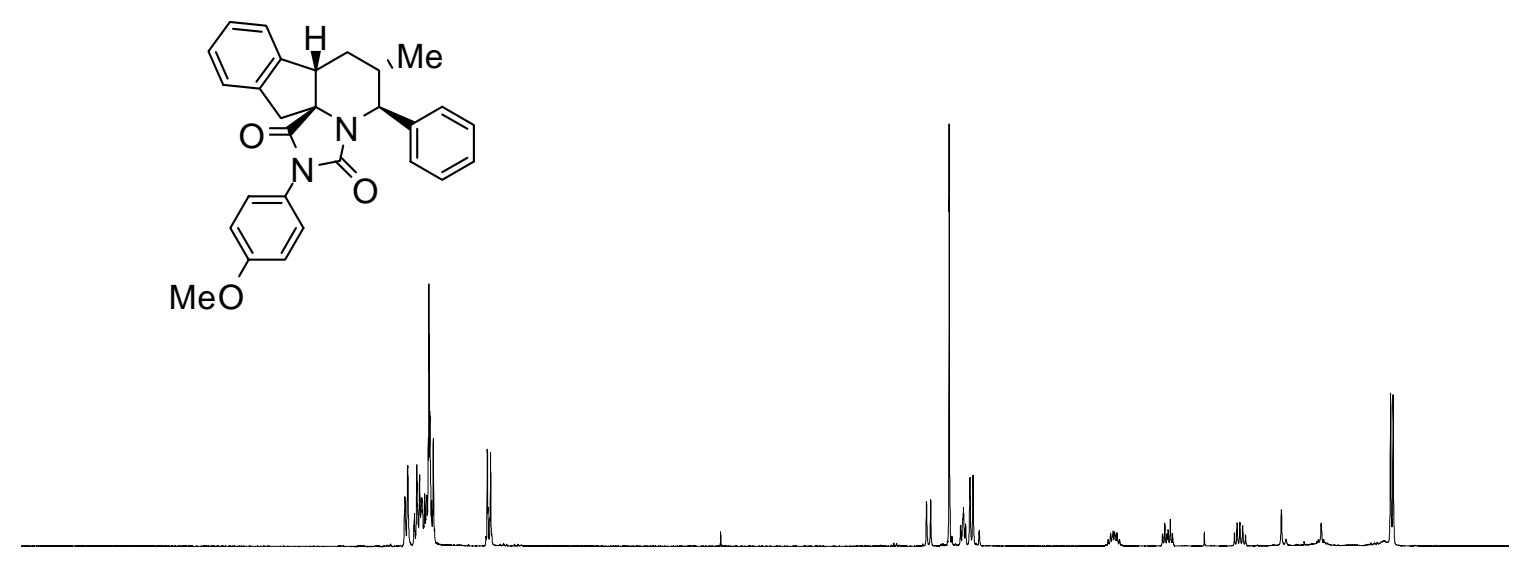

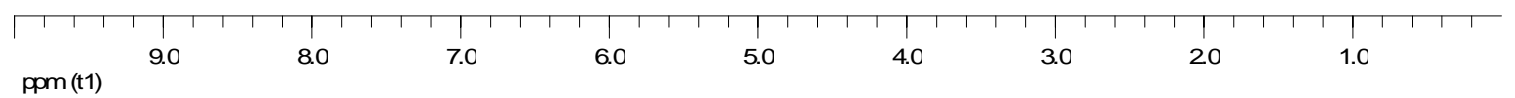

${ }^{13}$ C NMR Spectrum of 35.

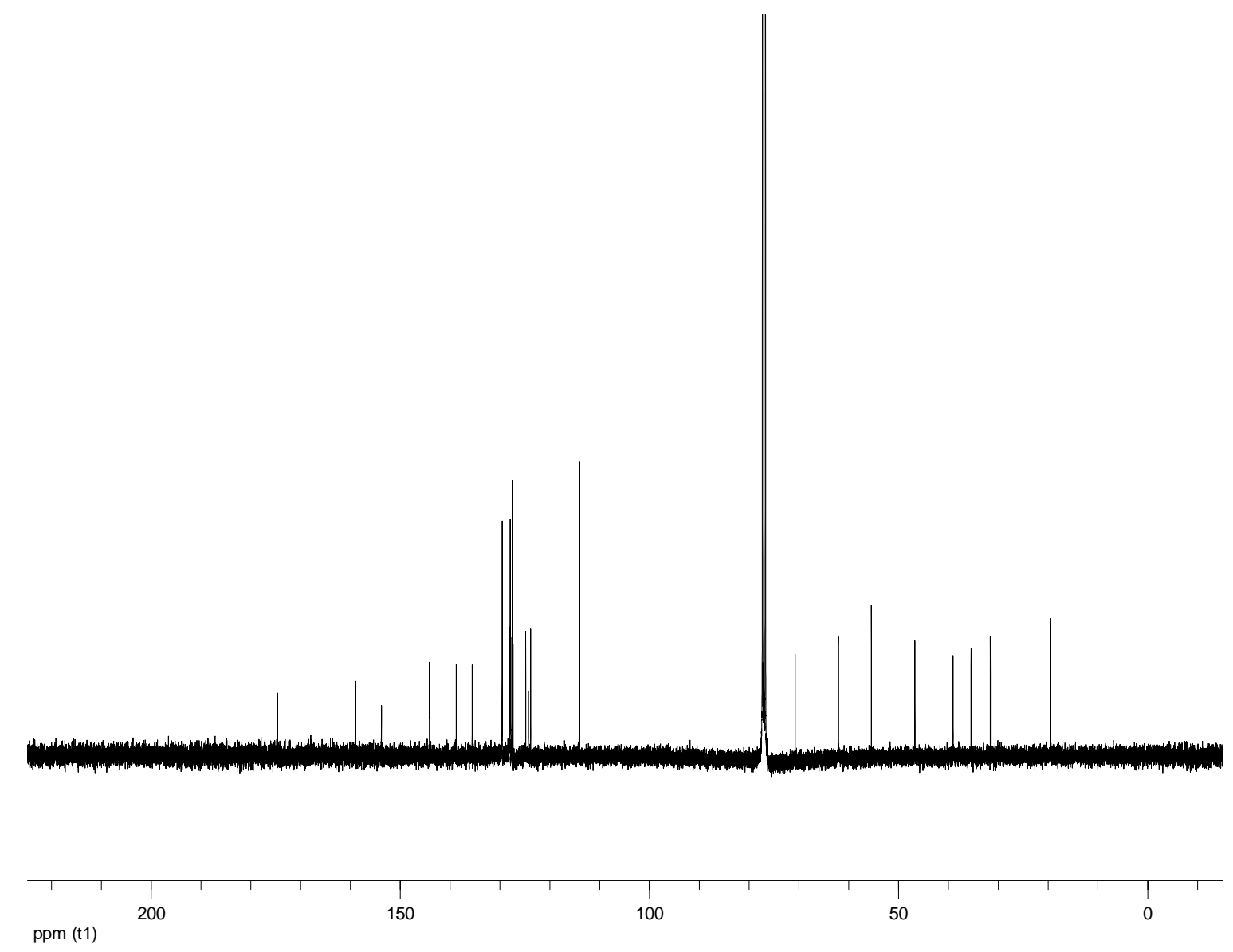


${ }^{1} \mathbf{H}$ NMR Spectrum of 36. (in benzene-d6)
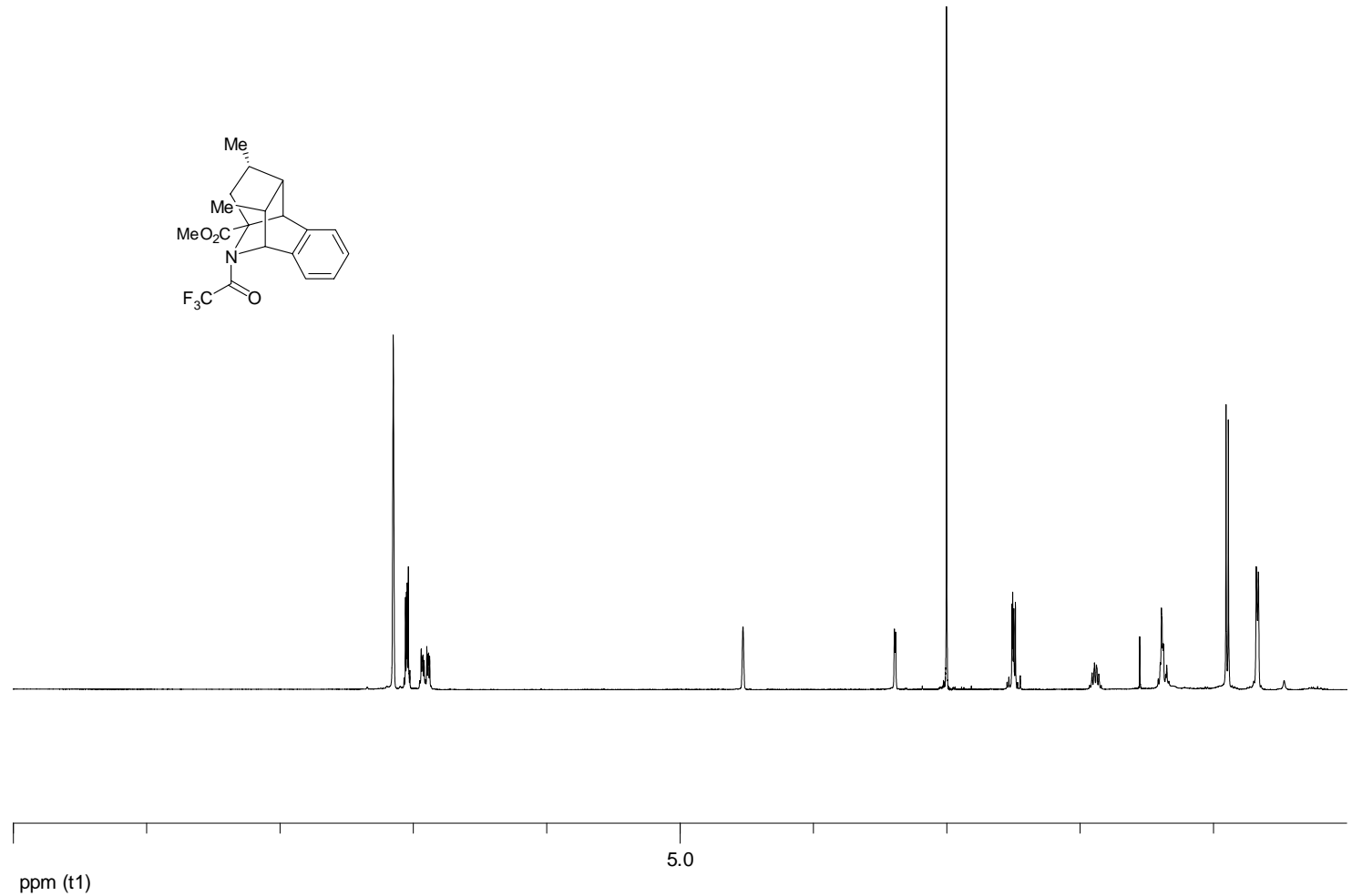

${ }^{13} \mathrm{C}$ NMR Spectrum of 36. (in $\mathrm{CDCl}_{3}$ )
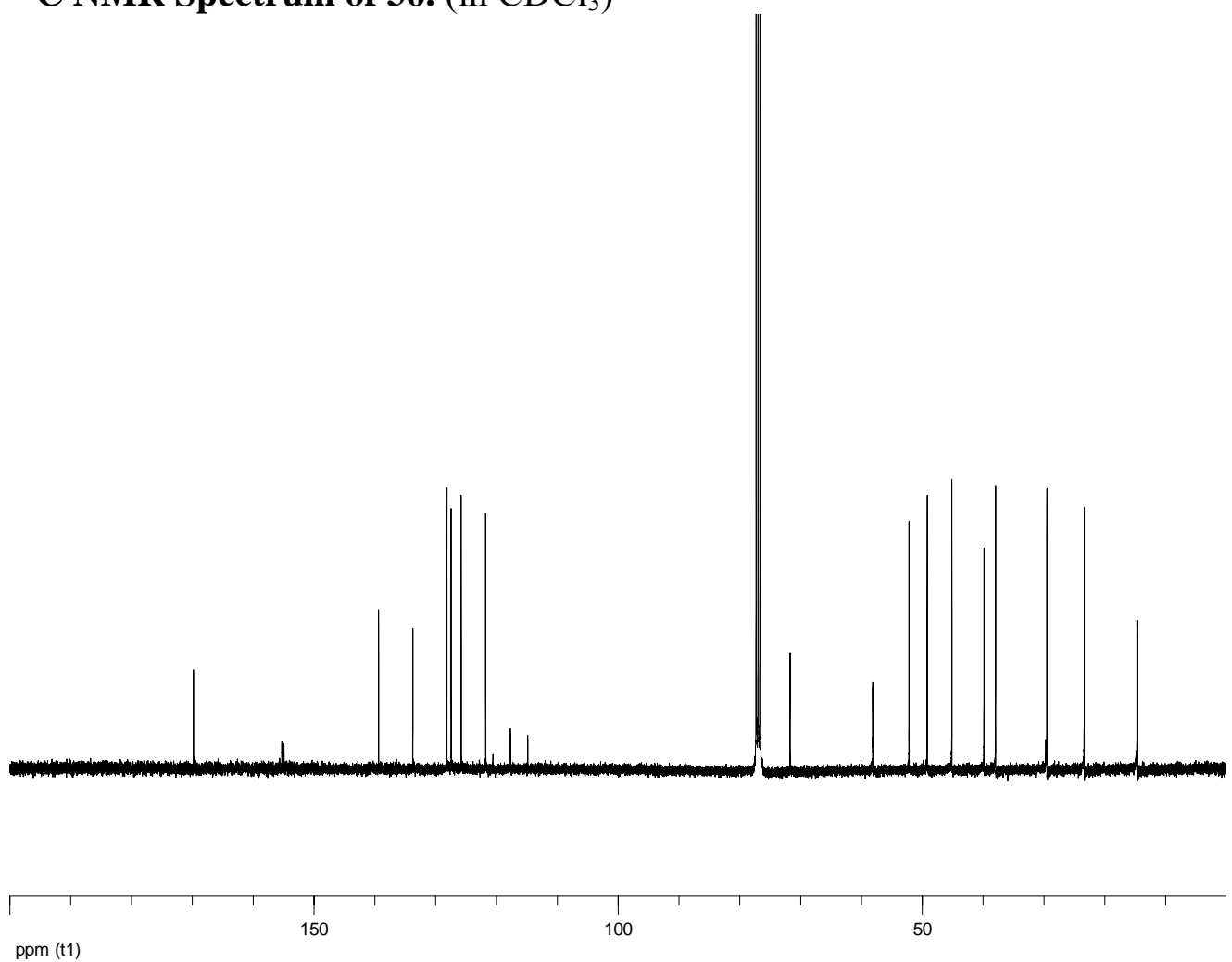
Expanded HMBC Spectrum of $36\left({ }^{1} \mathrm{H},{ }^{13} \mathrm{C}\right.$ and $\mathrm{HMBC}$ spectra were obtained in benzene-d6).
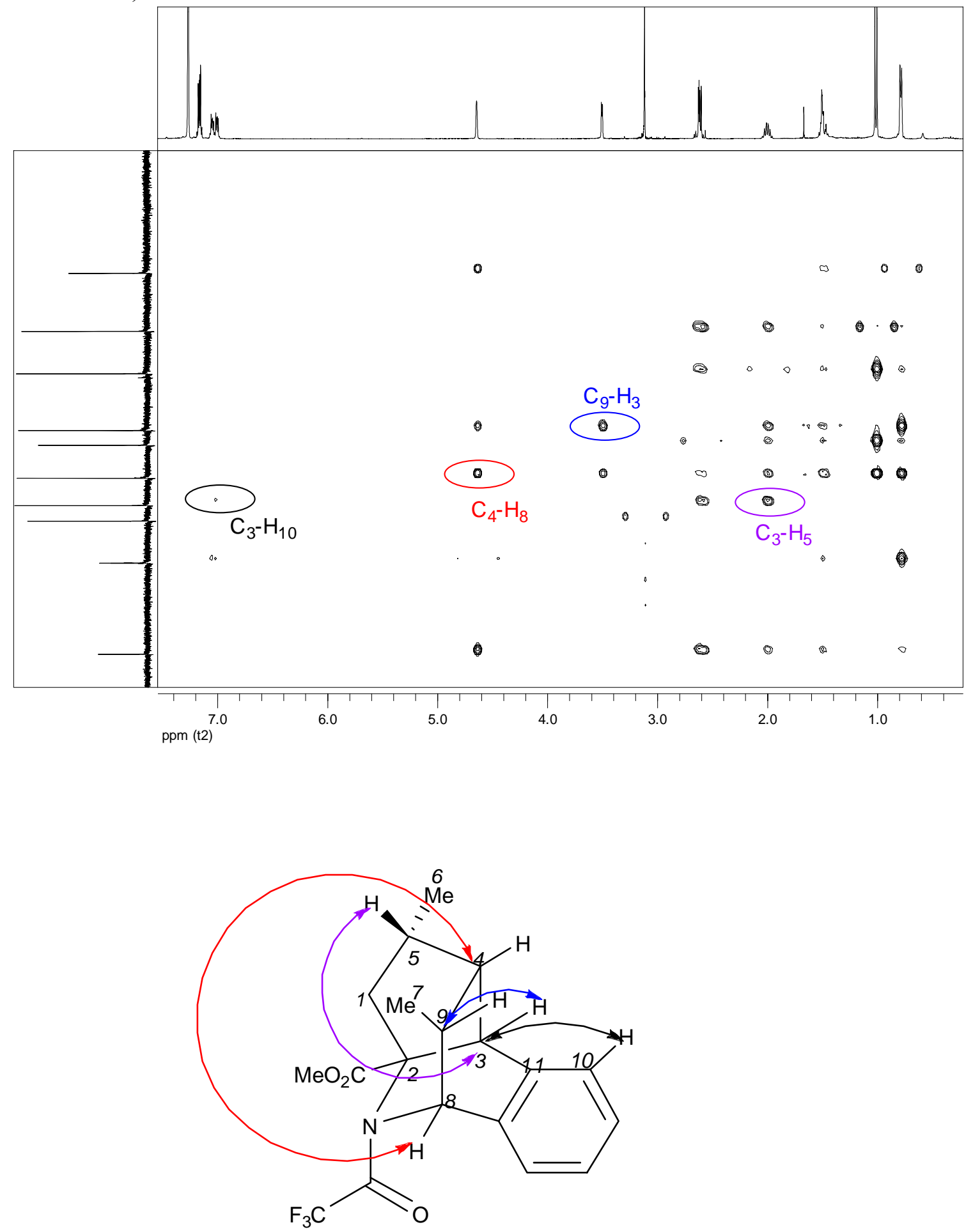

$\mathrm{C}_{3}-\mathrm{H}_{10}$ crosspeak provides evidence for the formation of $\mathrm{C}_{3}-C_{11}$ bond 
Expanded NOESY Spectrum of 36 (in benzene-d6)
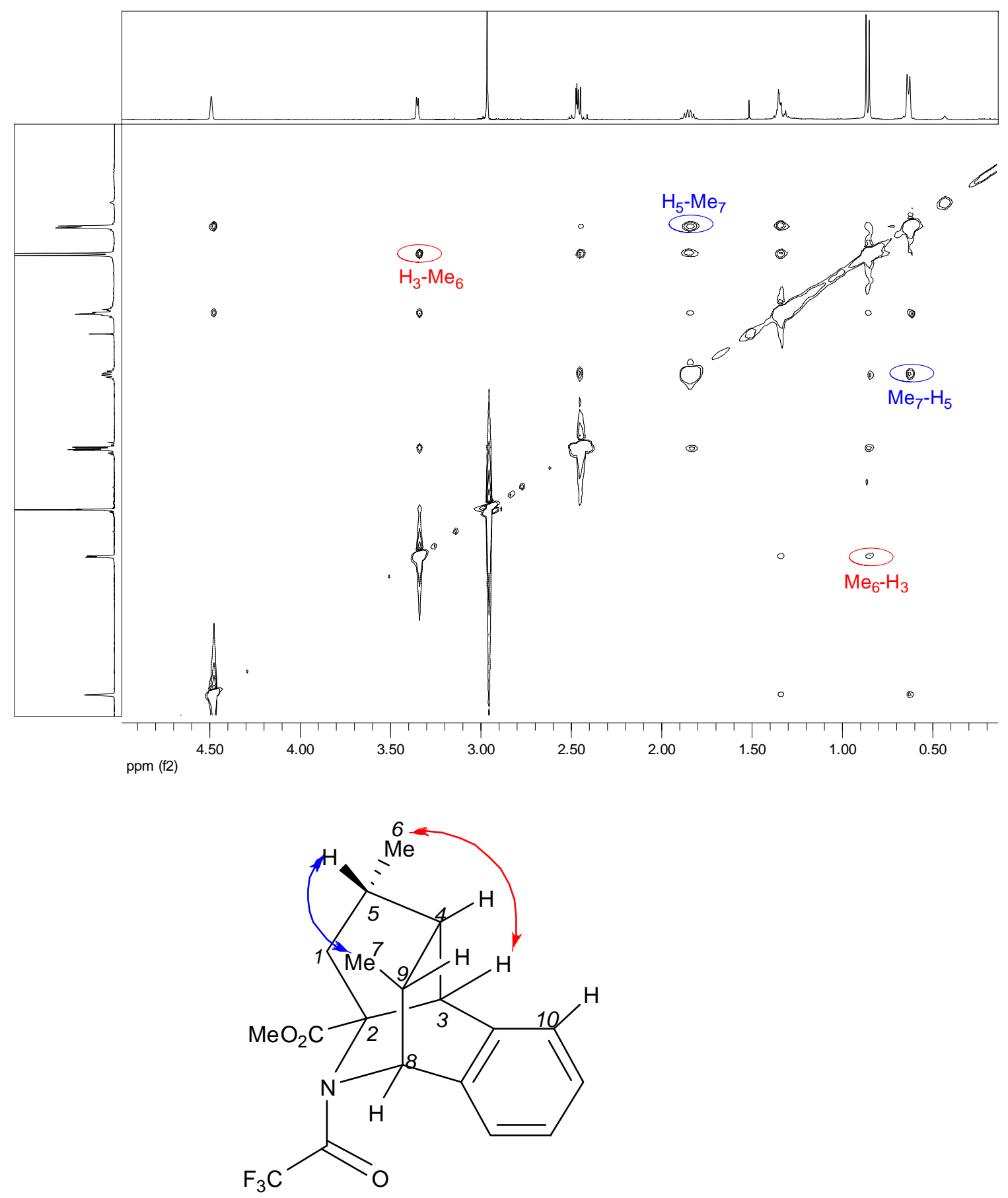

The indicated crosspeaks conf irm the cis relationship between $\mathrm{Me}_{6}$ and $\mathrm{H}_{3}$ and $\mathrm{Me}_{7}$ and $\mathrm{H}_{5}$ 


\section{${ }^{1} \mathrm{H}$ NMR Spectrum of 37.}
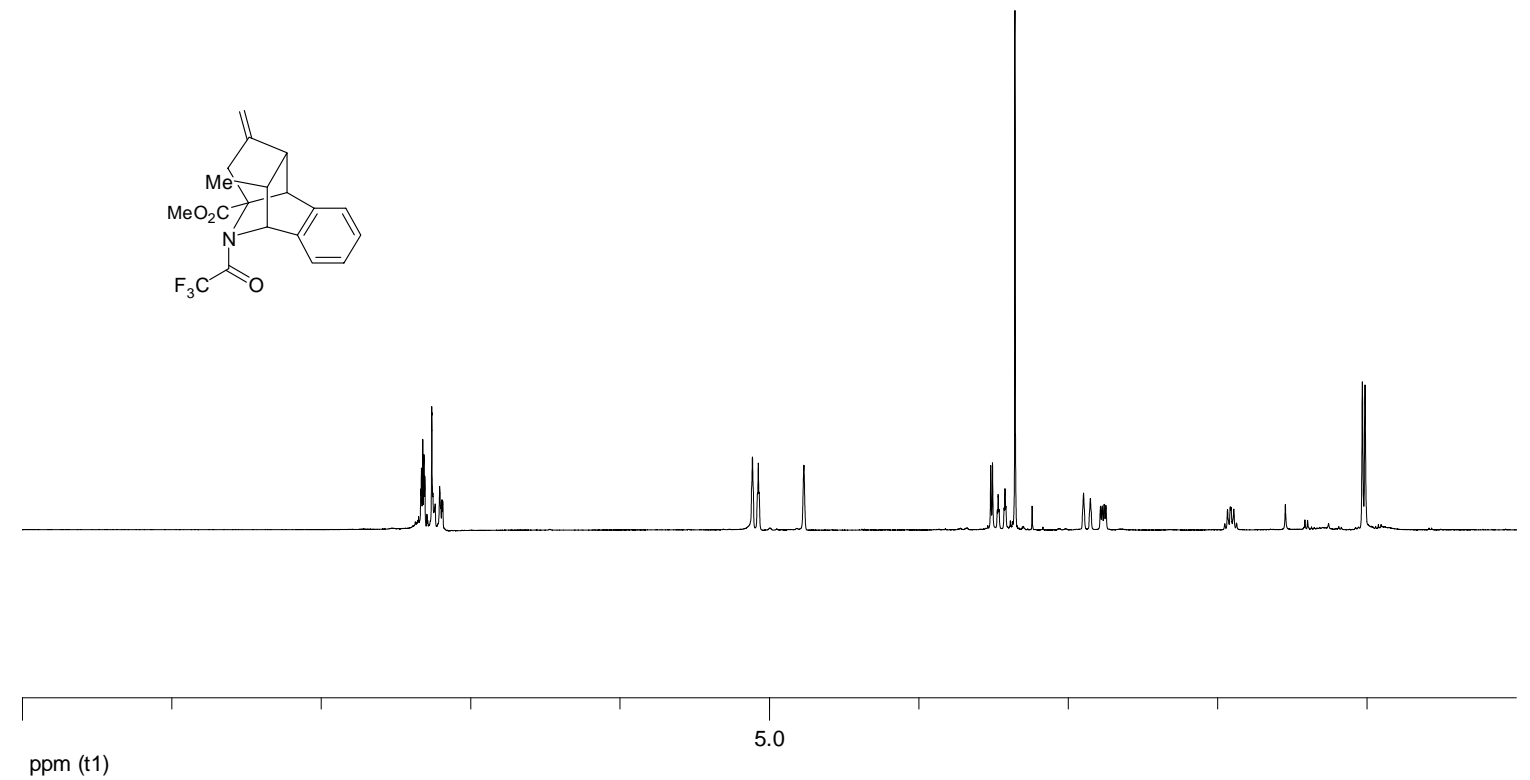

${ }^{13}$ C NMR Spectrum of 37.

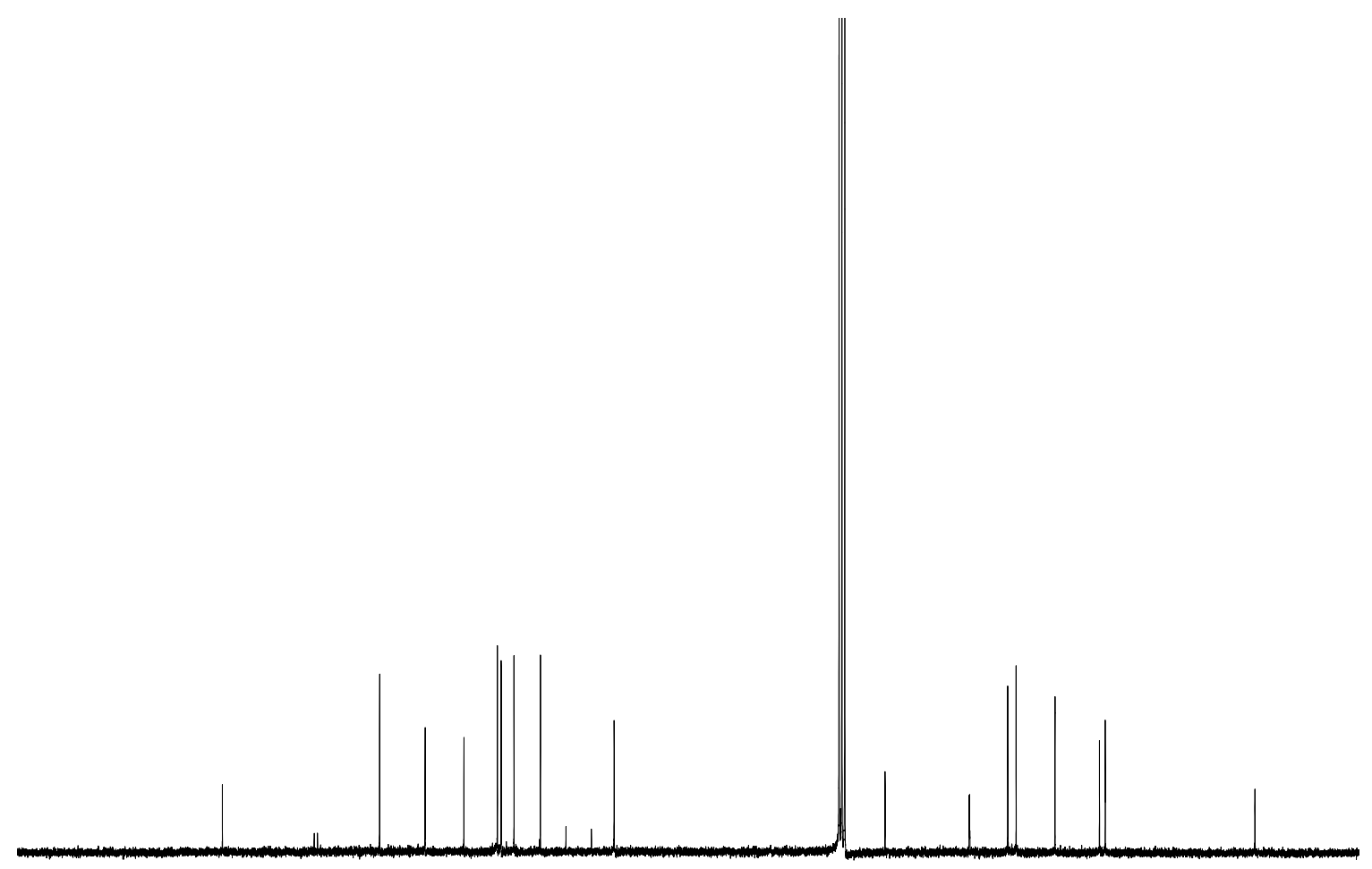

SI-31 


\section{Hydrogenation}

Compound 38: A solution of compound $37(12 \mathrm{mg}, 0.03 \mathrm{mmol})$ and $\mathrm{PtO}_{2}(0.61 \mathrm{mg}$,

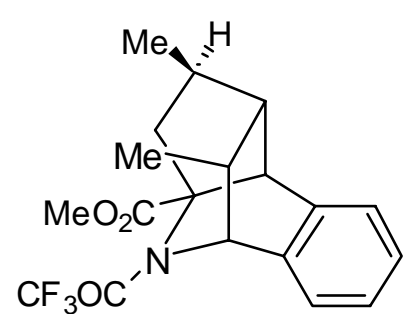
$0.003 \mathrm{mmol}, 0.10$ equiv) in ethylacetate $(2 \mathrm{~mL})$ was evacuated and filled with a balloon of hydrogen. After stirring at room temperature for $5 \mathrm{~min}$, the crude reaction mixture was filtered through celite and concentrated. Silica gel chromatography with ethyl acetate in hexane (from $0 \%$ to $30 \%$ ) gave the desired product 38 (10 $\mathrm{mg}, 84 \%$ yield) as a viscous oil. ${ }^{1} \mathrm{H}$ NMR (400 $\left.\mathrm{MHz}, \mathrm{CD}_{3} \mathrm{CN}\right) \delta$ 7.34-7.25 (m, 3H), 7.25-7.20 (m, 1H), $4.78(\mathrm{~b}$ $\mathrm{s}, 1 \mathrm{H}), 3.38(\mathrm{~d}, J=3.9 \mathrm{~Hz}, 1 \mathrm{H}), 3.34(\mathrm{~s}, 3 \mathrm{H}), 3.17(\mathrm{dd}, J=14.3,10.9 \mathrm{~Hz}, 1 \mathrm{H}), 2.72-2.59$ $(\mathrm{m}, 1 \mathrm{H}), 2.26-2.20(\mathrm{~m}, 1 \mathrm{H}), 2.07-1.99(\mathrm{~m}, 1 \mathrm{H}), 1.68(\mathrm{dd}, J=14.4,10.0 \mathrm{~Hz}, 1 \mathrm{H}), 1.29(\mathrm{~d}$, $J=5.61 \mathrm{~Hz}, 3 \mathrm{H}), 1.27(\mathrm{~d}, J=5.63 \mathrm{~Hz}, 3 \mathrm{H}) .{ }^{13} \mathrm{C} \mathrm{NMR}\left(100 \mathrm{MHz}, \mathrm{CD}_{3} \mathrm{CN}\right) \delta 170.6$, $155.4(\mathrm{q}, J=35.6 \mathrm{~Hz}), 140.4,135.8,128.8,128.2,126.8,122.6,117.5(\mathrm{q}, J=282.2 \mathrm{~Hz})$, 71.6, $59.3(\mathrm{~d}, J=3.0 \mathrm{~Hz}), 54.3,52.6,43.0,42.2,40.4,38.3,17.5,17.0$. HRMS (CI/NH3) $\mathrm{m} / \mathrm{z}$ calculated for $\left[\mathrm{C}_{20} \mathrm{H}_{20} \mathrm{NO}_{3} \mathrm{~F}_{3}+\mathrm{Na}\right]$ 390.1293, found 390.1286. $v_{\max } 2962,1740,1684$, $1203,1143 \mathrm{~cm}^{-1} \cdot[\alpha]_{\mathrm{D}}{ }^{20}+17.9^{\circ}\left(\mathrm{c}=1.0, \mathrm{CHCl}_{3}\right)$. 
7. NMR Spectra of Hydrogenated Product 38.

${ }^{1} \mathbf{H}$ NMR spectrum of 38 (in acetonitrile-d3).
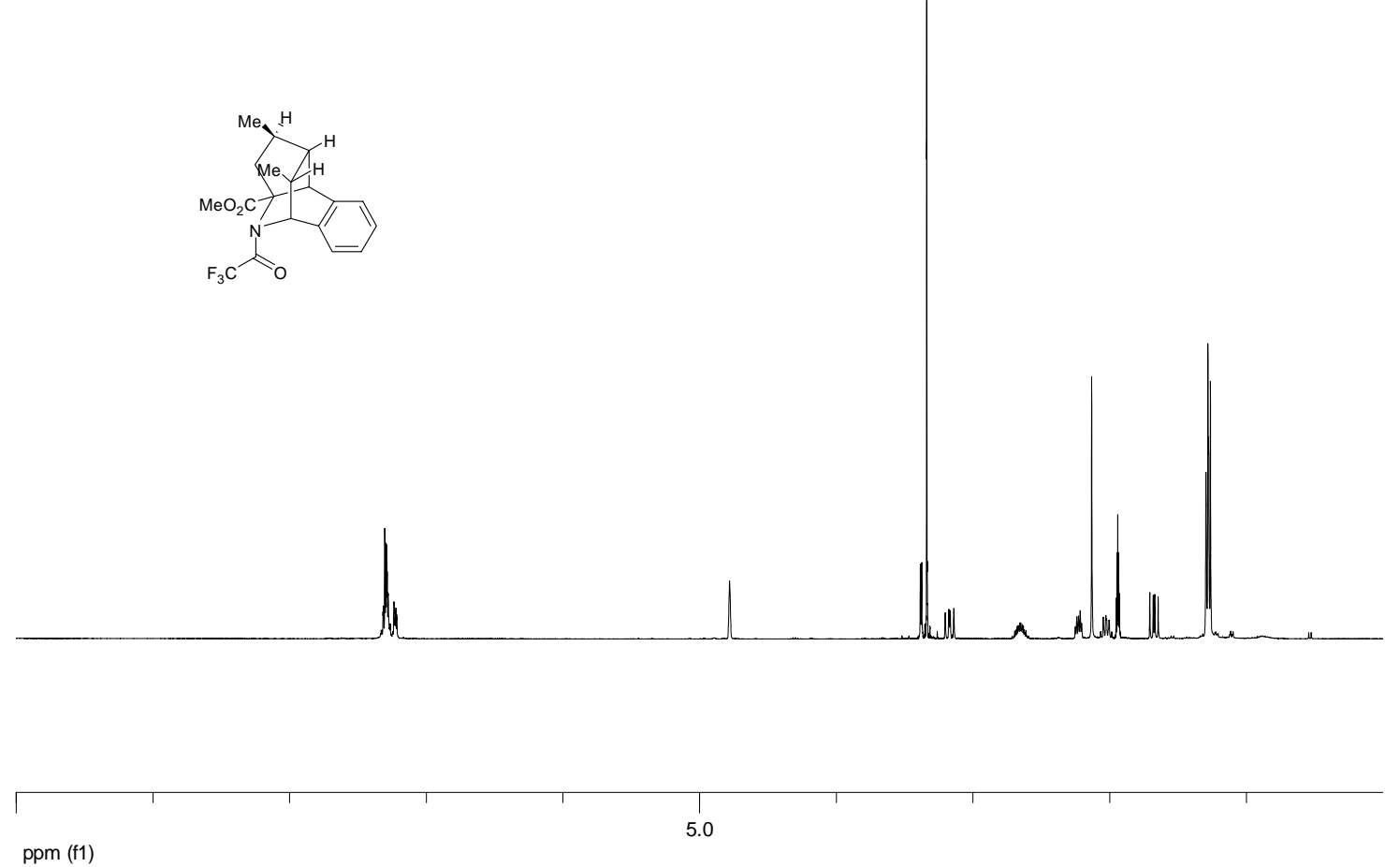

${ }^{13}$ C NMR Spectrum of 38. (in acetonitrile-d3)

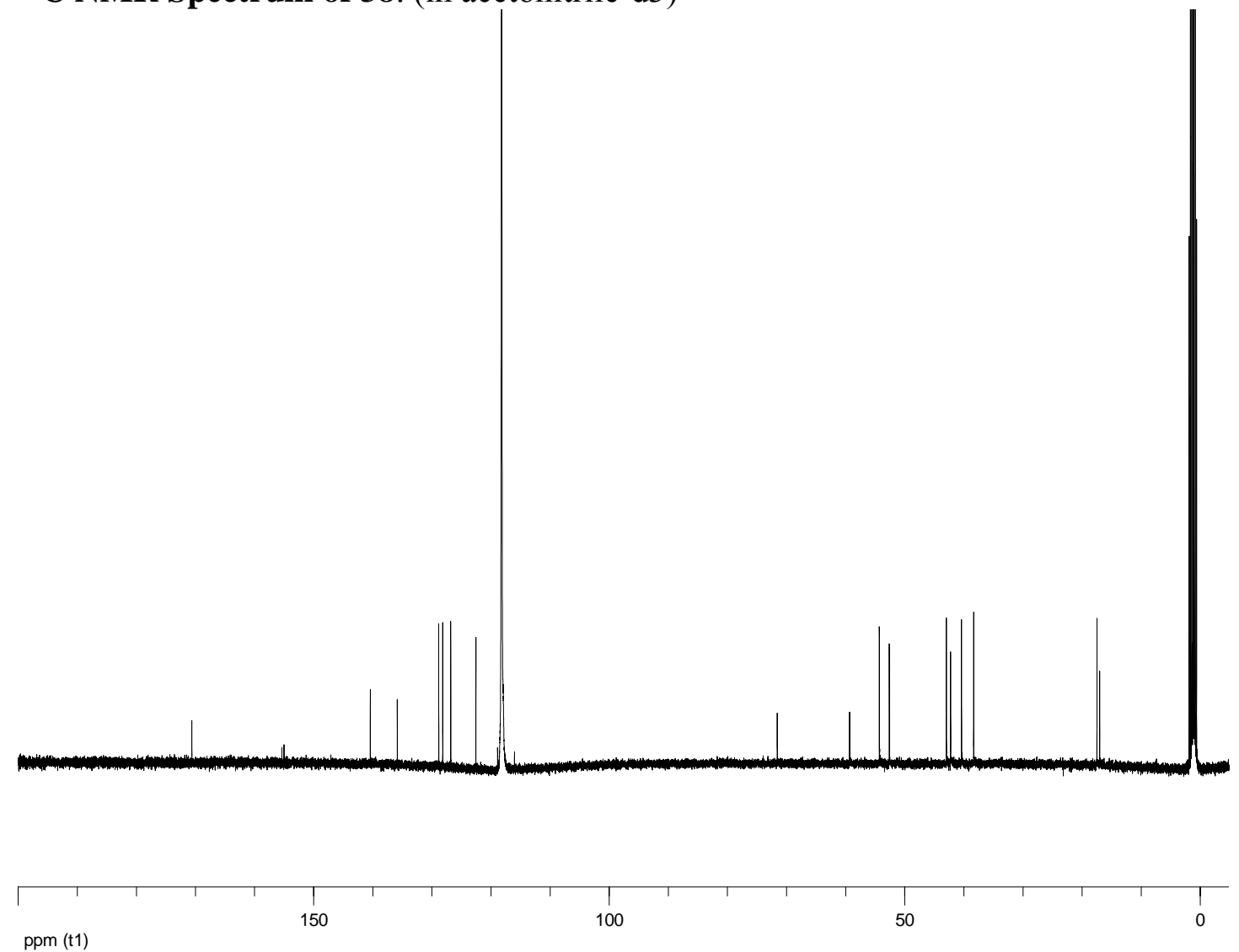


Expanded COSY Spectrum of 38. (in acetonitrile-d3)
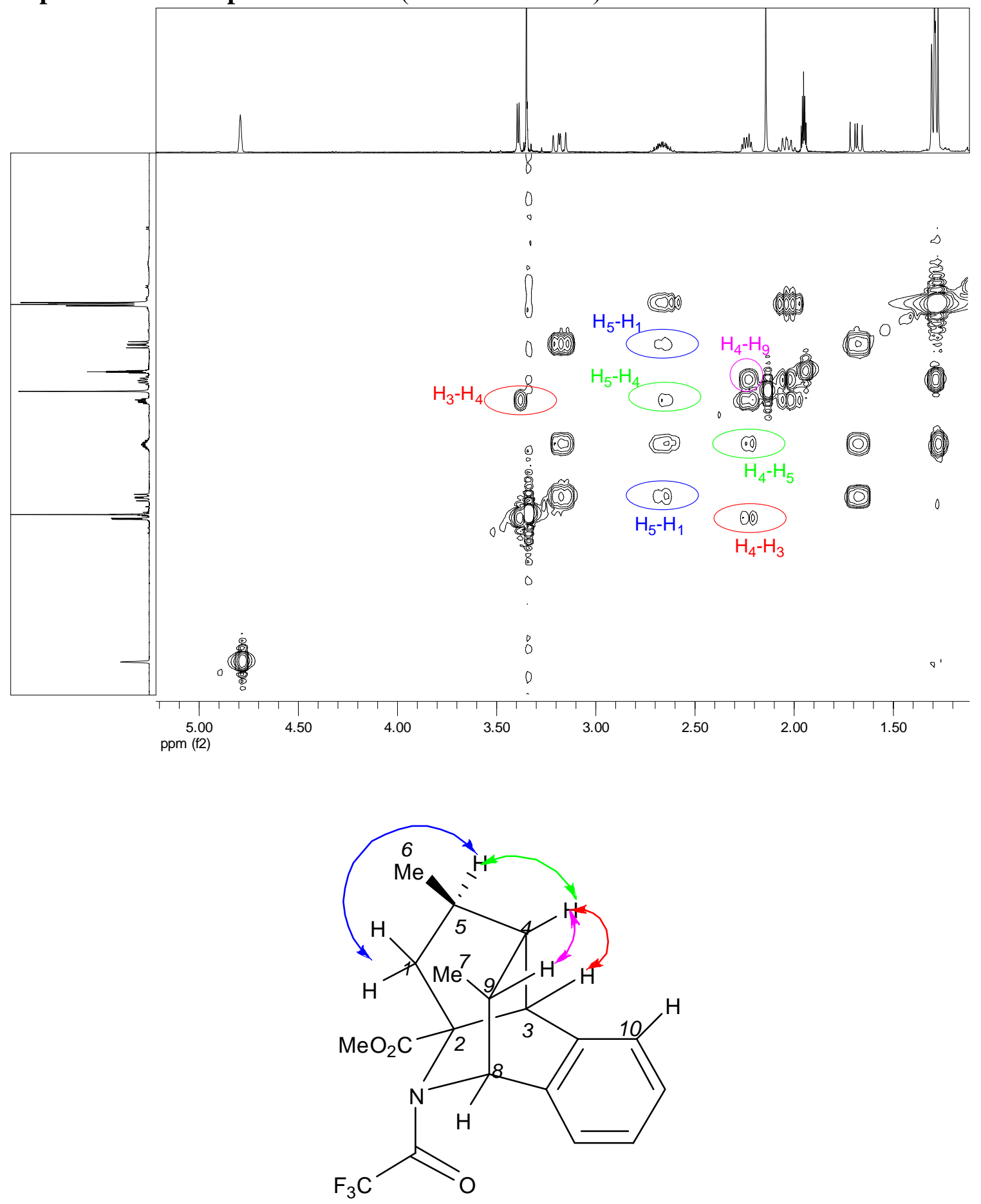
Expanded 1D NOE Spectrum of 38 indicating cis relationship between $\mathbf{H}_{\mathbf{5}}$ and $\mathrm{H}_{\mathbf{3}}$ : (in acetonitrile-d3) (multiplet corresponding to $\mathrm{H}_{5}$ is irradiated)
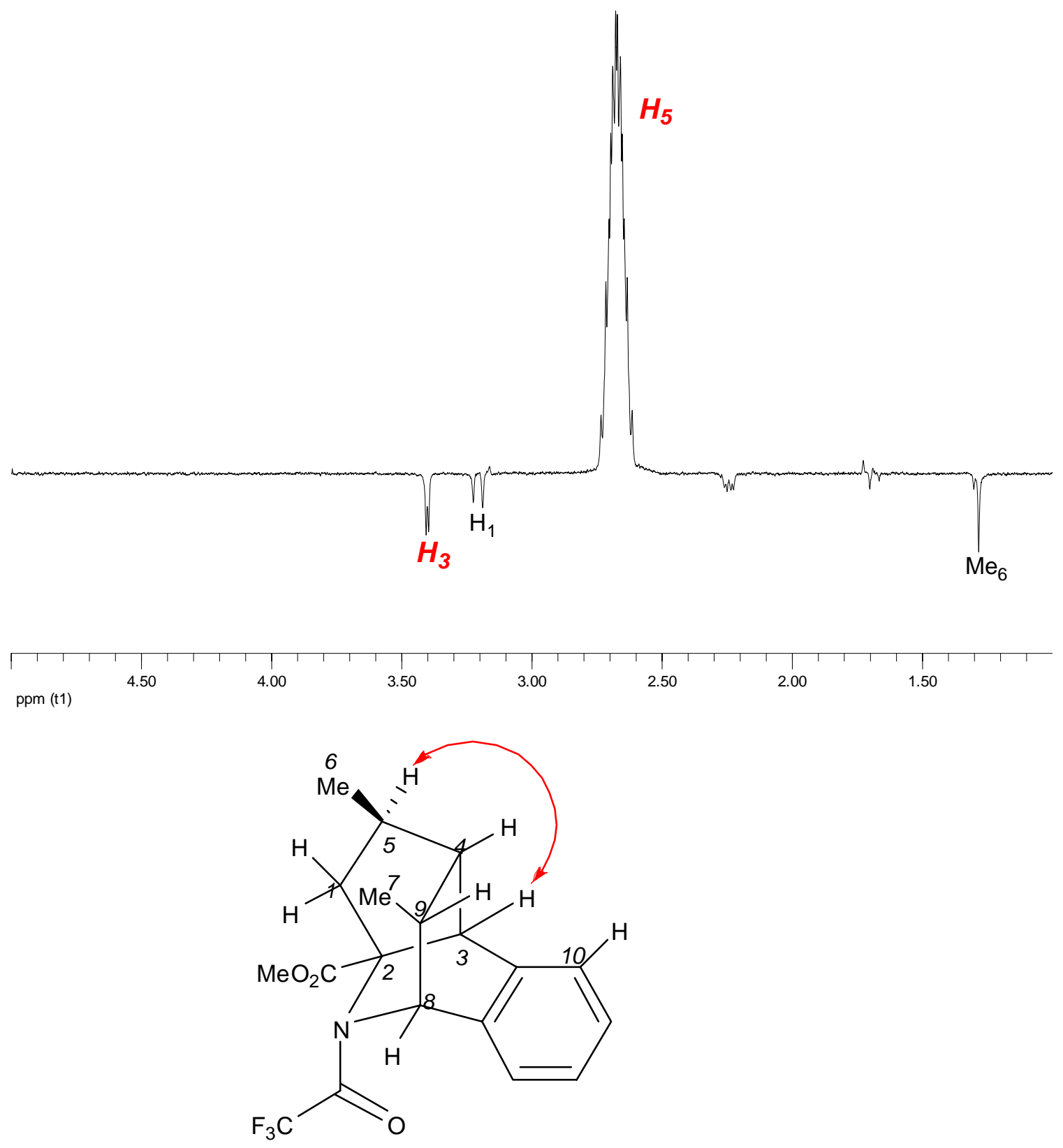